ACT-09/00, CTP-TAMU-15/00

TPI-MINN-00/34,UMN-TH-1912-00

OUTP-00-28P

hep-ph/0006331

May 2000

\title{
Left-Right Symmetric Heterotic-String Derived Models
}

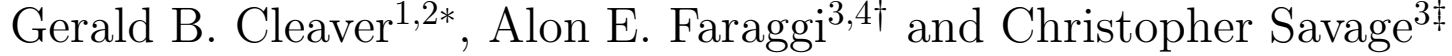

1 Center for Theoretical Physics, Texas A\&M University, College Station, TX 77843

2 Astro Particle Physics Group, Houston Advanced Research Center (HARC), The Mitchell Campus, Woodlands, TX 77381

3 Department of Physics, University of Minnesota, Minneapolis, MN 55455

4 Theoretical Physics Department, University of Oxford, Oxford, OX1 3NP

\begin{abstract}
Recently it was demonstrated that free fermionic heterotic-strings can produce models with solely the Minimal Supersymmetric Standard Model states in the low energy spectrum. This unprecedented result provides further strong evidence for the possibility that the true string vacuum shares some of the properties of the free fermionic models. Past free fermionic models have focused on several possible unbroken observable $S O(10)$ subgroups at the string scale, which include the flipped $S U(5)$ (FSU5), the Pati-Salam (PS) string models, and the string Standard-like Models (SLM). We extend this study to include the case in which the $S O(10)$ symmetry is broken to the Left-Right Symmetric (LRS) gauge group, $S O(10) \rightarrow S U(3)_{C} \times U(1)_{B-L} \times S U(2)_{L} \times S U(2)_{R}$. We present several models of this type and discuss their phenomenological features. The most striking new outcome of the LRS string models, in contrast to the case of the FSU5, the PS, and the SLM string models, is that they can produce effective field theories that are free of Abelian anomalies. We discuss the distinction between the two types of free fermionic models which result in the presence, or absence, of an anomalous $U(1)$. As a counter example we also present a LRS model that does contain an anomalous $U(1)$. Additionally, we discuss how in string models the Standard Model spectrum may arise from the three 16 representations of $S O(10)$, while the weak-hypercharge does not have the canonical $S O(10)$ embedding.
\end{abstract}

*gcleaver@rainbow.physics.tamu.edu

†faraggi@mnhepw.hep.umn.edu

‡csavage@physics.spa.umn.edu 


\section{Introduction}

Recently it was demonstrated that free fermionic heterotic string models can produce models with solely the spectrum of the Minimal Supersymmetric Standard Model (MSSM) in the effective four dimensional field theory [1]. This achievement provides further motivation to improve our understanding of this particular class of heterotic string models. The realistic free fermionic models consist of a large number of three generation models which differ in their detailed phenomenological characteristics. All these models share an underlying $\mathbb{Z}_{2} \times \mathbb{Z}_{2}$ orbifold structure which arises from a basic set of boundary condition basis vectors, the so-called NAHE-set?. With this fundamental set incorporated as a necessary ingredient in the construction, one then finds that three generation models, with the canonical $S O(10)$ embedding of the Standard Model spectrum naturally arise. Furthermore, one of the generic features of semi-realistic string constructions is the existence of numerous massless states beyond the MSSM spectrum, some of which carry fractional electric charge and hence must decouple from the low energy spectrum. Recently, and for the first time since the advent of string phenomenology [4], we have been able to demonstrate in the FNY free fermionic model [5], that free fermionic models can also produce models with solely the MSSM states in the light spectrum. We will refer to such a heterotic string model, as a Minimal Standard Heterotic String Model (MSHSM)用.

At the level of the NAHE set, denoted by $\left\{\mathbf{1}, \mathbf{S}, \mathbf{b}_{1}, \mathbf{b}_{2}, \mathbf{b}_{3}\right\}$, the gauge group is $S O(10) \times S O(6)^{3} \times E_{8}$. The $S O(6)^{3}$ symmetries are horizontal flavor symmetries; the $E_{8}$ factor gives rise to the hidden gauge group at this stage and the Standard Model universal gauge group arises from the $S O(10)$ factor. Beyond the NAHE set the construction of the realistic free fermionic models proceeds by adding three or four additional boundary condition basis vectors. These additional basis vectors fix the final $S O(10)$ subgroup in the effective field theory, and at the same time reduce the number of generations to three, one from each of the sectors $\mathbf{b}_{1}, \mathbf{b}_{2}$ and $\mathbf{b}_{3}$. The models studied to date have focused on three possibilities for the final $S O(10)$ subgroup: the flipped SU(5) (FSU5) with $S O(10) \rightarrow S U(5) \times U(1)$ [2]; the PatiSalam (PS) type models with $S O(10) \rightarrow S O(6) \times S O(4)$ [6]; and the standard-like models $(\mathrm{SLM})$ with $S O(10) \rightarrow S U(3) \times S U(2) \times U(1)^{2}$ [7].

\footnotetext{
${ }^{*}$ NAHE $=$ pretty in Hebrew. The NAHE set was first employed by Nanopoulos, Antoniadis, Hagelin and Ellis in the construction of the flipped $S U(5)$ heterotic-string model [2]. Its vital role in the realistic free fermionic models has been emphasized in ref. [3].

$\dagger$ It is interesting to note that among the perturbative heterotic-string orbifold models the free fermionic models are the only ones which have yielded three generations with the canonical $S O(10)$ embedding

¥ It should be emphasized that the success of the FNY model in producing a MSHSM should not be regarded as indicating that the FNY model represents the correct string vacuum. Indeed, much further elaborate studies would be needed to support such a claim. The phenomenological success of the free fermionic models implies that the generic structure afforded by the NAHE set is favorable for obtaining agreement with the phenomenological characteristics suggested by the Standard Model data.
} 
In this paper we extend the analysis of the three generation free fermionic models to models with the left-right symmetric (LRS) gauge group, i.e. with $S O(10) \rightarrow$ $S U(3) \times U(1) \times S U(2)_{L} \times S U(2)_{R}$. Our primary motivation is to extend our understanding of the general properties of the realistic free fermionic models. It should also be noted, however, that LRS models have been extensively studied as attractive field theoretic extensions of the Standard Model, in which parity violation is understood to arise from the spontaneous breakdown of $S U(2)_{R}$. Further phenomenological advantages of LRS models include its potential role in providing a solution to the strong CP problem and to the SUSY CP problem [8]. From a supersymmetric grand unification perspective the LRS symmetric models have the appealing property that $\mathrm{R}$-parity appears as a gauged symmetry. From a string unification perspective the LRS models, similar to the PS and SLM string models, have the advantage that they can incorporate the stringy doublet-triplet splitting mechanism [17]. In contrast to the MSSM, and similar to the PS models, the LRS models produce Yukawa couplings of the up and down quark families to a Higgs bi-doublet, which present the danger of inducing Flavor Changing Neutral Currents (FCNC) at an unacceptable rate [9].

One of the interesting aspects of the LRS string models that we show is the possible absence of an anomalous $U(1)$ symmetry. As is well known, generically string models with $(2,0)$ world-sheet supersymmetry give rise to an anomalous $U(1)$ symmetry 110, 11, 12. In this paper we present the first examples of semi-realistic $(2,0)$ heterotic string models in which all the $U(1)$ symmetries are anomaly free.

Our paper is organized as follows. In Section 2 we give a quick review of the field theory content of the LRS models that we aim to construct. In Section 3 we discuss the symmetry breaking pattern in the string models. In Section 1 we present the first example of a LRS string model. The full massless spectrum and related quantum numbers with respect to the four dimensional gauge group are determined, as well as all superpotential terms up to quintic order. In Section 5 we offer a variation of the LRS model in which the $U(1)_{B-L}$ symmetry is enhanced to a non-Abelian symmetry. The full massless spectrum with quantum numbers and the superpotential are derived for this model as well. In Section 6 we discuss the absence of an anomalous $U(1)$ symmetry in our first two examples of LRS string models. As a counter example we also present a LRS model which does possess an anomalous $U(1)$. Section 7 contains a phenomenological discussion and our conclusions.

\section{The supersymmetric left-right symmetric model}

In this section we briefly summarize the field theory structure of the type of models that we aim to construct from string theory in this paper. The observable sector gauge symmetry we seek is $S U(3)_{C} \times S U(2)_{L} \times S U(2)_{R} \times U(1)_{B-L}$. Such models are reminiscent of the PS type string models, but differ from them by the fact that the $S U(4)$ gauge group is broken to $S U(3) \times U(1)_{B-L}$ already at the string level. Similar to the PS models, the LRS models possess the $S O(10)$ embedding. The 
quarks and leptons are accommodated in the following representations:

$$
\begin{aligned}
Q_{L}^{i} & =(3,2,1)_{\frac{1}{6}}=\left(\begin{array}{l}
u \\
d
\end{array}\right)^{i} \\
Q_{R}^{i} & =(\overline{3}, 1,2)_{-\frac{1}{6}}=\left(\begin{array}{l}
d^{c} \\
u^{c}
\end{array}\right)^{i} \\
L_{L}^{i} & =(1,2,1)_{-\frac{1}{2}}=\left(\begin{array}{l}
\nu \\
e
\end{array}\right)^{i} \\
L_{R}^{i} & =(1,1,2)_{\frac{1}{2}}=\left(\begin{array}{l}
e^{c} \\
\nu^{c}
\end{array}\right)^{i} \\
h & =(1,2,2)_{0}=\left(\begin{array}{ll}
h_{+}^{u} & h_{0}^{d} \\
h_{0}^{u} & h_{-}^{d}
\end{array}\right)
\end{aligned}
$$

where $h^{d}$ and $h^{u}$ are the two low energy supersymmetric superfields associated with the Minimal Supersymmetric Standard Model. The breaking of $S U(2)_{R}$ could be achieved with the VEV of $h$. However, this will result with too light $W_{R}^{ \pm}$gauge boson masses. Additional fields that can be used to break $S U(2)_{R}$ must therefore be postulated. The simplest set would consist of two fields $H+\bar{H}$ transforming as $(1,1,2)_{-\frac{1}{2}}+(1,1, \overline{2})_{\frac{1}{2}}$. When $H$ and $\bar{H}$ acquire VEVs along their neutral components $S U(2)_{R} \times U(1)_{B-L}$ is broken to the Standard Model weak-hypercharge, $U(1)_{Y}$. With this symmetry breaking pattern the bi-doublet Higgs field may split into the two Higgs doublet $h^{u}$ and $h^{d}$ of the MSSM.

The LRS string models can also contain Higgs fields that transform as $(3,1,1)$ and $(\overline{3}, 1,1)$, which originate from the vectorial 10 representation of $S O(10)$. These color triplets mediate proton decay through dimension five operators, and consequently must be sufficiently heavy to insure agreement with the proton lifetime. An important advantage of the LRS breaking pattern, with $S O(10) \rightarrow S O(6) \times S O(4)$ at the string construction level, is that these color triplets may be projected out by the GSO projections, and therefore need not be present in the low energy spectrum. In the PS models, however, the Higgs representations that induce $S U(4) \times S U(2)_{R} \rightarrow$ $S U(3)_{C} \times U(1)_{Y}$ contain Higgs triplet representations. In the supersymmetric PS models the color triplets in the vectorial representation $(6,1,1)$ are used to give large mass to the Higgs color triplets, by the superpotential terms $\lambda_{2} H H D+\lambda_{3} \bar{H} \bar{H} \bar{D}$, when the fields $H$ and $\bar{H}$ develop a large VEV of the order of the GUT scale. Therefore, the stringy doublet-triplet splitting mechanism is useful only in models with $S U(3)_{C} \times$ $S U(2)_{L} \times U(1)^{2}$, or $S U(3)_{C} \times S U(2)_{L} \times S U(2)_{R} \times U(1)_{B-L}$, as the $S O(10)$ subgroup which remains unbroken by the GSO projections.

The LRS models should also contain four additional singlet fields $\phi_{0}$ and $\phi_{i=1,2,3}$. $\phi_{0}$ acquires a VEV of the order of the electroweak scale which induces the electroweak Higgs doublet mixing, while $\phi_{i}$ are used to construct an extended see-saw mechanism which generate left-right Majorana masses for the left-handed neutrinos. The tree 
level superpotential of the model is given by:

$$
W=\lambda_{i j}^{1} Q_{L}^{i} Q_{R}^{j} h+\lambda_{i j}^{2} L_{L}^{i} L_{R}^{j} h+\lambda_{i j}^{3} L_{R}^{i} \bar{H} \phi^{j}+\lambda^{4} h h \phi^{0}+\lambda_{5} \Phi^{3}
$$

where $\Phi=\left\{\phi^{i}, \phi^{0}\right\}$. The superpotential in eq. (2.6) leads to the neutrino mass matrix

$$
\left(\begin{array}{ccc}
0 & m_{u}^{i j} & 0 \\
m_{u}^{j i} & 0 & \langle\bar{H}\rangle \\
0 & \langle\bar{H}\rangle & \left\langle\phi_{0}\right\rangle
\end{array}\right)
$$

whose diagonalization gives three light neutrinos with masses of the order $\left\langle\phi_{0}\right\rangle\left(m_{u}^{i j} /\langle\bar{H}\rangle\right)^{2}$ and gives heavy mass, of order $\langle\bar{H}\rangle$, to the right-handed neutrinos.

Below the scale of $S U(2)_{R}$ breaking the left-right symmetric models should reproduce the spectrum and couplings of the MSSM. As our interest here is primarily in the string construction of left-right symmetric models we do not enter into the field theory details, which have been amply studied in the literature [ 8$]$. There is one important issue, however, that deserves mention. As seen from eq. (2.6) in the left-right symmetric models both up-quark and down-quark masses arise from the coupling to the Higgs bi-doublet. This introduces the danger of inducing Flavor Changing Neutral Currents (FCNC) at an unacceptable rate. A possible solution is to use two bi-doublet Higgs representations, one of which is used to give masses to the up-type quarks, while the second is used to give masses to the down-type quarks. This, however, introduces a bi-doublet splitting problem. Namely, we must insure that one Higgs multiplet remains light to give mass to the up- or down-type quarks, while the second Higgs multiplet in the respective bi-doublet becomes sufficiently heavy so as to avoid problems with FCNC. Arguably, this can be achieved in a field theory setting. However, the bi-doublet splitting mechanisms that have been discussed in the literature [13] utilize $S U(2)$ triplet representations that are, in general, not present in the free fermionic string models. Therefore, whether or not bi-doublet splitting can be achieved in the left-right symmetric string models is an open question, which we will not address in this paper.

We emphasize that our intent here is not to construct a fully realistic leftright symmetric model, but merely to study the structure of free fermionic string models with this choice of the $S O(10)$ subgroup. In this respect we note that the bi-doublet splitting problem introduces further motivation for the choice of $S U(3) \times S U(2) \times U(1)^{2}$ as the $S O(10)$ subgroup which remains unbroken after application of the string GSO projections. Thus, while the doublet-triplet splitting problem does not distinguish between the PS string model $(S O(10) \rightarrow S O(6) \times S O(4))$, or LRS string model $\left(S O(10) \rightarrow S U(3) \times S U(2)^{2} \times U(1)\right)$, and the SLM string model $\left(S O(10) \rightarrow S U(3) \times S U(2) \times U(1)^{2}\right)$, the bi-doublet splitting problem favors the later choice. The SLM string models provide a stringy solution both to the doublettriplet splitting problem, as well as the bi-doublet splitting problem. In this respect it should also be noted that the choice of $S U(4)_{C} \times S U(2)_{L} \times U(1)_{T_{3_{R}}}$ as the unbroken $S O(10)$ subgroup also achieves these two tasks. Study of this case is left for future work. 


\section{Left-right symmetric free fermionic models}

A model in the free fermionic formulation [14] is constructed by choosing a consistent set of boundary condition basis vectors. The basis vectors, $\mathbf{b}_{k}$, span a finite additive group $\Xi=\sum_{k} n_{k} \mathbf{b}_{k}$ where $n_{k}=0, \cdots, N_{z_{k}}-1$. The physical massless states in the Hilbert space of a given sector $\alpha \in \Xi$, are obtained by acting on the vacuum with bosonic and fermionic operators and by applying the generalized GSO projections. The $U(1)$ charges, $Q(f)$, for the unbroken Cartan generators of the four dimensional gauge group are in one to one correspondence with the $U(1)$ currents $f^{*} f$ for each complex fermion $\mathrm{f}$, and are given by:

$$
Q(f)=\frac{1}{2} \alpha(f)+F(f)
$$

where $\alpha(f)$ is the boundary condition of the world-sheet fermion $f$ in the sector $\alpha$, and $F_{\alpha}(f)$ is a fermion number operator counting each mode of $f$ once (and if $f$ is complex, $f^{*}$ minus once). For periodic fermions, $\alpha(f)=1$, the vacuum is a spinor representation of the Clifford algebra of the corresponding zero modes. For each periodic complex fermion $f$ there are two degenerate vacua $|+\rangle,|-\rangle$, annihilated by the zero modes $f_{0}$ and $f_{0}{ }^{*}$ and with fermion numbers $F(f)=0,-1$, respectively.

The realistic models in the free fermionic formulation are generated by a basis of boundary condition vectors for all world-sheet fermions [2, 5, 6, 7, 15, 16]. The basis is constructed in two stages. The first stage consists of the NAHE set [3, 7], which is a set of five boundary condition basis vectors, $\left\{\mathbf{1}, \mathbf{S}, \mathbf{b}_{1}, \mathbf{b}_{2}, \mathbf{b}_{3}\right\}$. The gauge group after the NAHE set is $S O(10) \times S O(6)^{3} \times E_{8}$ with $N=1$ space-time supersymmetry. The vector $\mathbf{S}$ is the supersymmetry generator and the superpartners of the states from a given sector $\alpha$ are obtained from the sector $\mathbf{S}+\alpha$. The space-time vector bosons that generate the gauge group arise from the Neveu-Schwarz (NS) sector and from the sector $\zeta \equiv \mathbf{1}+\mathbf{b}_{1}+\mathbf{b}_{2}+\mathbf{b}_{3}$. The NS sector produces the generators of $S O(10) \times S O(6)^{3} \times S O(16)$. The sector $\zeta$ produces the spinorial 128 of $S O(16)$ and completes the hidden gauge group to $E_{8}$. The vectors $\mathbf{b}_{1}, \mathbf{b}_{2}$ and $\mathbf{b}_{3}$ produce 48 spinorial 16's of $S O(10)$, sixteen from each sector $\mathbf{b}_{1}, \mathbf{b}_{2}$ and $\mathbf{b}_{3}$. The vacuum of these sectors contains eight periodic worldsheet fermions, five of which produce the charges under the $S O(10)$ group, while the remaining three periodic fermions generate charges with respect to the flavor symmetries. Each of the sectors $\mathbf{b}_{1}, \mathbf{b}_{2}$ and $\mathbf{b}_{3}$ is charged with respect to a different set of flavor quantum numbers, $S O(6)_{1,2,3}$.

The NAHE set divides the 44 right-moving and 20 left-moving real internal fermions in the following way: $\bar{\psi}^{1, \cdots, 5}$ are complex and produce the observable $S O(10)$ symmetry; $\bar{\phi}^{1, \cdots, 8}$ are complex and produce the hidden $E_{8}$ gauge group; $\left\{\bar{\eta}^{1}, \bar{y}^{3, \cdots, 6}\right\}$, $\left\{\bar{\eta}^{2}, \bar{y}^{1,2}, \bar{\omega}^{5,6}\right\},\left\{\bar{\eta}^{3}, \bar{\omega}^{1, \cdots, 4}\right\}$ give rise to the three horizontal $S O(6)$ symmetries. The left-moving $\{y, \omega\}$ states are also divided into the sets $\left\{y^{3, \cdots, 6}\right\},\left\{y^{1,2}, \omega^{5,6}\right\},\left\{\omega^{1, \cdots, 4}\right\}$. The left-moving $\chi^{12}, \chi^{34}, \chi^{56}$ states carry the supersymmetry charges. Each sector $\mathbf{b}_{1}$, $\mathbf{b}_{2}$ and $\mathbf{b}_{3}$ carries periodic boundary conditions under $\left(\psi^{\mu} \mid \bar{\psi}^{1, \cdots, 5}\right)$ and one of the three groups: $\left(\chi_{12},\left\{y^{3, \cdots, 6} \mid \bar{y}^{3, \cdots 6}\right\}, \bar{\eta}^{1}\right),\left(\chi_{34},\left\{y^{1,2}, \omega^{5,6} \mid \bar{y}^{1,2} \bar{\omega}^{5,6}\right\}, \bar{\eta}^{2}\right),\left(\chi_{56},\left\{\omega^{1, \cdots, 4} \mid \bar{\omega}^{1, \cdots 4}\right\}, \bar{\eta}^{3}\right)$. 
The second stage of the basis construction consist of adding three additional basis vectors to the NAHE set. Three additional vectors are needed to reduce the number of generations to three, one from each sector $\mathbf{b}_{1}, \mathbf{b}_{2}$ and $\mathbf{b}_{3}$. One specific example is given in Table (4.1). The choice of boundary conditions to the set of real internal fermions $\{y, \omega \mid \bar{y}, \bar{\omega}\}^{1, \cdots, 6}$ determines the low energy properties, such as the number of generations, Higgs doublet-triplet splitting and Yukawa couplings.

The $S O(10)$ gauge group is broken to one of its subgroups $S U(5) \times U(1), S O(6) \times$ $S O(4)$ or $S U(3) \times S U(2) \times U(1)^{2}$ by the assignment of boundary conditions to the set $\bar{\psi}_{\frac{1}{2}}^{1 \cdots 5}$ :
1. $b\left\{\bar{\psi}_{\frac{1}{2}}^{1 \cdots 5}\right\}=\left\{\frac{1}{2} \frac{1}{2} \frac{1}{2} \frac{1}{2} \frac{1}{2}\right\} \Rightarrow S U(5) \times U(1)$,
2. $b\left\{\bar{\psi}_{\frac{1}{2}}^{1 \cdots 5}\right\}=\{11100\} \Rightarrow S O(6) \times S O(4)$.

To break the $S O(10)$ symmetry to $S U(3)_{C} \times S U(2)_{L} \times U(1)_{C} \times U(1)_{L}$ 円 both steps, 1 and 2, are used, in two separate basis vectors. Similarly, the breaking pattern $S O(10) \rightarrow S U(3)_{C} \times S U(2)_{L} \times S U(2)_{R} \times U(1)_{B-L}$ is achieved by the following assignment in two separate basis vectors

1. $b\left\{\bar{\psi}_{\frac{1}{2}}^{1 \cdots 5}\right\}=\{11100\} \Rightarrow S O(6) \times S O(4)$,

2. $b\left\{\bar{\psi}_{\frac{1}{2}}^{\frac{1}{2} \cdots 5}\right\}=\left\{\frac{1}{2} \frac{1}{2} \frac{1}{2} 00\right\} \Rightarrow S U(3)_{C} \times U(1)_{C} \times S U(2)_{L} \times S U(2)_{R}$.

We comment here that a recurring feature of some of the three generation free fermionic heterotic string models is the emergence of a combination of the basis vectors which extend the NAHE set,

$$
\mathbf{X}=n_{\alpha} \alpha+n_{\beta} \beta+n_{\gamma} \gamma
$$

for which $\mathbf{X}_{L} \cdot \mathbf{X}_{L}=0$ and $\mathbf{X}_{R} \cdot \mathbf{X}_{R} \neq 0$. Such a combination may produce additional space-time vector bosons, depending on the choice of GSO phases. These additional space-time vector bosons enhance the four dimensional gauge group. This situation is similar to the presence of the combination of the NAHE set basis vectors $\mathbf{1}+$ $\mathbf{b}_{1}+\mathbf{b}_{2}+\mathbf{b}_{3}$, which enhances the hidden gauge group, at the level of the NAHE set, from $S O(16)$ to $E_{8}$. As we discuss below, we often find, although not always, that either the $S U(3)_{C}$ or the $U(1)_{C}$ symmetry is enhanced to $S U(4)_{C}$ or $S U(2)_{C}$, respectively. Therefore, we will present models with and without gauge symmetry enhancement. In the free fermionic models this type of gauge symmetry enhancement in the observable sector is, in general, family universal and is intimately related to the $\mathbb{Z}_{2} \times \mathbb{Z}_{2}$ orbifold structure which underlies the realistic free fermionic models. Such enhanced symmetries were shown to forbid proton decay mediating operators to all orders of nonrenormalizable terms [16].

\footnotetext{
${ }^{*} U(1)_{C}=\frac{3}{2} U(1)_{B-L} ; U(1)_{L}=2 U(1)_{T_{3_{R}}}$.
} 


\section{Left-right symmetric models without enhanced symmetry}

As our first example of a left-right symmetric free fermionic heterotic string model we consider Model 1, specified below. The boundary conditions of the three basis vectors which extend the NAHE set are shown in Table (4.1). Also given in Table (4.1) are the pairings of left- and right-moving real fermions from the set $\{y, \omega \mid \bar{y}, \bar{\omega}\}$. These fermions are paired to form either complex, left- or right-moving, fermions, or Ising model operators, which combine a real left-moving fermion with a real rightmoving fermion. The generalized GSO coefficients determining the physical massless states of Model 1 appear in matrix (4.2).

LRS Model 1 Boundary Conditions:

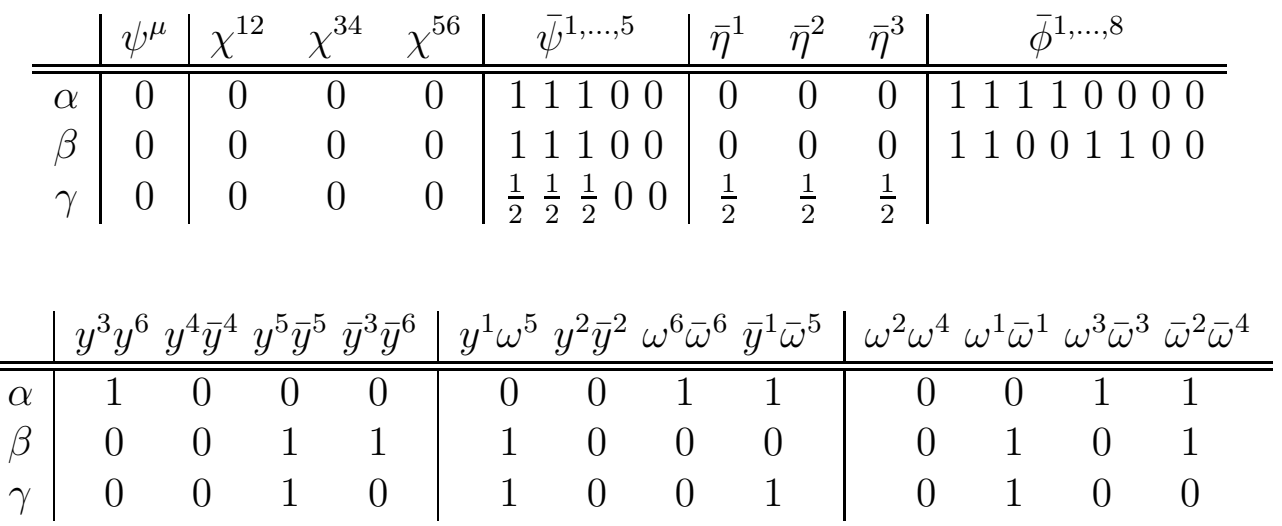

LRS Model 1 Generalized GSO Coefficients:

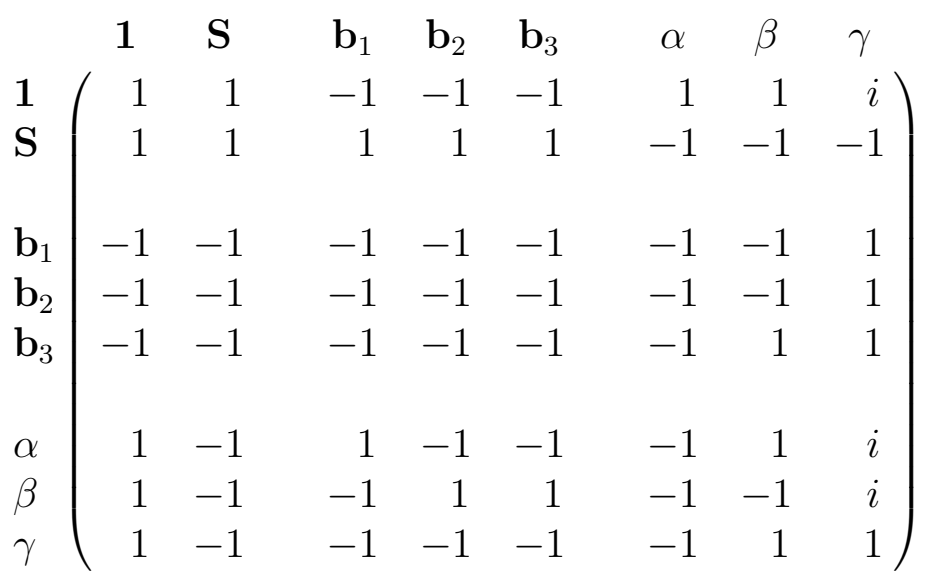

In matrix (4.2) only the entries above the diagonal are independent and those below and on the diagonal are fixed by the modular invariance constraints. Blank lines are inserted to emphasize the division of the free phases between the different sectors of the realistic free fermionic models. Thus, the first two lines involve only the GSO phases of $c\left(\begin{array}{c}\{\mathbf{1}, \mathbf{S}\} \\ \mathbf{a}_{i}\end{array}\right)$. The set $\{\mathbf{1}, \mathbf{S}\}$ generates the $N=4$ model with $\mathbf{S}$ being the 
space-time supersymmetry generator and therefore the phases $c\left(\begin{array}{c}\mathbf{s} \\ \mathbf{a}_{i}\end{array}\right)$ are those that control the space-time supersymmetry in the superstring models. Similarly, in the free fermionic models, sectors with periodic and anti-periodic boundary conditions, of the form of $\mathbf{b}_{i}$, produce the chiral generations. The phases $c\left(\begin{array}{l}\mathbf{b}_{i} \\ \mathbf{b}_{j}\end{array}\right)$ determine the chirality of the states from these sectors.

In the free fermionic models the basis vectors $\mathbf{b}_{i}$ are those that respect the $S O(10)$ symmetry while the vectors denoted by Greek letters are those that break the $S O(10)$ symmetry. As the Standard Model matter states arise from sectors which preserve the $S O(10)$ symmetry, the phases that fix the Standard Model charges are, in general, the phases $c\left(\begin{array}{c}\mathbf{b}_{i} \\ \mathbf{a}_{i}\end{array}\right)$. On the other hand, the basis vectors of the form $\{\alpha, \beta, \gamma\}$ break the $S O(10)$ symmetry. The phases associated with these basis vectors are associated with exotic physics, beyond the Standard Model. These phases, therefore, also affect the final four dimensional gauge symmetry.

The final gauge group in Model 1 arises as follows: In the observable sector the NS boundary conditions produce gauge group generators for

$$
S U(3)_{C} \times S U(2)_{L} \times S U(2)_{R} \times U(1)_{C} \times U(1)_{1,2,3} \times U(1)_{4,5,6}
$$

Thus, the $S O(10)$ symmetry is broken to $S U(3) \times S U(2)_{L} \times S U(2)_{R} \times U(1)_{C}$, as discussed above, where,

$$
U(1)_{C}=\operatorname{Tr} U(3)_{C} \Rightarrow Q_{C}=\sum_{i=1}^{3} Q\left(\bar{\psi}^{i}\right) .
$$

The flavor $S O(6)^{3}$ symmetries are broken to $U(1)^{3+n}$ with $(n=0, \cdots, 6)$. The first three, denoted by $U(1)_{j}(j=1,2,3)$, arise from the world-sheet currents $\bar{\eta}^{j} \bar{\eta}^{j *}$. These three $U(1)$ symmetries are present in all the three generation free fermionic models which use the NAHE set. Additional horizontal $U(1)$ symmetries, denoted by $U(1)_{j}$ $(j=4,5, \ldots)$, arise by pairing two real fermions from the sets $\left\{\bar{y}^{3, \cdots, 6}\right\},\left\{\bar{y}^{1,2}, \bar{\omega}^{5,6}\right\}$, and $\left\{\bar{\omega}^{1, \cdots, 4}\right\}$. The final observable gauge group depends on the number of such pairings. In this model there are the pairings, $\bar{y}^{3} \bar{y}^{6}, \bar{y}^{1} \bar{\omega}^{5}$ and $\bar{\omega}^{2} \bar{\omega}^{4}$, which generate three additional $U(1)$ symmetries, denoted by $U(1)_{4,5,6}$.

In the hidden sector the NS boundary conditions produce the generators of

$$
S U(2)_{1} \times U(1)_{H_{1}} \times S U(2)_{2} \times U(1)_{H_{2}} \times U(1)_{7,8,9,10}
$$

where $S U(2)_{1}$ and $S U(2)_{2}$ arise from the complex world-sheet fermions $\left\{\bar{\phi}^{3}, \bar{\phi}^{4}\right\}$ and $\left\{\bar{\phi}^{5}, \bar{\phi}^{6}\right\}$, respectively; and $U(1)_{H_{1}}$ and $U(1)_{H_{2}}$ correspond to the combinations of

\footnotetext{
${ }^{\dagger}$ It is important to note that the existence of these three additional $U(1)$ currents is correlated with a superstringy doublet-triplet splitting mechanism [17]. Due to these extra $U(1)$ symmetries the color triplets from the NS sector are projected out of the spectrum by the GSO projections while the electroweak doublets remain in the light spectrum.
} 
world-sheet charges

$$
\begin{aligned}
Q_{H_{1}} & =Q\left(\bar{\phi}^{1}\right)-Q\left(\bar{\phi}^{2}\right)+\sum_{i=5}^{7} Q(\bar{\phi})^{i}-Q(\bar{\phi})^{8} \\
Q_{H_{2}} & =\sum_{i=1}^{4} Q(\bar{\phi})^{i}-Q\left(\bar{\phi}^{7}\right)-Q(\bar{\phi})^{8} .
\end{aligned}
$$

The charges under the remaining four orthogonal $U(1)$ combinations are given by

$$
\begin{aligned}
Q_{7} & =Q\left(\bar{\phi}^{1}\right)+Q\left(\bar{\phi}^{8}\right), \\
Q_{8} & =Q\left(\bar{\phi}^{2}\right)+Q\left(\bar{\phi}^{7}\right), \\
Q_{9} & =Q\left(\bar{\phi}^{1}\right)-Q\left(\bar{\phi}^{3}\right)-Q\left(\bar{\phi}^{4}\right)-Q\left(\bar{\phi}^{5}\right)-Q\left(\bar{\phi}^{6}\right)-Q\left(\bar{\phi}^{8}\right), \\
Q_{10} & =Q\left(\bar{\phi}^{2}\right)-Q\left(\bar{\phi}^{3}\right)-Q\left(\bar{\phi}^{4}\right)+Q\left(\bar{\phi}^{5}\right)+Q\left(\bar{\phi}^{6}\right)-Q\left(\bar{\phi}^{7}\right) .
\end{aligned}
$$

The sector $\zeta \equiv 1+\mathbf{b}_{1}+\mathbf{b}_{2}+\mathbf{b}_{3}$ produces the representations $(2,1)_{ \pm 4,0}$ and $(1,2)_{0, \pm 4}$ of $S U(2)_{H_{1}} \times U(1)_{H_{1}}$ and $S U(2)_{H_{2}} \times U(1)_{H_{2}}$, raising the symmetry to $S U(3)_{H_{1}} \times S U(3)_{H_{2}}$. Thus, the hidden $E_{8}$ symmetry is broken to $S U(3)_{H_{1}} \times S U(3)_{H_{2}} \times U(1)_{7,8,9,10}$.

In addition to the graviton, dilaton, antisymmetric sector and spin-1 gauge bosons, the NS sector gives two pairs of electroweak doublets, transforming as $(1,2,2,0)$ under $S U(3)_{C} \times S U(2)_{L} \times S U(2)_{R} \times U(1)_{C}$; three pairs of $S O(10)$ singlets with $U(1)_{1,2,3}$ charges; and three singlets of the entire four dimensional gauge group. The states from the sectors $\mathbf{b}_{j}(j=1,2,3)$ produce the three light generations. These states and their decomposition under the entire gauge group are shown in Table 1 in Appendix A. The remaining massless states and their quantum numbers also appear in Table 1.

\subsection{Model 1 Superpotential}

We now turn to the superpotential of the model. The cubic level and higher order terms in the superpotential are obtained by calculating the correlators between the vertex operators. The non-vanishing terms must be invariant under all the symmetries of the string models and must satisfy all the string selection rules [18]. The full superpotential has been analyzed up to order $N=6$. Below we give the cubic and quartic order terms and the quintic order terms are given in Appendix B. We divide the superpotential terms into four sets. Terms in the first set contain the states that transform nontrivialy under the Standard Model gauge group. Terms in the second set contain only states that are singlets of all non-Abelian groups. Terms in the third set contain states that transform nontrivialy under the unbroken hidden $E_{8}$ non-Abelian subgroup, while terms in the fourth set contain both Standard Model and Hidden Sector states. We indicate when no terms of a given type are found at a specific order. 
$W_{3}$ (observable):

$$
\begin{array}{rlllll}
h_{1} h_{2} \Phi_{3} & +h_{1} Q_{L_{1}} Q_{R_{1}} & +h_{1} L_{L_{1}} L_{R_{1}} & +h_{1} \mathcal{L}_{L_{23}}^{+} \mathcal{L}_{R_{23}}^{-} & +h_{1} \mathcal{L}_{L_{23}}^{-} \mathcal{L}_{R_{23}}^{+} & +h_{1} \mathcal{L}_{L_{1}}^{+} \mathcal{L}_{R_{1}}^{-} \\
+h_{1} \mathcal{L}_{L_{1}}^{-} \mathcal{L}_{R_{1}}^{+} & +h_{2} Q_{L_{2}} Q_{R_{2}} & +h_{2} L_{L_{2}} L_{R_{2}} & +h_{2} \mathcal{L}_{L_{13}}^{+} \mathcal{L}_{R_{13}}^{-} & +h_{2} \mathcal{L}_{L_{13}}^{-} \mathcal{L}_{R_{13}}^{+} & +h_{2} \mathcal{L}_{L_{2}}^{+} \mathcal{L}_{R_{2}}^{-} \\
+h_{2} \mathcal{L}_{L_{2}}^{-} \mathcal{L}_{R_{2}}^{+} & +Q_{L_{3}} \bar{D}_{\alpha \beta} \mathcal{L}_{L_{3}}^{-} & +Q_{R_{3}} D_{\alpha \beta} \mathcal{L}_{R_{3}}^{+} & +L_{L_{1}} \mathcal{L}_{L_{1}}^{+} \bar{\phi}_{5} & +L_{L_{2}} \mathcal{L}_{L_{2}}^{+} \bar{\phi}_{6} & +L_{L_{3}} \mathcal{L}_{L_{3}}^{+} \bar{\phi}_{7} \\
+L_{R_{1}} \mathcal{L}_{R_{1}}^{-} \phi_{5} & +L_{R_{2}} \mathcal{L}_{R_{2}}^{-} \phi_{6} & +L_{R_{3}} \mathcal{L}_{R_{3}}^{-} \phi_{7} & +\mathcal{L}_{L_{13}}^{-} \mathcal{L}_{L_{2}}^{-} \bar{\phi}_{9} & +\mathcal{L}_{L_{23}}^{+} \mathcal{L}_{L_{1}}^{-} \bar{\phi}_{8} & +\mathcal{L}_{R_{13}}^{+} \mathcal{L}_{R_{2}}^{+} \phi_{9} \\
+\mathcal{L}_{R_{23}}^{-} \mathcal{L}_{R_{1}}^{+} \phi_{8} & & & & &
\end{array}
$$

$W_{3}($ singlets $)$ :

$$
\begin{array}{clllll}
\Phi_{3} \phi_{1} \bar{\phi}_{1} & +\Phi_{3} \phi_{2} \bar{\phi}_{2} & +\Phi_{3} \phi_{3} \bar{\phi}_{3} & +\Phi_{3} \phi_{4} \bar{\phi}_{4} & +\Phi_{3} \phi_{10} \bar{\phi}_{10} & +\Phi_{3} \phi_{11} \bar{\phi}_{11} \\
+\Phi_{12} \bar{\Phi}_{13} \Phi_{23} & +\Phi_{12} \phi_{1} \phi_{4} & +\Phi_{12} \phi_{2} \phi_{3} & +\Phi_{12} \phi_{6} \bar{\phi}_{5} & +\bar{\Phi}_{12} \Phi_{13} \bar{\Phi}_{23} & +\bar{\Phi}_{12} \bar{\phi}_{1} \bar{\phi}_{4} \\
+\bar{\Phi}_{12} \bar{\phi}_{2} \bar{\phi}_{3} & +\bar{\Phi}_{12} \bar{\phi}_{6} \phi_{5} & +\Phi_{13} \phi_{7} \bar{\phi}_{5} & +\bar{\Phi}_{13} \bar{\phi}_{7} \phi_{5} & +\Phi_{23} \phi_{7} \bar{\phi}_{6} & +\bar{\Phi}_{23} \bar{\phi}_{7} \phi_{6}
\end{array}
$$

\begin{tabular}{|c|c|}
\hline $\begin{aligned} & Q_{L_{1}} Q_{L_{3}} Q_{R_{1}} Q_{R_{3}} \\
+ & Q_{L_{1}} Q_{R_{1}} \mathcal{L}_{L_{3}}^{-} \mathcal{L}_{R_{3}}^{+} \\
+ & Q_{L_{2}} Q_{R_{1}} \mathcal{L}_{L_{23}}^{+} \mathcal{L}_{R_{13}}^{-} \\
+ & Q_{L_{2}} Q_{R_{2}} \mathcal{L}_{L_{3}}^{+} \mathcal{L}_{R_{3}}^{-} \\
+ & Q_{L_{3}} Q_{R_{3}} L_{L_{1}} L_{R_{1}} \\
+ & Q_{L_{3}} Q_{R_{3}} \mathcal{L}_{L_{23}}^{-} \mathcal{L}_{R_{23}}^{+} \\
+ & L_{L_{1}} L_{L_{3}} L_{R_{1}} L_{R_{3}} \\
+ & L_{L_{1}} \mathcal{L}_{L_{23}}^{-} \phi_{1} \phi_{11} \\
+ & L_{L_{2}} L_{R_{2}} \mathcal{L}_{L_{3}}^{+} \mathcal{L}_{R_{3}}^{-} \\
+ & L_{L_{3}} L_{R_{3}} \mathcal{L}_{L_{13}}^{-} \mathcal{L}_{R_{13}}^{+} \\
+ & L_{L_{3}} L_{R_{3}} \mathcal{L}_{L_{2}}^{+} \mathcal{L}_{R_{2}}^{-} \\
+ & L_{R_{2}} \mathcal{L}_{R_{13}}^{-} \phi_{2} \bar{\phi}_{11} \\
+ & \mathcal{L}_{L_{12}}^{+} \mathcal{L}_{L_{1}}^{+} \mathcal{L}_{R_{12}}^{-} \mathcal{L}_{R_{1}}^{-} \\
+ & \mathcal{L}_{L_{12}}^{-} \mathcal{L}_{L_{13}}^{+} \mathcal{L}_{R_{12}}^{+} \mathcal{L}_{R_{13}}^{-} \\
+ & \mathcal{L}_{L_{12}}^{-} \mathcal{L}_{L_{1}}^{+} \mathcal{L}_{R_{23}}^{+} \mathcal{L}_{R_{3}}^{-} \\
+ & \mathcal{L}_{L_{13}}^{+} \mathcal{L}_{L_{3}}^{+} \mathcal{L}_{R_{12}}^{-} \mathcal{L}_{R_{2}}^{-} \\
+ & \mathcal{L}_{L_{13}}^{-} \mathcal{L}_{L_{3}}^{-} \mathcal{L}_{R_{13}}^{+} \mathcal{L}_{R_{3}}^{+} \\
+ & \mathcal{L}_{L_{23}}^{-} \mathcal{L}_{L_{3}}^{+} \mathcal{L}_{R_{12}}^{+} \mathcal{L}_{R_{1}}^{-} \\
+ & \mathcal{L}_{L_{1}}^{-} \mathcal{L}_{L_{3}}^{+} \mathcal{L}_{R_{1}}^{+} \mathcal{L}_{R_{3}}^{-} \\
+ & \mathcal{L}_{L_{2}}^{-} \mathcal{L}_{L_{3}}^{-} \mathcal{L}_{R_{2}}^{+} \mathcal{L}_{R_{3}}^{+}\end{aligned}$ & $\begin{array}{l}+Q_{L_{1}} Q_{R_{1}} L_{L_{3}} L_{R_{3}} \\
+Q_{L_{1}} Q_{R_{2}} \mathcal{L}_{L_{13}}^{+} \mathcal{L}_{R_{23}}^{-} \\
+Q_{L_{2}} Q_{R_{1}} \mathcal{L}_{L_{23}}^{-} \mathcal{L}_{R_{13}}^{+} \\
+Q_{L_{2}} Q_{R_{2}} \mathcal{L}_{L_{3}}^{-} \mathcal{L}_{R_{3}}^{+} \\
+Q_{L_{3}} Q_{R_{3}} L_{L_{2}} L_{R_{2}} \\
+Q_{L_{3}} Q_{R_{3}} \mathcal{L}_{L_{1}}^{+} \mathcal{L}_{R_{1}}^{-} \\
+L_{L_{1}} L_{R_{1}} \mathcal{L}_{L_{12}}^{+} \mathcal{L}_{R_{12}}^{-} \\
+L_{L_{1}} \mathcal{L}_{L_{23}}^{-} \phi_{2} \phi_{10} \\
+L_{L_{2}} L_{R_{2}} \mathcal{L}_{L_{3}}^{-} \mathcal{L}_{R_{3}}^{+} \\
+L_{L_{3}} L_{R_{3}} \mathcal{L}_{L_{23}}^{+} \mathcal{L}_{R_{23}}^{-} \\
+L_{L_{3}} L_{R_{3}} \mathcal{L}_{L_{2}}^{-} \mathcal{L}_{R_{2}}^{+} \\
+\mathcal{L}_{L_{12}}^{+} \mathcal{L}_{L_{13}}^{+} \mathcal{L}_{R_{12}}^{-} \mathcal{L}_{R_{13}}^{-} \\
+\mathcal{L}_{L_{12}}^{+} \mathcal{L}_{L_{1}}^{-} \mathcal{L}_{R_{12}}^{-} \mathcal{L}_{R_{1}}^{+} \\
+\mathcal{L}_{L_{12}}^{-} \mathcal{L}_{L_{13}}^{-} \mathcal{L}_{R_{12}}^{+} \mathcal{L}_{R_{13}}^{+} \\
+\mathcal{L}_{L_{12}}^{-} \mathcal{L}_{L_{1}}^{-} \mathcal{L}_{R_{12}}^{+} \mathcal{L}_{R_{1}}^{+} \\
+\mathcal{L}_{L_{13}}^{+} \mathcal{L}_{L_{3}}^{+} \mathcal{L}_{R_{13}}^{-} \mathcal{L}_{R_{3}}^{-} \\
+\mathcal{L}_{L_{23}}^{+} \mathcal{L}_{L_{2}}^{+} \mathcal{L}_{R_{13}}^{-} \mathcal{L}_{R_{1}}^{-} \\
+\mathcal{L}_{L_{23}}^{-} \mathcal{L}_{L_{3}}^{+} \mathcal{L}_{R_{23}}^{+} \mathcal{L}_{R_{3}}^{-} \\
+\mathcal{L}_{L_{1}}^{-} \mathcal{L}_{L_{3}}^{-} \mathcal{L}_{R_{1}}^{+} \mathcal{L}_{R_{3}}^{+}\end{array}$ \\
\hline
\end{tabular}

$W_{3}$ (hidden):

$$
\Phi_{3} H_{1} \bar{H}_{1} \quad+\Phi_{3} H_{2} \bar{H}_{2}
$$

$W_{4}$ (observable):

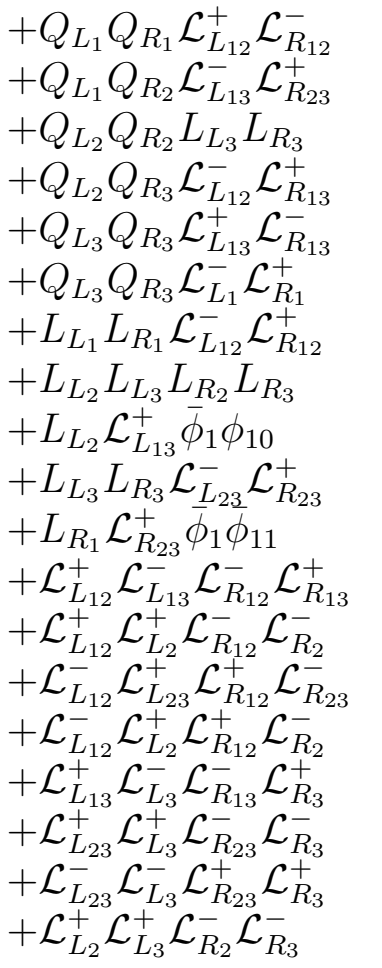

$+Q_{L_{1}} Q_{R_{1}} \mathcal{L}_{L_{12}}^{-} \mathcal{L}_{R_{12}}^{+}$ $+Q_{L_{1}} Q_{R_{3}} \mathcal{L}_{L_{12}}^{+} \mathcal{L}_{R_{23}}^{-}$ $+Q_{L_{2}} Q_{R_{2}} \mathcal{L}_{L_{12}}^{+} \mathcal{L}_{R_{12}}^{-}$ $+Q_{L_{3}} Q_{R_{1}} \mathcal{L}_{L_{23}}^{+} \mathcal{L}_{R_{12}}^{-}$ $+Q_{L_{3}} Q_{R_{3}} \mathcal{L}_{L_{13}}^{-} \mathcal{L}_{R_{13}}^{+}$ $+Q_{L_{3}} Q_{R_{3}} \mathcal{L}_{L_{2}}^{+} \mathcal{L}_{R_{2}}^{-}$ $+L_{L_{1}} L_{R_{1}} \mathcal{L}_{L_{3}}^{+} \mathcal{L}_{R_{3}}^{-}$ $+L_{L_{2}} L_{R_{2}} \mathcal{L}_{L_{12}}^{+} \mathcal{L}_{R_{12}}^{-}$ $+L_{L_{2}} \mathcal{L}_{L_{13}}^{+} \bar{\phi}_{2} \phi_{11}$ $+L_{L_{3}} L_{R_{3}} \mathcal{L}_{L_{1}}^{+} \mathcal{L}_{R_{1}}^{-}$ $+L_{R_{1}} \mathcal{L}_{R_{23}}^{+} \bar{\phi}_{2} \bar{\phi}_{10}$ $+\mathcal{L}_{L_{12}}^{+} \mathcal{L}_{L_{23}}^{+} \mathcal{L}_{R_{12}}^{-} \mathcal{L}_{R_{23}}^{-}$ $+\mathcal{L}_{L_{12}}^{+} \mathcal{L}_{L_{2}}^{+} \mathcal{L}_{R_{13}}^{-} \mathcal{L}_{R_{3}}^{-}$ $+\mathcal{L}_{L_{12}}^{-} \mathcal{L}_{L_{23}}^{-} \mathcal{L}_{R_{12}}^{+} \mathcal{L}_{R_{23}}^{+}$ $+\mathcal{L}_{L_{12}}^{-} \mathcal{L}_{L_{2}}^{-} \mathcal{L}_{R_{12}}^{+} \mathcal{L}_{R_{2}}^{+}$ $+\mathcal{L}_{L_{13}}^{-} \mathcal{L}_{L_{1}}^{+} \mathcal{L}_{R_{23}}^{+} \mathcal{L}_{R_{2}}^{-}$ $+\mathcal{L}_{L_{23}}^{+} \mathcal{L}_{L_{3}}^{-} \mathcal{L}_{R_{23}}^{-}{ }_{R_{R}}^{+} \mathcal{L}_{3}^{+}$ $+\mathcal{L}_{L_{1}}^{+} \mathcal{L}_{L_{3}}^{+} \mathcal{L}_{R_{1}}^{-} \mathcal{L}_{R_{3}}^{-}$ $+\mathcal{L}_{L_{2}}^{+} \mathcal{L}_{L_{3}}^{-} \mathcal{L}_{R_{2}}^{-} \mathcal{L}_{R_{3}}^{+}$

$$
\begin{aligned}
& +Q_{L_{1}} Q_{R_{1}} \mathcal{L}_{L_{3}}^{+} \mathcal{L}_{R_{3}}^{-} \\
& +Q_{L_{2}} Q_{L_{3}} Q_{R_{2}} Q_{R_{3}} \\
& +Q_{L_{2}} Q_{R_{2}} \mathcal{L}_{L_{12}}^{-} \mathcal{L}_{R_{12}}^{+} \\
& +Q_{L_{3}} Q_{R_{2}} \mathcal{L}_{L_{13}}^{-} \mathcal{L}_{R_{12}}^{+} \\
& +Q_{L_{3}} Q_{R_{3}} \mathcal{L}_{L_{23}}^{+} \mathcal{L}_{R_{23}}^{-} \\
& +Q_{L_{3}} Q_{R_{3}} \mathcal{L}_{L_{2}}^{-} \mathcal{L}_{R_{2}}^{+} \\
& +L_{L_{1}} L_{R_{1}} \mathcal{L}_{L_{3}}^{-} \mathcal{L}_{R_{3}}^{+} \\
& +L_{L_{2}} L_{R_{2}} \mathcal{L}_{L_{12}}^{-} \mathcal{L}_{R_{12}}^{+} \\
& +L_{L_{3}} L_{R_{3}} \mathcal{L}_{L_{13}}^{+} \mathcal{L}_{R_{13}}^{-} \\
& +L_{L_{3}} L_{R_{3}} \mathcal{L}_{L_{1}}^{-} \mathcal{L}_{R_{1}}^{+} \\
& +L_{R_{2}} \mathcal{L}_{R_{13}}^{-} \phi_{1} \bar{\phi}_{10} \\
& +\mathcal{L}_{L_{12}}^{+} \mathcal{L}_{L_{23}}^{-} \mathcal{L}_{R_{12}}^{-} \mathcal{L}_{R_{23}}^{+} \\
& +\mathcal{L}_{L_{12}}^{+} \mathcal{L}_{L_{2}}^{-} \mathcal{L}_{R_{12}}^{-} \mathcal{L}_{R_{2}}^{+} \\
& +\mathcal{L}_{L_{12}}^{-} \mathcal{L}_{L_{1}}^{+} \mathcal{L}_{R_{12}}^{+} \mathcal{L}_{R_{1}}^{-} \\
& +\mathcal{L}_{L_{13}}^{+} \mathcal{L}_{L_{1}}^{+} \mathcal{L}_{R_{23}}^{-} \mathcal{L}_{R_{2}}^{-} \\
& +\mathcal{L}_{L_{13}}^{-} \mathcal{L}_{L_{3}}^{+} \mathcal{L}_{R_{13}}^{+} \mathcal{L}_{R_{3}}^{-} \\
& +\mathcal{L}_{L_{23}}^{-} \mathcal{L}_{L_{2}}^{+} \mathcal{L}_{R_{13}}^{+} \mathcal{L}_{R_{1}}^{-} \\
& +\mathcal{L}_{L_{1}}^{+} \mathcal{L}_{L_{3}}^{-} \mathcal{L}_{R_{1}}^{-} \mathcal{L}_{R_{3}}^{+} \\
& +\mathcal{L}_{L_{2}}^{-} \mathcal{L}_{L_{3}}^{+} \mathcal{L}_{R_{2}}^{+} \mathcal{L}_{R_{3}}^{-} \\
& \quad
\end{aligned}
$$

$W_{4}($ singlets $), W_{4}($ mixed $), W_{4}($ hidden $)$ : none 


\section{Models with enhanced non-Abelian symmetries}

We next turn to our second example, Model 2. The boundary condition basis vectors and one-loop phases, which define the model, are given in Table (5.1) and matrix (5.2), respectively.

LRS Model 2 Boundary Conditions:

\begin{tabular}{|c|c|c|c|c|c|c|c|c|c|}
\hline & $\psi^{\mu}$ & $\chi^{12}$ & $\chi^{34}$ & $\chi^{56}$ & $\bar{\psi}^{1, \ldots, 5}$ & $\bar{\eta}^{1}$ & $\bar{\eta}^{2}$ & $\bar{\eta}^{3}$ & $\bar{\phi}^{1, \ldots, 8}$ \\
\hline$\alpha$ & 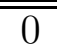 & $\overline{0}$ & $\overline{0}$ & $\overline{0}$ & 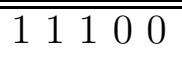 & 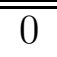 & 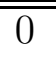 & 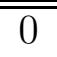 & $\begin{array}{lllllllll} & 1 & 1 & 1 & 1 & 0 & 0 & 0 & 0\end{array}$ \\
\hline$\beta$ & 0 & 0 & 0 & 0 & 11100 & 0 & 0 & 0 & 111100000 \\
\hline$\gamma$ & 0 & 0 & 0 & 0 & $\frac{1}{2} \quad \frac{1}{2} \quad \frac{1}{2} \quad 00$ & $\frac{1}{2}$ & $\frac{1}{2}$ & $\frac{1}{2}$ & \\
\hline
\end{tabular}

\begin{tabular}{c|cccc|cccc|cccc} 
& $y^{3} y^{6} y^{4} \bar{y}^{4} y^{5} \bar{y}^{5} \bar{y}^{3} \bar{y}^{6}$ & \multicolumn{2}{|c|}{$y^{1} \omega^{5} y^{2} \bar{y}^{2} \omega^{6} \bar{\omega}^{6} \bar{y}^{1} \bar{\omega}^{5}$} & $\omega^{2} \omega^{4} \omega^{1} \bar{\omega}^{1} \omega^{3} \bar{\omega}^{3} \bar{\omega}^{2} \bar{\omega}^{4}$ \\
\hline \hline$\alpha$ & 1 & 0 & 0 & 0 & 0 & 0 & 1 & 1 & 0 & 0 & 1 & 1 \\
$\beta$ & 0 & 0 & 1 & 1 & 1 & 0 & 0 & 0 & 0 & 1 & 0 & 1 \\
$\gamma$ & 0 & 0 & 1 & 0 & 1 & 0 & 0 & 1 & 0 & 1 & 0 & 0
\end{tabular}

LRS Model 2 Generalized GSO Coefficients:

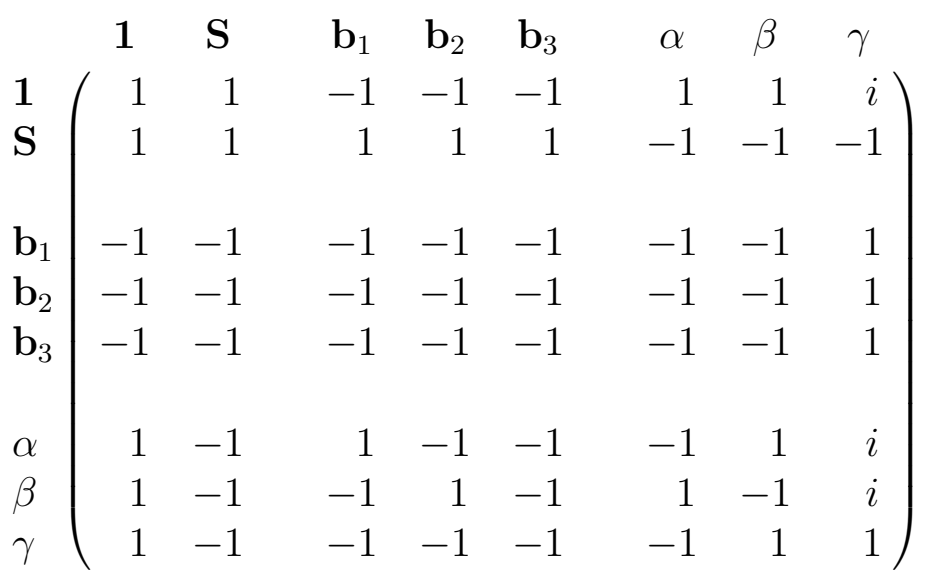

Model 2, defined by Table (5.1) and matrix (5.2), differs from Model 1 only in the boundary conditions of the hidden sector world-sheet fermions $\left\{\bar{\phi}^{1, \cdots, 8}\right\}$ in the basis vectors $\beta$ and $\gamma$, and in the GSO phases beyond the NAHE ones. In the basis vector $\beta$ the alterations are $\beta_{\bar{\phi}^{3,4}}=0 \rightarrow 1 ; \beta_{\bar{\phi}^{5,6}}=1 \rightarrow 0$ and in $\gamma$ they are $\gamma_{\bar{\phi}^{1}}=1 \rightarrow \frac{1}{2} ; \gamma_{\bar{\phi}^{4}}=\frac{1}{2} \rightarrow 1$. While the spectrum arising from the NAHE set remains essentially unaltered, the spectrum arising from the basis vectors beyond the NAHE set is substantially modified. Hence some phenomenological features of the two models are significantly modified.

The total gauge group of Model 2 arises as follows. In the observable sector the NS boundary conditions produce the generators of $\left(S U(3)_{C} \times U(1)_{C} \times S U(2)_{L} \times S U(2)_{R} \in\right.$ 
$S O(10)) \times U(1)_{1,2,3} \times U(1)_{4,5,6}$, while in the hidden sector the NS boundary conditions produce the generators of

$$
S U(3)_{H_{1}} \times U(1)_{H_{1}} \times U(1)_{7} \times S U(3)_{H_{2}} \times U(1)_{H_{2}} \times U(1)_{8} .
$$

$U(1)_{H_{1}}$ and $U(1)_{H_{2}}$ correspond to the combinations of the world-sheet charges

$$
\begin{aligned}
Q_{H_{1}} & =Q\left(\bar{\phi}^{1}\right)-Q\left(\bar{\phi}^{2}\right)-Q\left(\bar{\phi}^{3}\right)+\sum_{i=4}^{7} Q(\bar{\phi})^{i}-Q(\bar{\phi})^{8}, \\
Q_{H_{2}} & =\sum_{i=1}^{4} Q(\bar{\phi})^{i}-Q\left(\bar{\phi}^{7}\right)-Q(\bar{\phi})^{8} .
\end{aligned}
$$

and $U(1)_{7,8}$ arise from the world-sheet currents $\bar{\phi}^{4} \bar{\phi}^{4^{*}}$ and $\bar{\phi}^{8} \bar{\phi}^{8^{*}}$, respectively. The sector $\zeta \equiv 1+\mathbf{b}_{1}+\mathbf{b}_{2}+\mathbf{b}_{3}$ produces the representations $(3,1)_{-5,0} \oplus(\overline{3}, 1)_{5,0}$ and $(1,3)_{0,-5} \oplus(1, \overline{3})_{0,5}$ of $S U(3)_{H_{1}} \times U(1)_{H_{1}}$ and $S U(3)_{H_{2}} \times U(1)_{H_{2}}$. Thus, the $E_{8}$ symmetry reduces to $S U(4)_{H_{1}} \times S U(4)_{H_{2}} \times U(1)^{2}$. The additional $U(1)^{\prime}$ 's in $S U(4)_{H_{1,2}}$ are given by the combinations in eqs. (5.4) and (5.5), respectively. The remaining $U(1)$ symmetries in the hidden sector, $U(1)_{7^{\prime}}$ and $U(1)_{8^{\prime}}$, correspond to the combination of world-sheet charges

$$
\begin{aligned}
& Q_{7^{\prime}}=Q\left(\bar{\phi}^{4}\right)+Q\left(\bar{\phi}^{8}\right), \\
& Q_{8^{\prime}}=-\sum_{i=1}^{3} Q(\bar{\phi})^{i}+Q\left(\bar{\phi}^{4}\right)-\sum_{i=5}^{7} Q(\bar{\phi})^{i}-Q(\bar{\phi})^{8} .
\end{aligned}
$$

Model 2 contains two combinations of non-NAHE basis vectors with $\mathbf{X}_{L} \cdot \mathbf{X}_{L}=0$, which therefore may give rise to additional space-time vector bosons. The first is the vector combination $\beta \pm \gamma$. The second combination is given by $\zeta+2 \gamma$, where $\zeta \equiv 1+\mathbf{b}_{1}+\mathbf{b}_{2}+\mathbf{b}_{3}$. The presence of the first combination depends on the assignment of periodic boundary conditions in the basis vectors $\alpha, \beta$ and $\gamma$, which extend the NAHE set and is therefore model dependent. The second combination, however, arises only from the NAHE set basis vectors plus $2 \gamma$ and is therefore independent of the assignment of periodic boundary conditions in the basis vectors $\alpha, \beta$ and $\gamma$. This vector combination is therefore generic for the pattern of symmetry breaking $S O(10) \rightarrow S U(3)_{C} \times U(1)_{C} \times S U(2)_{L} \times S U(2)_{R}$, in NAHE based models.

In Model 2 all the space-time vector bosons from the sector $\beta \pm \gamma$ are projected out by the GSO projections and therefore give no gauge enhancement. The sector $\zeta+2 \gamma$, however, gives rise to two additional space-time vector bosons which are charged with respect to the world-sheet $U(1)$ currents. This enhances one of the world-sheet $U(1)$ combinations to $S U(2)_{\text {cust }}$. The relevant combination of world-sheet charges is given by

$$
Q_{S U(2)_{C}}=Q_{C}+\left(Q_{\eta_{1}}+Q_{\eta_{2}}+Q_{\eta_{3}}\right)-Q_{7^{\prime}}
$$


The remaining orthogonal $U(1)$ combinations are

$$
\begin{aligned}
Q_{1^{\prime}} & =Q_{1}-Q_{2}, \\
Q_{2^{\prime}} & =Q_{1}+Q_{2}-2 Q_{3}, \\
Q_{3^{\prime}} & =-Q_{C}+Q_{1}+Q_{2}+Q_{3}+Q_{8^{\prime}} \\
Q_{7^{\prime \prime}} & =Q_{C}+Q_{1}+Q_{2}+Q_{3}+3 Q_{7^{\prime}} \\
Q_{8^{\prime \prime}} & =-4 Q_{C}+4\left(Q_{1}+Q_{2}+Q_{3}\right)-3 Q_{8^{\prime}} .
\end{aligned}
$$

and $Q_{4,5,6}$ are unchanged. Thus, the full massless spectrum transforms under the final gauge group, $S U(3)_{C} \times S U(2)_{L} \times S U(2)_{R} \times S U(2)_{\text {cust }} \times U(1)_{1^{\prime}, 2^{\prime}, 3^{\prime}} \times U(1)_{4,5,6} \times$ $S U(4)_{H_{1}} \times S U(4)_{H_{2}} \times U(1)_{7^{\prime \prime}, 8^{\prime \prime}}$.

In addition to the graviton, dilaton, antisymmetric sector and spin-1 gauge bosons, the NS sector gives two pairs of electroweak doublets, transforming as $(1,2,2,1)$ under $S U(3)_{C} \times S U(2)_{L} \times S U(2)_{R} \times S U(2)_{\text {cust }}$; three pairs of $S O(10)$ singlets with $U(1)_{1,2,3}$ charges; and three singlets of the entire four dimensional gauge group. The sector $\mathbf{S}+\mathbf{b}_{1}+\mathbf{b}_{2}+\alpha+\beta$ produces one pair of $S U(2)_{\text {cust }}$ doublets that can be used to break the $S U(2)_{\text {cust }}$ symmetry, and three pairs of non-Abelian singlets with $U(1)_{1,2,3}$ charges.

The states from the sectors $\mathbf{b}_{j} \oplus \zeta+2 \gamma(j=1,2,3)$ produce the three light generations. The states from these sectors and their decomposition under the entire gauge group are shown in Table 2. The leptons (and quarks) are singlets of the color $S U(4)_{H_{1}, H_{2}}$ gauge groups and the $U(1)_{7^{\prime \prime}}$ symmetry of eq. (5.9) becomes a gauged leptophobic symmetry. The remaining massless states and their quantum numbers are also given in Table 2 in Appendix C. We also provide, in Appendix D, the Model 2 cubic through quintic order superpotential terms.

\subsection{Definition of the weak-hypercharge}

We now turn to the definition of the weak-hypercharge in this LRS model. Due to the enhanced symmetry there are several possibilities to define a weak-hypercharge combination which is still family universal and reproduces the correct charge assignment for the Standard Model fermions. As we discuss below, this feature of the free fermionic models with enhanced symmetry presents an interesting way to understand how the Standard Model spectrum may still arise from $S O(10)$ representation, i.e. from the three 16's of $S O(10)$ which arise from the NAHE set, while the weakhypercharge does not possess the canonical $S O(10)$ embedding. We remark that this type of enhanced symmetry also plays a roll in forbidding operators which mediate proton decay. We also note that LRS models without the canonical $S O(10)$ embedding of the weak-hypercharge have also been recently discussed in the framework of Type I string constructions [19]. There it was argued that the non-canonical embedding of the weak-hypercharge is advantageous for obtaining coupling unification in that framework. In the heterotic string the natural unification scale is of course the 
GUT or the heterotic string scale, and therefore the natural embedding of the weakhypercharge is the canonical one. Nevertheless, as we stated above, the main aim of our exercise here is to demonstrate how the Standard Model spectrum may still arise from $S O(10)$ representations while the normalization of the weak-hypercharge (and consequently the Weinberg angle at the unification scale) do not have the canonical $S O(10)$ value. The usefulness of this result to string models with a lower unification scale will then depend on improved understanding of the duality relation between the various models and the properties which are maintained in an extrapolation from weak to strong coupling. That is, one can imagine that a property of the type we describe here will have its correspondence also in the dual Type I models.

One option is to define the weak-hypercharge with the standard $S O(10)$ embedding, as in eq. (5.10),

$$
U(1)_{Y}=\frac{1}{3} U(1)_{C}+\frac{1}{2} U(1)_{L}
$$

This is identical to the weak-hypercharge definition in $S U(3) \times S U(2) \times U(1)_{Y}$ free fermionic models, which do not have enhanced symmetries. However, in the present model, the $U(1)_{C}$ symmetry is now part of the extended custodial symmetry $S U(2)_{\text {cust }}$. Expressing $U(1)_{C}$ in terms of the new linear combinations defined above, we have

$$
\frac{1}{3} U(1)_{C}=\frac{1}{6}\left\{\frac{1}{4}\left(3 T_{\text {cust }}^{3}+U_{7^{\prime \prime}}\right)-\frac{1}{7}\left(3 U_{3^{\prime}}+U_{8^{\prime \prime}}\right)\right\} .
$$

Thus $U(1)_{Y}$, by depending on $T_{\text {cust }}^{3}$, is no longer orthogonal to $S U(2)_{\text {cust }}$. We must therefore instead define the new linear combination with this term removed,

$$
\begin{aligned}
U(1)_{Y^{\prime}} & \equiv U(1)_{Y}-\frac{1}{2} T^{3} \\
= & \frac{1}{2} U(1)_{L}+\frac{5}{24} U(1)_{C} \\
& \quad-\frac{1}{8}\left[U(1)_{1}+U(1)_{2}+U(1)_{3}-U(1)_{7}-U(1)_{9}\right]
\end{aligned}
$$

so that the weak-hypercharge is expressed in terms of $U(1)_{Y^{\prime}}$ as

$$
U(1)_{Y}=U(1)_{Y^{\prime}}+\frac{1}{2} T_{\text {cust }}^{3} \quad \Longrightarrow \quad Q_{\text {e.m. }}=T_{L}^{3}+Y=T_{L}^{3}+Y^{\prime}+\frac{1}{2} T_{\text {cust }}^{3} .
$$

The final observable gauge group then takes the form

$S U(3)_{C} \times S U(2)_{L} \times S U(2)_{R} \times S U(2)_{\text {cust }} \times U(1)_{Y^{\prime}} \times\{$ seven other $U(1)$ factors $\}$.

The remaining seven $U(1)$ factors must be chosen as linear combinations of the previous $U(1)$ factors so as to be orthogonal to the each of the other factors in (5.14). 
Next we discuss the Kač-Moody factors associated with the $U(1)$ factors in this model. In this class of string models, the Kač-Moody level of the non-Abelian group factor is always one. The situation is somewhat more complicated for the $U(1)$ factors, however. In general, a given $U(1)$ current $U$ will be a combination of the simple worldsheet $U(1)$ currents $U_{f} \equiv f^{*} f$ corresponding to individual worldsheet fermions $f$, and will take the form $U=\sum_{f} a_{f} U_{f}$ where the $a_{f}$ are certain model-specific coefficients. The $U_{f}$ are each individually normalized to one, so that $\left\langle U_{f}, U_{f}\right\rangle=1$. To produce the correct conformal dimension for the massless states, each of the $U(1)$ linear combinations $U$ must also be normalized to one. The proper normalization coefficient for the linear combination $U$ is thus given by $N=\left(\sum_{f} a_{f}^{2}\right)^{-1 / 2}$, so that the properly normalized $U(1)$ current $\hat{U}$ is given by $\hat{U}=N \cdot U$.

Now in general, the Kač-Moody level of the $U(1)_{Y}$ generator can be deduced from the OPE's between two of the $U(1)$ currents, and will be

$$
k_{1}=2 N^{-2}=2 \sum_{f} a_{f}^{2} .
$$

For a weak-hypercharge that is a combination of several $U(1)$ 's with different normalizations, the result (5.15) generalizes to

$$
k_{1}=\sum_{i} a_{i}^{2} k_{i}
$$

where the $k_{i}$ are the individual normalizations for each of the $U(1)$ 's.

In Model 1, the $U(1)_{Y}$ generator is given as a combination of simple worldsheet currents that produces the correct weak-hypercharges for the Standard Model particles. Thus, in that case, $k_{1}$ is simply given by (5.15). However, for the weakhypercharges (5.12) and (5.13) that appear in Model 2 we instead use (5.16). Hence, for this weak-hypercharge, we see from (5.13) and (5.16) that $k_{1}=(1 / 4) k_{2_{C}}+k_{Y^{\prime}}=$ $1 / 4+17 / 12=5 / 3$, which is the same as the standard $S O(10)$ normalization.

Alternatively, we can define the weak-hypercharge to be the combination

$$
U(1)_{Y}=\frac{1}{2} U(1)_{L}-\frac{1}{6} U(1)_{3^{\prime}}+\frac{1}{6} U_{7^{\prime \prime}}
$$

where $U(1)_{3^{\prime}}$ and $U(1)_{7^{\prime \prime}}$ are given in (5.9). This combination still reproduces the correct charge assignment for the Standard Model states. In this case the Kač-Moody levels of $U(1)_{L}, U(1)_{3^{\prime}}$ and $U(1)_{7^{\prime \prime}}$ are 4,28 and 48 respectively, so that $k_{Y}=28 / 9$. Therefore, the Weinberg angle at the unification scale is $\sin ^{2} \theta_{W}=0.243$. Naturally, the point that we want to raise is not that the present model with this value of $\sin ^{2} \theta_{W}$ provides a realistic unified model. Rather, we make the following interesting observation: The three Standard Model generations still arise from $S O(10)$ representations. Specifically, the Standard Model three generations all arise from the three $\mathbf{1 6}$ representations of $S O(10)$ of the NAHE set basis vectors. However, the weak-hypercharge does not possess the standard $S O(10)$ embedding and consequently, $\sin ^{2} \theta_{W} \neq 3 / 8$ at 
the unification scale. Of course, it will be of further interest to see if such a structure can also emerge from Type I string constructions which actually allow for a lower unification scale. The results of ref. [19], which show that some of the structure of compactifying the heterotic string on a particular orbifold, is actually preserved also in the Type I models, give rise to the suspicion that this may indeed be the case.

\section{Anomalous $U(1)$}

A general property of the realistic free fermionic heterotic string models, which is also shared by many other superstring vacua, is the existence of an "anomalous" $U(1)$. The presence of an Abelian anomalous symmetry in superstring derived models yields many desirable phenomenological consequences from the point of view of the effective low energy field theory. Indeed, the existence of such an anomalous $U(1)$ symmetry in string derived models has inspired vigorous attempts to understand numerous issues, relevant for the observable phenomenology, including: the fermion mass spectrum, supersymmetry breaking cosmological implications, and more. From the perspective of string phenomenology an important function of the anomalous $U(1)$ is to induce breaking and rank reduction of the four dimensional gauge group. In general, the existence of an anomalous $U(1)$ in a string model implies that the string vacuum is unstable and must be shifted to a stable point in the moduli space. This arises because, by the Green-Schwarz anomaly cancellation mechanism, the anomalous $U(1)$ gives rise to a Fayet-Iliopoulos term which breaks supersymmetry. Supersymmetry is restored and the vacuum is stabilized by sliding the vacuum along flat $F$ and $D$ directions. This is achieved by assigning non-vanishing VEVs to some scalar fields in the massless string spectrum.

An important issue in string phenomenology is therefore to understand what are the general conditions for the appearance of an anomalous $U(1)$ and under what conditions an anomalous $U(1)$ is absent. The previously studied realistic free fermionic string models, which include the FSU5, PS, and SLM types, have always contained an anomalous $U(1)$ symmetry. In contrast, in the two LRS models defined respectively by (4.1,4.2) and (5.1,5.2) all the $U(1)$ symmetries in the four dimensional gauge group are anomaly free. This is, in fact, the first instance that realistic three generation $(2,0)$ heterotic string models have produced models which do not contain an anomalous $U(1)$ symmetry. Irrespective of the potential phenomenological merit of an anomalous $U(1)$ symmetry, it is important to extract the properties of the models that result in the presence, or the absence, of an anomalous $U(1)$ symmetry.

For completeness we first discuss the case of the free fermionic models which contain an anomalous $U(1)$, i.e., the FSU5, the PS, and the SLM string models. The question of the anomalous $U(1)$ symmetry in string models, in general, and in the free fermionic models, in particular, was studied in some detail in ref. [12]. The anomalous $U(1)$ in the free fermionic models is in general a combination of two distinct kinds of world-sheet $U(1)$ currents, those generated by $\bar{\eta}^{j}$ and those 
generated by the additional complexified fermions from the set $\{\bar{y}, \bar{\omega}\}^{1, \cdots, 6}$. The trace of the $U(1)$ charges of the entire massless string spectrum can then be non-vanishing under some of these world-sheet $U(1)$ currents. One combination of these $U(1)$ currents then becomes the anomalous $U(1)$, whereas all the orthogonal combinations are anomaly free. To understand the origin of the anomalous $U(1)$ in the realistic free fermionic models, it is instructive to consider the contributions from the two types of world-sheet $U(1)$ currents separately.

In ref. [12] it was shown that the anomalous $U(1)$ in the realistic free fermionic models can be seen to arise due to the breaking of the world-sheet supersymmetry from $(2,2)$ to $(2,0)$. Consider the set of boundary condition basis vectors $\left\{\mathbf{1}, \mathbf{S}, \zeta, \mathbf{X}, \mathbf{b}_{1}, \mathbf{b}_{2}\right\}$ [12], which produces (for an appropriate choice of the GSO phases) the model with $S O(12) \times E_{6} \times U(1)^{2} \times E_{8}$ gauge group. It was shown that if we choose the GSO phases such that $E_{6} \rightarrow S O(10) \times U(1)$, the $U(1)$ in the decomposition of $E_{6}$ under $S O(10) \times U(1)$ becomes the anomalous $U(1)$. This $U(1)$ is produced by the combination of world-sheet currents $\bar{\eta}^{1} \bar{\eta}^{1^{*}}+\bar{\eta}^{2} \bar{\eta}^{2^{*}}+\bar{\eta}^{3} \bar{\eta}^{3^{*}}$. We can view all of the realistic FSU5, PS, and SLM free fermionic string models as being related to this $S O(12) \times E_{6} \times U(1)^{2} \times E_{8}$ string vacuum. This combination of $U(1)$ currents therefore contributes to the anomalous $U(1)$ in all the realistic free fermionic models with FSU5, PS, or SLM gauge groups.

The existence of the anomalous $U(1)$ in the FSU5, PS, or SLM, and its absence in the LRS string models can be traced to different $N=4$ string vacua in four dimensions. While in the $E_{6}$ model one starts with an $N=4 S O(12) \times E_{8} \times E_{8}$ string vacua, produced by the set $\{\mathbf{1}, \mathbf{S}, \mathbf{X}, \zeta\}[12]$, we can view the FSU5, PS, and SLM string models as starting from an $N=4 S O(12) \times S O(16) \times S O(16)$ string vacua. In this case the two spinorial representations from the sectors $\mathbf{X}$ and $\zeta$, that complete the adjoint of $S O(16) \times S O(16)$ to $E_{8} \times E_{8}$, are projected out by the choice of the GSO projection phases. The subsequent projections, induced by the basis vectors $\mathbf{b}_{1}$ and $\mathbf{b}_{2}$, which correspond to the $\mathbb{Z}_{2} \times \mathbb{Z}_{2}$ orbifold twistings, then operate identically in the two models, producing in one case the $E_{6}$, and in the second case the $S O(10) \times U(1)$, gauge groups, respectively. The important point, however, is that both cases preserve the "standard embedding" structure which splits the observable and hidden sectors. The important set in this respect is the set $\{\mathbf{1}, \mathbf{S}, \mathbf{X}, \zeta\}$, where $\mathbf{X}$ has periodic boundary conditions for $\left\{\bar{\psi}^{1, \cdots, 5}, \bar{\eta}^{1}, \bar{\eta}^{2}, \bar{\eta}^{3}\right\}$. The choice of the phase $c\left(\begin{array}{c}\mathbf{x} \\ \zeta\end{array}\right)= \pm 1$ fixes the vacuum to $E_{8} \times E_{8}$ or $S O(16) \times S O(16)$.

In contrast, the LRS free fermionic string models do not start with the $N=4$ $E_{8} \times E_{8}$ or $S O(16) \times S O(16)$ vacua. Rather, in this case the starting $N=4$ vacua can be seen to arise from the set of boundary condition basis vectors $\{\mathbf{1}, \mathbf{S}, 2 \gamma, \mathbf{X}\}$. 
Starting with this set and with the choice of GSO projection phases

$$
\begin{aligned}
& \mathbf{1} \\
& \mathbf{S} \\
& \mathbf{X} \\
& 2 \gamma
\end{aligned}\left(\begin{array}{rrrr}
1 & \mathbf{S} & \mathbf{X} & 2 \gamma \\
1 & 1 & -1 & -1 \\
-1 & -1 & -1 & -1 \\
-1 & -1 & -1 & 1
\end{array}\right),
$$

the resulting string vacua has $N=4$ space-time supersymmetry with $S O(16) \times E_{7} \times$ $E_{7}$ gauge group. The sectors $\mathbf{b}_{1}$ and $\mathbf{b}_{2}$ are then added as in the previous models. The LRS string models therefore do not preserve the "standard embedding" splitting between the observable and hidden sectors. This is the first basic difference between the FSU5, PS, or SLM, and the LRS free fermionic models.

Now turn to the case of the three generation models. The chirality of the generations from the sectors $\mathbf{b}_{j}(j=1,2,3)$ is induced by the projection which breaks $N=2 \rightarrow N=1$ space-time supersymmetry. Chirality for the generations is therefore fixed by the GSO projection phase $c\left(\begin{array}{l}\mathbf{b}_{i} \\ \mathbf{b}_{j}\end{array}\right)$ with $i \neq j$. On the other hand, generation charges under $U(1)_{j}$ are fixed by the $\mathbf{X}$ projection in the $E_{6}$ model, by the projection induced by the vector $2 \gamma$ of the FSU5, PS, and SLM string models, or by the vector $2 \gamma$ of the LRS string models. The difference is that in the case of the FSU5, PS, and SLM string models the $2 \gamma$ projection fixes the same sign for the $U(1)_{j}$ charges of the states from the sectors $\mathbf{b}_{j}$. In contrast, in the LRS free fermionic models the corresponding $2 \gamma$ projection fixes one sign for the $\left(Q_{R}+L_{R}\right)_{j}$ states and the opposite sign for the $\left(Q_{L}+L_{L}\right)_{j}$ states. The consequence is that the total trace vanishes and the sectors $\mathbf{b}_{j}$ do not contribute to the trace of the $U(1)_{j}$ charges. This is in fact the reason that LRS free fermionic models can appear without an anomalous $U(1)$.

We stress that the existence of LRS free fermionic string models without an anomalous $U(1)$ does not preclude the possibility of other LRS models with an anomalous $U(1)$. Our Model 3, specified by Table (6.2) and matrix (6.3) provides a counter-example.

\begin{tabular}{|c|c|c|c|c|c|c|c|c|c|}
\hline & $\psi^{\mu}$ & $\chi^{12}$ & $\chi^{34}$ & $\chi^{56}$ & $\bar{\psi}^{1, \ldots, 5}$ & $\bar{\eta}^{1}$ & $\bar{\eta}^{2}$ & $\bar{\eta}^{3}$ & $\bar{\phi}^{1, \ldots, 8}$ \\
\hline $\bar{\alpha}$ & 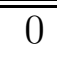 & $\overline{0}$ & $\overline{0}$ & $\overline{0}$ & 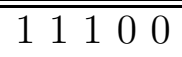 & 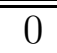 & 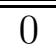 & 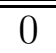 & $\begin{array}{lllllllll}1 & 1 & 1 & 0 & 0 & 0 & 0\end{array}$ \\
\hline$\beta$ & 0 & 0 & 0 & 0 & 11100 & 0 & 0 & 0 & 11110000 \\
\hline$\gamma$ & 0 & 0 & 0 & 0 & $\begin{array}{lllll}\frac{1}{2} & \frac{1}{2} & \frac{1}{2} & 0 & 0\end{array}$ & $\frac{1}{2}$ & $\frac{1}{2}$ & $\frac{1}{2}$ & \\
\hline
\end{tabular}

LRS Model 3 Boundary Conditions:

\begin{tabular}{c|cccc|rrrr|rrrr} 
& \multicolumn{3}{|c|}{$y^{3} y^{6}$} & $y^{4} \bar{y}^{4}$ & $y^{5} \bar{y}^{5}$ & $\bar{y}^{3} \bar{y}^{6}$ & \multicolumn{1}{|c|}{$y^{1} \omega^{5} y^{2} \bar{y}^{2} \omega^{6} \bar{\omega}^{6} \bar{y}^{1} \bar{\omega}^{5}$} & $\omega^{2} \omega^{4} \omega^{1} \bar{\omega}^{1} \omega^{3} \bar{\omega}^{3} \bar{\omega}^{2} \bar{\omega}^{4}$ \\
\hline \hline$\alpha$ & 1 & 1 & 1 & 0 & 1 & 1 & 1 & 0 & 1 & 1 & 1 & 0 \\
$\beta$ & 0 & 1 & 0 & 1 & 0 & 1 & 0 & 1 & 1 & 0 & 0 & 0 \\
$\gamma$ & 0 & 0 & 1 & 1 & 1 & 0 & 0 & 0 & 0 & 1 & 0 & 1
\end{tabular}


LRS Model 3 Generalized GSO Coefficients:

\begin{tabular}{|c|c|c|c|c|c|c|c|c|}
\hline & 1 & $\mathbf{S}$ & $\mathbf{b}_{1}$ & $\mathbf{b}_{2}$ & $\mathbf{b}_{3}$ & $\alpha$ & $\beta$ & $\gamma$ \\
\hline 1 & 1 & 1 & -1 & -1 & -1 & 1 & 1 & $i$ \\
\hline$S$ & 1 & 1 & 1 & 1 & 1 & -1 & -1 & -1 \\
\hline $\mathbf{b}_{1}$ & -1 & -1 & -1 & -1 & -1 & -1 & -1 & $i$ \\
\hline $\mathbf{b}_{2}$ & -1 & -1 & -1 & -1 & -1 & -1 & -1 & $i$ \\
\hline $\mathbf{b}_{3}$ & -1 & -1 & -1 & -1 & -1 & -1 & 1 & $i$ \\
\hline$\alpha$ & 1 & -1 & 1 & 1 & 1 & 1 & 1 & 1 \\
\hline$\beta$ & 1 & -1 & -1 & -1 & 1 & -1 & -1 & -1 \\
\hline$\gamma$ & 1 & -1 & 1 & -1 & 1 & -1 & -1 & 1 \\
\hline
\end{tabular}

Similar to our Models 1 and 2, Model 3 uses the LRS breaking pattern. It also contains three generations from the sectors $\mathbf{b}_{1}, \mathbf{b}_{2}$ and $\mathbf{b}_{3}$, and the untwisted spectrum is similar to that of the previous two models. However, Model 3 actually contains three anomalous $U(1)$ symmetries: $\operatorname{Tr} U_{4}=-24, \operatorname{Tr} U_{5}=-24, \operatorname{Tr} U_{6}=-24$, one combination of which,

$$
U(1)_{A}=U_{4}+U_{5}+U_{6},
$$

is anomalous, while two orthogonal combinations are anomaly free. In this model we see that the anomalous $U(1)$ 's correspond to $U(1)$ symmetries which arise from the additional complexified world-sheet fermions in the set $\{\bar{y}, \bar{\omega}\}^{1, \cdots, 6}$. This is in agreement with the above argument that the $U(1)_{j=1,2,3}$, which are generated by the $\bar{\eta}^{j}$ world-sheet fermions, are anomaly free in the LRS free fermionic string models. The potential implications for the flavor mass spectrum are of particular interest in this regard.

To close the discussion on the anomalous $U(1)$ in the the LRS string models we remark that in ref. [12] the general conditions that forbid the appearance of anomalous $U(1)$ in string models were discussed. Theorem 3, part (b), of [12] allows us to prove, without computing the trace of the charge $\operatorname{Tr} Q^{i}$, for each $U(1)_{i}$, that all of the Abelian gauge groups in a given model are anomaly free. Theorem 3 states that:

A model is completely free of anomalous $U(1)$ if, for each $U(1)_{i}$, there is at least one simple gauge group $\mathcal{G}$ for which (a) all non-trivial massless reps of $\mathcal{G}$ do not carry $U(1)_{i}$ charge, or (b) the trace of $Q_{i}$ over all massless non-trivial reps of $\mathcal{G}$ is zero.

In Model 1 the only two non-trivial representations of the hidden sector $S U(3)_{H_{1}}$ gauge group is the vector-like pair of fields, $H_{1}$ and $\bar{H}_{1}$. Thus, the trace of each $U(1)_{i}$ charge over $S U(3)_{H_{1}}$ reps is clearly zero. This implies, by Theorem 3(b), that all of the $U(1)_{i}$ in this model are anomaly free. (Note that the vector-like fields $H_{2}$ and $\bar{H}_{2}$ of $S U(3)_{H_{2}}$ imply this also. Furthermore, the same method of proof also applies to Model 2 when we replace $S U(3)_{H_{1}, H_{2}}$ with $S U(4)_{H_{1}, H_{2}}$.) 


\section{Phenomenological discussion and conclusions}

Clearly the most striking new feature of the LRS string models that we presented is the absence of an anomalous $U(1)$ symmetry in two of them. In the past much of the analysis of the three generation free fermionic models involved the analysis of flat directions that are induced by the cancelation of the anomalous $U(1)$ D-term [2, 20]. The Standard Model singlet VEVs that are used to cancel this D-term spontaneously break some of the additional $U(1)$ symmetries in the string models. Such singlet VEVs are necessitated by the requirement that the superstring vacuum preserves supersymmetry at the string scale. An important issue in this regard is therefore whether for such flat directions there exist one, or perhaps several, $U(1)$ combinations which remain unbroken. $U(1)$ combinations that remain unbroken down to sufficiently low energies may give rise to interesting observable effects. In contrast, the absence of an anomalous $U(1)$ in the LRS string models that we discussed here allows, in principle, all the extra $U(1)$ 's to remain unbroken at the string scale. Of course imposing some phenomenological constraints, like the decoupling of fractionally charged states, may force some Standard Model singlet fields to acquire a non-vanishing VEV. In which case the analysis of the flat directions is reintroduced. However, this is not necessitated by the requirement that the anomalous $U(1) \mathrm{D}$-term vanishes, and hence not by the requirement that the vacuum preserves supersymmetry. Similarly, the Fayet-Iliopoulos term which is induced by the anomalous $U(1)$ [21] gives rise to an order parameter which, together with the flat direction singlet VEVs, is used to produce the hierarchical fermion mass pattern. This order parameter is therefore no longer present if there is no anomalous $U(1)$, as is the case in some of the LRS string models that we presented.

Examining the field content of Model 1, we note that the model contains the neutral Standard Model singlet component of the $\mathbf{1 6}$ of $S O(10)$. This field can therefore be used to break the $S U(2)_{R}$ gauge symmetry. However, it is noted that the corresponding component of the $\overline{\mathbf{1 6}}$ is absent from the model and all the remaining $S U(2)_{R}$ doublets have fractional electric charge with respect to the most natural definition of the weak-hypercharge give in eq. (5.10). The absence of the corresponding neutral component from the $\overline{\mathbf{1 6}}$ is a common feature in the three generation free fermionic models in which the $S O(1 O)$ is broken by at least two different basis vectors. Therefore, assuming that supersymmetry is not broken at a high scale, the $S U(2)_{R}$ symmetry cannot be broken along a supersymmetric flat directions, and can only be broken by a VEV that does not preserve supersymmetry at a lower scale. From Table 2 we note that Model 2 contains two $S U(2)_{C}$ doublets, $N_{\alpha \beta}$ and $\bar{N}_{\alpha \beta}$, from the sector $\mathbf{S}+\mathbf{b}_{1}+\mathbf{b}_{2}+\alpha+\beta$, that can be used to break the custodial $S U(2)$ symmetry along a flat direction.

As with Model 1, both Models 2 and 3 contain the neutral component of the $\mathbf{1 6}$ of $S O(10)$ that can be employed to break the $S U(2)_{R}$ symmetry. We remark that this conclusion holds for the definition of the weak-hypercharge as given in eq. (5.10). 
Other viable definitions of the weak-hypercharge may result in more electrically neutral fields that may be used to break $S U(2)_{R}$. As we discussed above, with such possible alternative definitions the Standard Model spectrum still arises from the 16 representation of $S O(1 O)$, but the weak-hypercharge normalization differs from the canonical $S O(10)$ normalization.

From Tables $1-3$ we note that all three models contain the required Higgs bidoublet representations, $h_{1}$ and $h_{2}$, that are needed in order to generate the Standard Model gauge boson and fermion masses. Examining the superpotential terms, eqs. (4.9) and (D.1), of Models 1 and 2 respectively, we note that the couplings $Q_{L_{i}} Q_{R_{i}} h_{i}$ and $L_{L_{i}} L_{R_{i}} h_{i}$ exist to provide potential mass terms for the states from the sectors $\mathbf{b}_{1}$ and $\mathbf{b}_{2}$. The structure of the basis vectors beyond the NAHE set, $\alpha, \beta$ and $\gamma$, which break the cyclic permutation symmetry between the three twisted sectors $\mathbf{b}_{1}, \mathbf{b}_{2}$ and $\mathbf{b}_{3}$, results in the states from the sector $\mathbf{b}_{3}$ being identified with the lightest generation. This outcome is similar to the result that was found in the case of the free fermionic SLM string models [22], and again is a reflection of the breaking of the cyclic permutation symmetry by the basis vectors $\alpha, \beta$ and $\gamma$.

¿From eq. (4.11) we note that in Model 1, provided that $\Phi_{3}$ gets a non-vanishing VEV of the order of the string scale, then the entire hidden matter spectrum of Model 1 becomes superheavy. In this case the content of the hidden sector spectrum consists of the gauge bosons of the unbroken hidden $E_{8}$ subgroup, which in model 1 is $S U(3) \times S U(3) \times U(1)^{4}$. These hidden states can interact with the observable sector only via the heavy hidden matter states, which are charged with respect to the horizontal $U(1)$ symmetries, $U(1)_{1,2}$. The observable sector states are also charged under the horizontal $U(1)_{1,2}$ symmetries. This represents the interesting case that the lightest hidden sector state is a hidden glueball that can interact with the Standard Model states only via the superheavy fermions. Such states may provide interesting dark matter candidates [23].

In this paper we extended the case studies of realistic free fermionic string models to the case in which the observable universal $S O(10)$ gauge group is broken to the leftright symmetric, $S U(3)_{C} \times S U(2)_{L} \times S U(2)_{R} \times U(1)_{B-L}$, gauge group. We presented three specific examples with this symmetry breaking pattern together with the entire superpotential terms for the first two models, up to quintic order. The distinctive feature of the LRS free fermionic string models, as compared to the previous, FSU5, PS, and SLM cases, is the existence of models which do not contain an anomalous $U(1)$ symmetry. We discussed the general structures which result in the absence, or presence, of an anomalous $U(1)$, in the respective cases. We further contemplated how the string models can motivate the interesting possibility in which the Standard Model fermion spectrum arises from three 16 representations of $S O(10)$, while the weak-hypercharge does not possess the canonical $S O(10)$ embedding. Finally, it will be of further interest to study compactification of other classes of string theories on the manifolds which are associated with the free fermionic models and to examine the properties of the models, which are preserved in these dual constructions. Similarly, 
it is of further interest to study the properties of the LRS string models in relation to the phenomenological studies of LRS field theory models. We shall return to these and related questions in future publications. 


\section{Acknowledgments}

This work is supported in part by DOE Grant No. DE-FG-02-94ER40823 (AEF,CS) and a PPARC advanced fellowship (AEF); and by DOE Grant No. DEFG-0395ER40917 (GBC). 


\section{A Left-Right Symmetric Model 1 Fields}

\begin{tabular}{|c|c|c|c|c|c|c|c|c|c|c|c|c|c|c|}
\hline$F$ & SEC & $(C ; L ; R)$ & $Q_{C}$ & $Q_{1}$ & $Q_{2}$ & $Q_{3}$ & $Q_{4}$ & $Q_{5}$ & $Q_{6}$ & $S U(3)_{H_{1 ; 2}}$ & $Q_{7}$ & $Q_{8}$ & $\overline{Q_{9}}$ & $\overline{Q_{10}}$ \\
\hline$Q_{L_{1}}$ & $\overline{\mathbf{b}_{1}}$ & $(3,2,1)$ & 2 & -2 & $\overline{0}$ & 0 & -2 & 0 & 0 & $(1,1)$ & 0 & 0 & 0 & $\overline{0}$ \\
\hline$Q_{R_{1}}$ & & $(\overline{3}, 1,2)$ & -2 & 2 & 0 & 0 & 2 & 0 & 0 & $(1,1)$ & 0 & 0 & 0 & 0 \\
\hline$L_{L_{1}}$ & & $(1,2,1)$ & -6 & -2 & 0 & 0 & 2 & 0 & 0 & $(1,1)$ & 0 & 0 & 0 & 0 \\
\hline$L_{R_{1}}$ & & $(1,1,2)$ & 6 & 2 & 0 & 0 & -2 & 0 & 0 & $(1,1)$ & 0 & 0 & 0 & 0 \\
\hline$Q_{L_{2}}$ & $\overline{\mathbf{b}_{2}}$ & $(3,2,1)$ & 2 & 0 & -2 & 0 & 0 & -2 & 0 & $(1,1)$ & 0 & 0 & 0 & 0 \\
\hline$Q_{R_{2}}$ & & $(\overline{3}, 1,2)$ & -2 & 0 & 2 & 0 & 0 & 2 & 0 & $(1,1)$ & 0 & 0 & 0 & 0 \\
\hline$L_{L_{2}}$ & & $(1,2,1)$ & -6 & 0 & -2 & 0 & 0 & 2 & 0 & $(1,1)$ & 0 & 0 & 0 & 0 \\
\hline$L_{R_{2}}$ & & $(1,1,2)$ & 6 & 0 & 2 & 0 & 0 & -2 & 0 & $(1,1)$ & 0 & 0 & 0 & 0 \\
\hline$Q_{L_{3}}$ & $\mathbf{b}_{3}$ & $(3,2,1)$ & 2 & 0 & $\overline{0}$ & -2 & 0 & 0 & 2 & $(1,1)$ & 0 & 0 & 0 & $\overline{0}$ \\
\hline$Q_{R_{3}}$ & & $(\overline{3}, 1,2)$ & -2 & 0 & 0 & 2 & 0 & 0 & -2 & $(1,1)$ & 0 & 0 & 0 & 0 \\
\hline$L_{L_{3}}$ & & $(1,2,1)$ & -6 & 0 & 0 & -2 & 0 & 0 & -2 & $(1,1)$ & 0 & 0 & 0 & 0 \\
\hline$L_{R_{3}}$ & & $(1,1,2)$ & 6 & 0 & 0 & 2 & 0 & 0 & 2 & $(1,1)$ & 0 & 0 & 0 & 0 \\
\hline$\overline{\mathcal{L}_{L_{1}}^{+}}$ & $\mathbf{b}_{1}+\beta$ & $(1,2,1)$ & 3 & -1 & 1 & $\overline{1}$ & $\overline{0}$ & 2 & -2 & $(1,1)$ & 0 & -6 & -6 & $\overline{0}$ \\
\hline $\mathcal{L}_{R_{1}}^{+}$ & $\pm \gamma$ & $(1,1,2)$ & 3 & -1 & 1 & 1 & 0 & -2 & 2 & $(1,1)$ & 0 & -6 & -6 & 0 \\
\hline $\mathcal{L}_{L_{1}}^{-}$ & & $(1,2,1)$ & -3 & 1 & -1 & -1 & 0 & 2 & -2 & $(1,1)$ & 0 & 6 & 6 & 0 \\
\hline $\mathcal{L}_{R_{1}}^{-}$ & & $(1,1,2)$ & -3 & 1 & -1 & -1 & 0 & -2 & 2 & $(1,1)$ & 0 & 6 & 6 & 0 \\
\hline$\overline{\mathcal{L}_{L_{2}}^{+}}$ & $\mathbf{b}_{2}+\beta$ & $(1,2,1)$ & 3 & 1 & $\overline{-1}$ & 1 & 2 & 0 & -2 & $(1,1)$ & 0 & -6 & -6 & $\overline{0}$ \\
\hline $\mathcal{L}_{R_{2}}^{+}$ & $\pm \gamma$ & $(1,1,2)$ & 3 & 1 & -1 & 1 & -2 & 0 & 2 & $(1,1)$ & 0 & -6 & -6 & 0 \\
\hline $\mathcal{L}_{L_{2}}^{-}$ & & $(1,2,1)$ & -3 & -1 & 1 & -1 & 2 & 0 & -2 & $(1,1)$ & 0 & 6 & 6 & 0 \\
\hline $\mathcal{L}_{R_{2}}^{-}$ & & $(1,1,2)$ & -3 & -1 & 1 & -1 & -2 & 0 & 2 & $(1,1)$ & 0 & 6 & 6 & 0 \\
\hline $\mathcal{L}_{L_{3}}^{+}$ & $\mathbf{b}_{3}+\beta$ & $(1,2,1)$ & 3 & 1 & 1 & -1 & 2 & 2 & 0 & $(1,1)$ & 0 & -6 & -6 & 0 \\
\hline $\mathcal{L}_{R_{3}}^{+}$ & $\pm \gamma$ & $(1,1,2)$ & 3 & 1 & 1 & -1 & -2 & -2 & 0 & $(1,1)$ & 0 & -6 & -6 & 0 \\
\hline $\mathcal{L}_{L_{3}}^{-}$ & & $(1,2,1)$ & -3 & -1 & -1 & 1 & 2 & 2 & 0 & $(1,1)$ & 0 & 6 & 6 & 0 \\
\hline $\mathcal{L}_{R_{3}}^{-}$ & & $(1,1,2)$ & -3 & -1 & -1 & 1 & -2 & -2 & 0 & $(1,1)$ & 0 & 6 & 6 & 0 \\
\hline $\mathcal{L}_{L_{12}}^{+}$ & $\mathbf{1}+\mathbf{b}_{1}+$ & $(1,2,1)$ & 0 & 2 & $\overline{2}$ & 0 & $\overline{0}$ & 0 & 2 & $(1,1)$ & 4 & 0 & 0 & 12 \\
\hline $\mathcal{L}_{R_{12}}^{+}$ & $\mathbf{b}_{2}+2 \gamma$ & $(1,1,2)$ & 0 & -2 & -2 & 0 & 0 & 0 & -2 & $(1,1)$ & 4 & 0 & 0 & 12 \\
\hline $\mathcal{L}_{L_{12}}^{-}$ & & $(1,2,1)$ & 0 & 2 & 2 & 0 & 0 & 0 & 2 & $(1,1)$ & -4 & 0 & 0 & -12 \\
\hline $\mathcal{L}_{R_{12}}^{-}$ & & $(1,1,2)$ & 0 & -2 & -2 & 0 & 0 & 0 & -2 & $(1,1)$ & -4 & 0 & 0 & -12 \\
\hline $\mathcal{L}_{L_{13}}^{+}$ & $\mathbf{1}+\mathbf{b}_{1}+$ & $(1,2,1)$ & 0 & 2 & 0 & 2 & $\overline{0}$ & -2 & 0 & $(1,1)$ & 4 & 0 & 0 & 12 \\
\hline $\mathcal{L}_{R_{13}}^{+}$ & $\mathbf{b}_{3}+2 \gamma$ & $(1,1,2)$ & 0 & -2 & 0 & -2 & 0 & 2 & 0 & $(1,1)$ & 4 & 0 & 0 & 12 \\
\hline $\mathcal{L}_{L_{13}}^{-}$ & & $(1,2,1)$ & 0 & 2 & 0 & 2 & 0 & -2 & 0 & $(1,1)$ & -4 & 0 & 0 & -12 \\
\hline $\mathcal{L}_{R_{13}}^{-}$ & & $(1,1,2)$ & 0 & -2 & 0 & -2 & 0 & 2 & 0 & $(1,1)$ & -4 & 0 & 0 & -12 \\
\hline
\end{tabular}

Table 1: Model 1 fields. Charges have been multiplied by 4 . 


\begin{tabular}{|c|c|c|c|c|c|c|c|c|c|c|c|c|c|c|}
\hline$F$ & SEC & $(C ; L ; R)$ & $Q_{C}$ & $Q_{1}$ & $Q_{2}$ & $Q_{3}$ & $Q_{4}$ & $Q_{5}$ & $\overline{Q_{6}}$ & $S U(3)_{H_{1 ; 2}}$ & $Q_{7}$ & $Q_{8}$ & $Q_{9}$ & $Q_{10}$ \\
\hline $\mathcal{L}_{L_{23}}^{+}$ & $\mathbf{1}+\mathbf{b}_{2}+$ & $(1,2,1)$ & 0 & 0 & 2 & 2 & -2 & 0 & 0 & $(1,1)$ & 4 & 0 & 0 & 12 \\
\hline $\mathcal{L}_{R_{23}}^{+}$ & $\mathbf{b}_{3}+2 \gamma$ & $(1,1,2)$ & 0 & 0 & -2 & -2 & 2 & 0 & 0 & $(1,1)$ & 4 & 0 & 0 & 12 \\
\hline $\mathcal{L}_{L_{23}}^{-20}$ & & $(1,1,2)$ & 0 & 0 & 2 & 2 & -2 & 0 & 0 & $(1,1)$ & -4 & 0 & 0 & -12 \\
\hline $\mathcal{L}_{R_{23}}^{-23}$ & & $(1,2,1)$ & 0 & 0 & -2 & -2 & 2 & 0 & 0 & $(1,1)$ & -4 & 0 & 0 & -12 \\
\hline$D_{\alpha \beta}$ & $\mathbf{S}+\beta$ & $(3,1,1)$ & -1 & -1 & -1 & -1 & 2 & 2 & 2 & $(1,1)$ & 0 & 6 & 6 & 0 \\
\hline $\bar{D}_{\alpha \beta}$ & $\pm \gamma$ & $(\overline{3}, 1,1)$ & 1 & 1 & 1 & 1 & -2 & -2 & -2 & $(1,1)$ & 0 & -6 & -6 & 0 \\
\hline$h_{1}$ & Neveu- & $(1,2,2)$ & 0 & 0 & 0 & 0 & 0 & 0 & 0 & $(1,1)$ & 0 & 0 & 0 & 0 \\
\hline$h_{2}$ & Schwarz & $(1,2,2)$ & 0 & 0 & 0 & 0 & 0 & 0 & 0 & $(1,1)$ & 0 & 0 & 0 & 0 \\
\hline$\Phi_{1}$ & & $(1,1,1)$ & 0 & 0 & 0 & 0 & 0 & 0 & 0 & $(1,1)$ & 0 & 0 & 0 & 0 \\
\hline$\Phi_{2}$ & & $(1,1,1)$ & 0 & 0 & 0 & 0 & 0 & 0 & 0 & $(1,1)$ & 0 & 0 & 0 & 0 \\
\hline$\Phi_{3}$ & & $(1,1,1)$ & 0 & 0 & 0 & 0 & 0 & 0 & 0 & $(1,1)$ & 0 & 0 & 0 & 0 \\
\hline$\Phi_{12}$ & & $(1,1,1)$ & 0 & -4 & 4 & 0 & 0 & 0 & 0 & $(1,1)$ & 0 & 0 & 0 & 0 \\
\hline $\bar{\Phi}_{12}$ & & $(1,1,1)$ & 0 & 4 & -4 & 0 & 0 & 0 & 0 & $(1,1)$ & 0 & 0 & 0 & 0 \\
\hline$\Phi_{23}$ & & $(1,1,1)$ & 0 & 0 & -4 & 4 & 0 & 0 & 0 & $(1,1)$ & 0 & 0 & 0 & 0 \\
\hline $\bar{\Phi}_{23}$ & & $(1,1,1)$ & 0 & 0 & 4 & -4 & 0 & 0 & 0 & $(1,1)$ & 0 & 0 & 0 & 0 \\
\hline$\Phi_{31}$ & & $(1,1,1)$ & 0 & -4 & 0 & 4 & 0 & 0 & 0 & $(1,1)$ & 0 & 0 & 0 & 0 \\
\hline $\bar{\Phi}_{31}$ & & $(1,1,1)$ & 0 & 4 & 0 & -4 & 0 & 0 & 0 & $(1,1)$ & 0 & 0 & 0 & 0 \\
\hline$\phi_{1}$ & $\mathbf{1}+\mathbf{S}+\mathbf{b}_{3}+$ & $(1,1,1)$ & 0 & 2 & -2 & 0 & 0 & 0 & 0 & $(1,1)$ & 4 & 12 & -12 & 4 \\
\hline $\bar{\phi}_{1}$ & $\alpha+\beta$ & $(1,1,1)$ & 0 & -2 & 2 & 0 & 0 & 0 & 0 & $(1,1)$ & -4 & -12 & 12 & -4 \\
\hline$\phi_{2}$ & & $(1,1,1)$ & 0 & 2 & -2 & 0 & 0 & 0 & 0 & $(1,1)$ & 4 & 12 & 12 & -4 \\
\hline $\bar{\phi}_{2}$ & & $(1,1,1)$ & 0 & -2 & 2 & 0 & 0 & 0 & 0 & $(1,1)$ & -4 & -12 & -12 & 4 \\
\hline$\phi_{3}$ & & $(1,1,1)$ & 0 & 2 & -2 & 0 & 0 & 0 & 0 & $(1,1)$ & -4 & -12 & -12 & 4 \\
\hline $\bar{\phi}_{3}$ & & $(1,1,1)$ & 0 & -2 & 2 & 0 & 0 & 0 & 0 & $(1,1)$ & 4 & 12 & 12 & -4 \\
\hline$\phi_{4}$ & & $(1,1,1)$ & 0 & 2 & -2 & 0 & 0 & 0 & 0 & $(1,1)$ & -4 & -12 & 12 & -4 \\
\hline $\bar{\phi}_{4}$ & & $(1,1,1)$ & 0 & -2 & 2 & 0 & 0 & 0 & 0 & $(1,1)$ & 4 & 12 & -12 & 4 \\
\hline$\phi_{5}$ & $\overline{\mathbf{S}+\beta}$ & $(1,1,1)$ & -3 & -3 & 1 & 1 & 2 & 2 & -2 & $(1,1)$ & 0 & -6 & -6 & 0 \\
\hline $\bar{\phi}_{5}$ & $\pm \gamma$ & $(1,1,1)$ & 3 & 3 & -1 & -1 & -2 & -2 & 2 & $(1,1)$ & 0 & 6 & 6 & 0 \\
\hline$\phi_{6}$ & & $(1,1,1)$ & -3 & 1 & -3 & 1 & 2 & 2 & -2 & $(1,1)$ & 0 & -6 & -6 & 0 \\
\hline $\bar{\phi}_{6}$ & & $(1,1,1)$ & 3 & -1 & 3 & -1 & -2 & -2 & 2 & $(1,1)$ & 0 & 6 & 6 & 0 \\
\hline$\phi_{7}$ & & $(1,1,1)$ & -3 & 1 & 1 & -3 & 2 & 2 & -2 & $(1,1)$ & 0 & -6 & -6 & 0 \\
\hline $\bar{\phi}_{7}$ & & $(1,1,1)$ & 3 & -1 & -1 & 3 & -2 & -2 & 2 & $(1,1)$ & 0 & 6 & 6 & 0 \\
\hline
\end{tabular}

Table 1 continued: Model 1 fields. 


\begin{tabular}{|c|c|c|rrrrrrr|r|rrrr|}
\hline$F$ & SEC & $(C ; L ; R)$ & $Q_{C}$ & $Q_{1}$ & $Q_{2}$ & $Q_{3}$ & $Q_{4}$ & $Q_{5}$ & $Q_{6}$ & $S U(3)_{H_{1 ;}}$ & $Q_{7}$ & $Q_{8}$ & $Q_{9}$ & $Q_{10}$ \\
\hline$\phi_{8}$ & $\mathbf{1}+\mathbf{b}_{1}+$ & $(1,1,1)$ & -3 & 1 & 1 & 1 & -2 & 2 & -2 & $(1,1)$ & 4 & 6 & 6 & 12 \\
$\bar{\phi}_{8}$ & $\mathbf{b}_{2}+\mathbf{b}_{3}+$ & $(1,1,1)$ & 3 & -1 & -1 & -1 & 2 & -2 & 2 & $(1,1)$ & -4 & -6 & -6 & -12 \\
$\phi_{9}$ & $\beta \pm \gamma$ & $(1,1,1)$ & -3 & 1 & 1 & 1 & 2 & -2 & -2 & $(1,1)$ & -4 & 6 & 6 & -12 \\
$\bar{\phi}_{9}$ & & $(1,1,1)$ & 3 & -1 & -1 & -1 & -2 & 2 & 2 & $(1,1)$ & 4 & -6 & -6 & 12 \\
\hline$\phi_{10}$ & $\mathbf{S}+\mathbf{b}_{1}+$ & $(1,1,1)$ & 6 & 0 & 0 & -2 & 0 & 0 & 0 & $(1,1)$ & 0 & 4 & -12 & 0 \\
$\bar{\phi}_{10}$ & $\mathbf{b}_{2}+\alpha+$ & $(1,1,1)$ & -6 & 0 & 0 & 2 & 0 & 0 & 0 & $(1,1)$ & 0 & -4 & 12 & 0 \\
$\phi_{11}$ & $\beta+2 \gamma$ & $(1,1,1)$ & 6 & 0 & 0 & -2 & 0 & 0 & 0 & $(1,1)$ & 0 & -4 & -12 & 0 \\
$\bar{\phi}_{11}$ & & $(1,1,1)$ & -6 & 0 & 0 & 2 & 0 & 0 & 0 & $(1,1)$ & 0 & 4 & 12 & 0 \\
\hline$H_{1}$ & $\mathbf{S}+\mathbf{b}_{1}$ & $(1,1,1)$ & 0 & -2 & -2 & 0 & 0 & 0 & 0 & $(3,1)$ & -4 & -4 & -4 & 4 \\
$\bar{H}_{1}$ & $+\mathbf{b}_{2}+\alpha$ & $(1,1,1)$ & 0 & 2 & 2 & 0 & 0 & 0 & 0 & $(\overline{3}, 1)$ & 4 & 4 & 4 & -4 \\
$H_{2}$ & $+\beta$ & $(1,1,1)$ & 0 & 2 & 2 & 0 & 0 & 0 & 0 & $(1,3)$ & -4 & 4 & 4 & 4 \\
$\bar{H}_{2}$ & $\oplus \zeta$ & $(1,1,1)$ & 0 & -2 & -2 & 0 & 0 & 0 & 0 & $(1, \overline{3})$ & 4 & -4 & -4 & -4 \\
\hline
\end{tabular}

Table 1 continued: Model 1 fields. 


\section{B Left-Right Symmetric Model 1 Superpotential Terms}

Model 1 Fifth Order Superpotential:

$W_{5}$ (observable):

\begin{tabular}{|c|c|c|c|}
\hline & 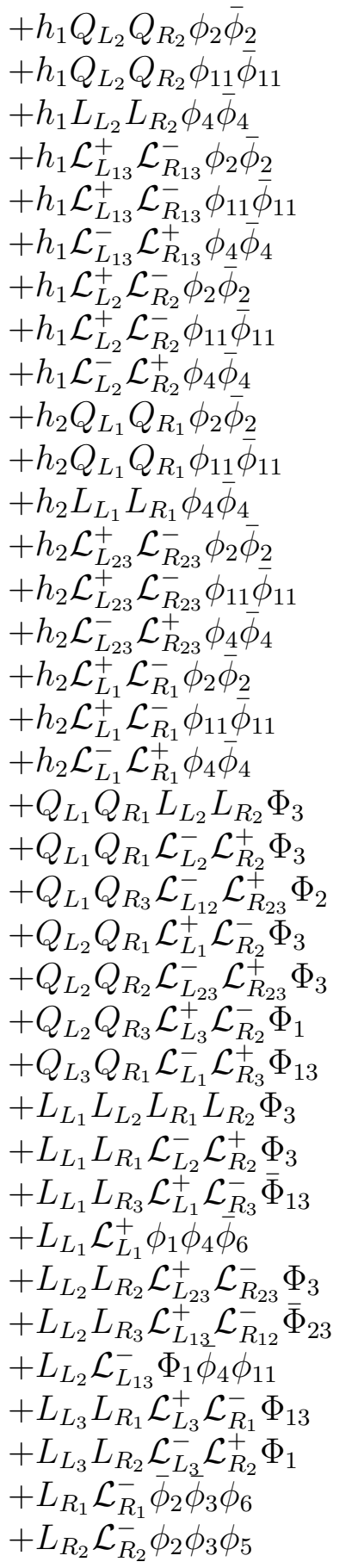 & 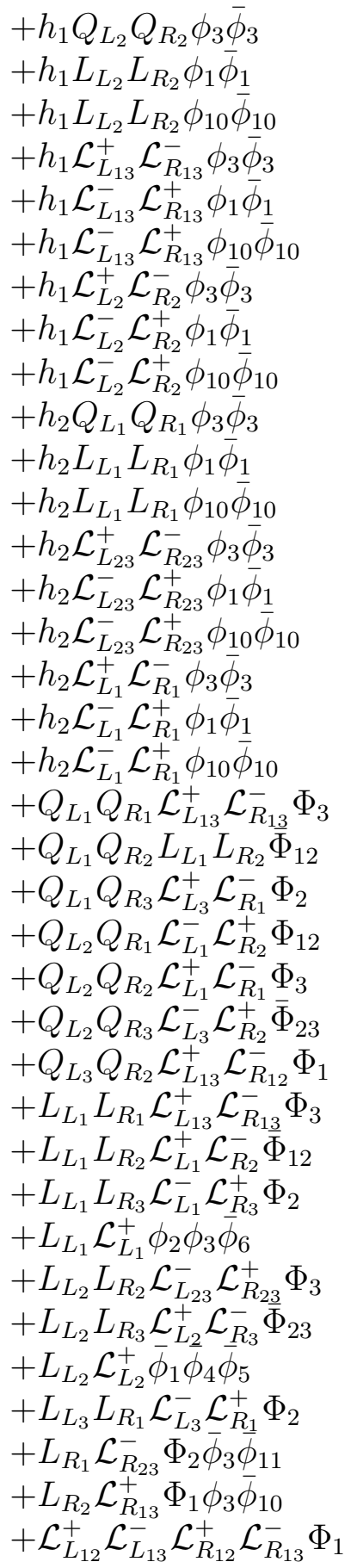 & 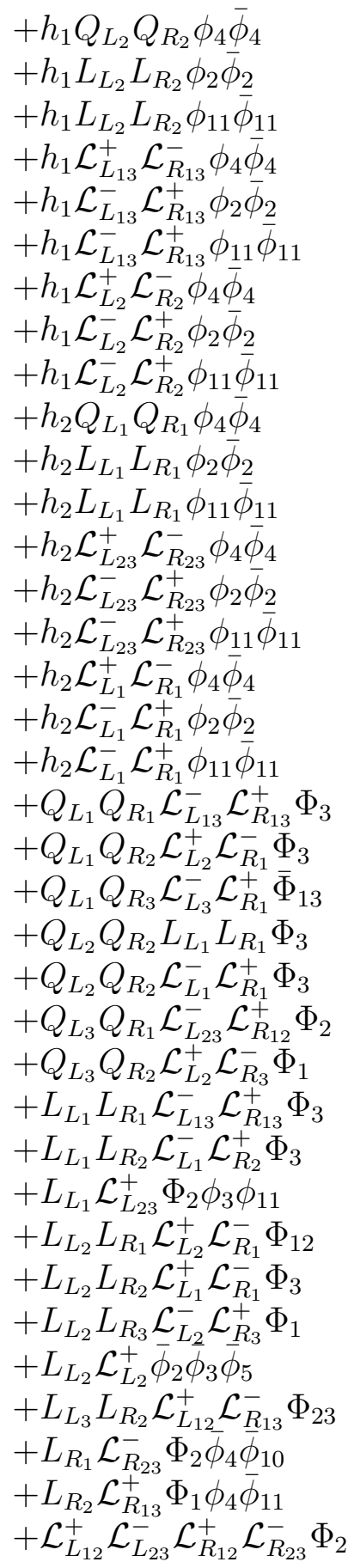 \\
\hline
\end{tabular}


$W_{5}$ (observable) continued:

$$
\begin{aligned}
& +\mathcal{L}_{L_{12}}^{+} \mathcal{L}_{L_{1}}^{+} \mathcal{L}_{R_{23}}^{-} \mathcal{L}_{R_{3}}^{-} \Phi_{2} \quad+\mathcal{L}_{L_{12}}^{+} \mathcal{L}_{L_{1}}^{-} \mathcal{L}_{R_{23}}^{-} \mathcal{L}_{R_{3}}^{+} \Phi_{13} \quad+\mathcal{L}_{L_{12}}^{-} \mathcal{L}_{L_{13}}^{+} \mathcal{L}_{R_{12}}^{-} \mathcal{L}_{R_{13}}^{+} \Phi_{1} \quad+\mathcal{L}_{L_{12}}^{-} \mathcal{L}_{L_{23}}^{+} \mathcal{L}_{R_{12}}^{-} \mathcal{L}_{R_{23}}^{+} \Phi_{2} \\
& +\mathcal{L}_{L_{12}}^{-} \mathcal{L}_{L_{2}}^{+} \mathcal{L}_{R_{13}}^{+} \mathcal{L}_{R_{3}}^{-} \Phi_{1} \quad+\mathcal{L}_{L_{12}}^{-} \mathcal{L}_{L_{2}}^{-} \mathcal{L}_{R_{13}}^{+} \mathcal{L}_{R_{3}}^{+} \Phi_{23} \quad+\mathcal{L}_{L_{13}}^{+} \mathcal{L}_{L_{23}}^{+} \mathcal{L}_{R_{13}}^{-} \mathcal{L}_{R_{23}}^{-} \Phi_{3} \quad+\mathcal{L}_{L_{13}}^{+} \mathcal{L}_{L_{23}}^{-} \mathcal{L}_{R_{13}}^{+} \mathcal{L}_{R_{23}}^{-} \Phi_{3} \\
& +\mathcal{L}_{L_{13}}^{+} \mathcal{L}_{L_{23}}^{-} \mathcal{L}_{R_{13}}^{-} \mathcal{L}_{R_{23}}^{+} \Phi_{3} \quad+\mathcal{L}_{L_{13}}^{+} \mathcal{L}_{L_{1}}^{+} \mathcal{L}_{R_{13}}^{-} \mathcal{L}_{R_{1}}^{-} \Phi_{3} \quad+\mathcal{L}_{L_{13}}^{+} \mathcal{L}_{L_{1}}^{-} \mathcal{L}_{R_{13}}^{-} \mathcal{L}_{R_{1}}^{+} \Phi_{3} \quad+\mathcal{L}_{L_{13}}^{-} \mathcal{L}_{L_{23}}^{+} \mathcal{L}_{R_{13}}^{+} \mathcal{L}_{R_{23}}^{-} \Phi_{3} \\
& +\mathcal{L}_{L_{13}}^{-} \mathcal{L}_{L_{23}}^{+} \mathcal{L}_{R_{13}}^{-} \mathcal{L}_{R_{23}}^{+} \Phi_{3} \quad+\mathcal{L}_{L_{13}}^{-} \mathcal{L}_{L_{23}}^{-} \mathcal{L}_{R_{13}}^{+} \mathcal{L}_{R_{23}}^{+} \Phi_{3} \quad+\mathcal{L}_{L_{13}}^{-} \mathcal{L}_{L_{1}}^{+} \mathcal{L}_{R_{13}}^{+} \mathcal{L}_{R_{1}}^{-} \Phi_{3} \quad+\mathcal{L}_{L_{13}}^{-} \mathcal{L}_{L_{1}}^{-} \mathcal{L}_{R_{13}}^{+} \mathcal{L}_{R_{1}}^{+} \Phi_{3} \\
& +\mathcal{L}_{L_{13}}^{-} \mathcal{L}_{L_{3}}^{+} \mathcal{L}_{R_{12}}^{+} \mathcal{L}_{R_{2}}^{-} \Phi_{1} \quad+\mathcal{L}_{L_{13}}^{-} \mathcal{L}_{L_{3}}^{-} \mathcal{L}_{R_{12}}^{+} \mathcal{L}_{R_{2}}^{+} \Phi_{23} \quad+\mathcal{L}_{L_{23}}^{+} \mathcal{L}_{L_{2}}^{+} \mathcal{L}_{R_{23}}^{-} \mathcal{L}_{R_{2}}^{-} \Phi_{3} \quad+\mathcal{L}_{L_{23}}^{+} \mathcal{L}_{L_{2}}^{-} \mathcal{L}_{R_{23}}^{-} \mathcal{L}_{R_{2}}^{+} \Phi_{3} \\
& +\mathcal{L}_{L_{23}}^{+} \mathcal{L}_{L_{3}}^{+} \mathcal{L}_{R_{12}}^{-} \mathcal{L}_{R_{1}}^{-} \Phi_{2} \quad+\mathcal{L}_{L_{23}}^{+} \mathcal{L}_{L_{3}}^{-} \mathcal{L}_{R_{12}}^{-} \mathcal{L}_{R_{1}}^{+} \bar{\Phi}_{13} \quad+\mathcal{L}_{L_{23}}^{-} \mathcal{L}_{L_{2}}^{+} \mathcal{L}_{R_{23}}^{+} \mathcal{L}_{R_{2}}^{-} \Phi_{3} \quad+\mathcal{L}_{L_{23}}^{-} \mathcal{L}_{L_{2}}^{-} \mathcal{L}_{R_{23}}^{+} \mathcal{L}_{R_{2}}^{+} \Phi_{3} \\
& +\mathcal{L}_{L_{1}}^{+} \mathcal{L}_{L_{2}}^{+} \mathcal{L}_{R_{1}}^{-} \mathcal{L}_{R_{2}}^{-} \Phi_{3} \quad+\mathcal{L}_{L_{1}}^{+} \mathcal{L}_{L_{2}}^{-} \mathcal{L}_{R_{1}}^{+} \mathcal{L}_{R_{2}}^{-} \Phi_{12} \quad+\mathcal{L}_{L_{1}}^{+} \mathcal{L}_{L_{2}}^{-} \mathcal{L}_{R_{1}}^{-} \mathcal{L}_{R_{2}}^{+} \Phi_{3} \quad+\mathcal{L}_{L_{1}}^{-} \mathcal{L}_{L_{2}}^{+} \mathcal{L}_{R_{1}}^{+} \mathcal{L}_{R_{2}}^{-} \Phi_{3} \\
& +\mathcal{L}_{L_{1}}^{-} \mathcal{L}_{L_{2}}^{+} \mathcal{L}_{R_{1}}^{-} \mathcal{L}_{R_{2}}^{+} \Phi_{12} \quad+\mathcal{L}_{L_{1}}^{-} \mathcal{L}_{L_{2}}^{-} \mathcal{L}_{R_{1}}^{+} \mathcal{L}_{R_{2}}^{+} \Phi_{3}
\end{aligned}
$$

$W_{5}$ (mixed):

$$
\begin{array}{lllll} 
& h_{1} Q_{L_{2}} Q_{R_{2}} H_{1} \bar{H}_{1} & +h_{1} Q_{L_{2}} Q_{R_{2}} H_{2} \bar{H}_{2} & +h_{1} L_{L_{2}} L_{R_{2}} H_{1} \bar{H}_{1} & +h_{1} L_{L_{2}} L_{R_{2}} H_{2} \bar{H}_{2} \\
+h_{1} \mathcal{L}_{L_{13}}^{+} \mathcal{L}_{R_{13}}^{-} H_{1} \bar{H}_{1} & +h_{1} \mathcal{L}_{L_{13}}^{+} \mathcal{L}_{R_{13}}^{-} H_{2} \bar{H}_{2} & +h_{1} \mathcal{L}_{L_{13}}^{-} \mathcal{L}_{R_{13}}^{+} H_{1} \bar{H}_{1} & +h_{1} \mathcal{L}_{L_{13}}^{-} \mathcal{L}_{R_{13}}^{+} H_{2} \bar{H}_{2} \\
+h_{1} \mathcal{L}_{L_{2}}^{+} \mathcal{L}_{R_{2}}^{-} H_{1} \bar{H}_{1} & +h_{1} \mathcal{L}_{L_{2}}^{+} \mathcal{L}_{R_{2}}^{-} H_{2} \bar{H}_{2} & +h_{1} \mathcal{L}_{L_{2}}^{-} \mathcal{L}_{R_{2}}^{+} H_{1} \bar{H}_{1} & +h_{1} \mathcal{L}_{L_{2}}^{-} \mathcal{L}_{R_{2}}^{+} H_{2} \bar{H}_{2} \\
+h_{2} Q_{L_{1}} Q_{R_{1}} H_{1} \bar{H}_{1} & +h_{2} Q_{L_{1}} Q_{R_{1}} H_{2} \bar{H}_{2} & +h_{2} L_{L_{1}} L_{R_{1}} H_{1} \bar{H}_{1} & +h_{2} L_{L_{1}} L_{R_{1}} H_{2} \bar{H}_{2} \\
+h_{2} \mathcal{L}_{L_{23}}^{+} \mathcal{L}_{R_{23}}^{-} H_{1} \bar{H}_{1} & +h_{2} \mathcal{L}_{L_{23}}^{+} \mathcal{L}_{R_{23}}^{-} H_{2} \bar{H}_{2} & +h_{2} \mathcal{L}_{L_{23}}^{-} \mathcal{L}_{R_{23}}^{+} H_{1} \bar{H}_{1} & +h_{2} \mathcal{L}_{L_{23}}^{-} \mathcal{L}_{R_{23}}^{+} H_{2} \bar{H}_{2} \\
+h_{2} \mathcal{L}_{L_{1}}^{+} \mathcal{L}_{R_{1}}^{-} H_{1} \bar{H}_{1} & +h_{2} \mathcal{L}_{L_{1}}^{+} \mathcal{L}_{R_{1}}^{-} H_{2} \bar{H}_{2} & +h_{2} \mathcal{L}_{L_{1}}^{-} \mathcal{L}_{R_{1}}^{+} H_{1} \bar{H}_{1} & +h_{2} \mathcal{L}_{L_{1}}^{-} \mathcal{L}_{R_{1}}^{+} H_{2} \bar{H}_{2}
\end{array}
$$

$W_{5}($ singlets $), W_{5}($ hidden $)$ : none 


\section{Left-Right Symmetric Model 2 Fields}

\begin{tabular}{|c|c|c|rrrrrr|r|rr|}
\hline$F$ & SEC & $S U(3)_{C} \times S U(2)_{L ; R ; C}$ & $Q_{1^{\prime}}$ & $Q_{2^{\prime}}$ & $Q_{3^{\prime}}$ & $Q_{4}$ & $Q_{5}$ & $Q_{6}$ & $S U(4)_{H_{1 ; 2}}$ & $Q_{7^{\prime \prime}}$ & $Q_{8^{\prime \prime}}$ \\
\hline$Q_{L_{1}}$ & $\mathbf{b}_{1} \oplus$ & $(3,2,1,1)$ & -2 & -2 & -4 & -2 & 0 & 0 & $(1,1)$ & 0 & -16 \\
$Q_{R_{1}}$ & $\mathbf{b}_{1}+\zeta+2 \gamma$ & $(\overline{3}, 1,2,1)$ & 2 & 2 & 4 & 2 & 0 & 0 & $(1,1)$ & 0 & 16 \\
$L_{L_{1}}$ & & $(1,2,1,2)$ & -2 & -2 & 4 & 2 & 0 & 0 & $(1,1)$ & -8 & 16 \\
$L_{R_{1}}$ & & $(1,1,2,2)$ & 2 & 2 & -4 & -2 & 0 & 0 & $(1,1)$ & 8 & -16 \\
$S_{L_{1}}$ & & $(1,2,1,1)$ & -2 & -2 & 4 & 2 & 0 & 0 & $(1,1)$ & 16 & 16 \\
$S_{R_{1}}$ & & $(1,1,2,1)$ & 2 & 2 & -4 & -2 & 0 & 0 & $(1,1)$ & -16 & -16 \\
\hline$Q_{L_{2}}$ & $\mathbf{b}_{2} \oplus$ & $(3,2,1,1)$ & 2 & -2 & -4 & 0 & -2 & 0 & $(1,1)$ & 0 & -16 \\
$Q_{R_{2}}$ & $\mathbf{b}_{2}+\zeta+2 \gamma$ & $(\overline{3}, 1,2,1)$ & -2 & 2 & 4 & 0 & 2 & 0 & $(1,1)$ & 0 & 16 \\
$L_{L_{2}}$ & & $(1,2,1,2)$ & 2 & -2 & 4 & 0 & 2 & 0 & $(1,1)$ & -8 & 16 \\
$L_{R_{2}}$ & & $(1,1,2,2)$ & -2 & 2 & -4 & 0 & -2 & 0 & $(1,1)$ & 8 & -16 \\
$S_{L_{2}}$ & & $(1,2,1,1)$ & 2 & -2 & 4 & 0 & 2 & 0 & $(1,1)$ & 16 & 16 \\
$S_{R_{2}}$ & & $(1,1,2,1)$ & -2 & 2 & -4 & 0 & -2 & 0 & $(1,1)$ & -16 & -16 \\
\hline$Q_{L_{3}}$ & $\mathbf{b}_{3} \oplus$ & $(3,2,1,1)$ & 0 & 4 & -4 & 0 & 0 & -2 & $(1,1)$ & 0 & -16 \\
$Q_{R_{3}}$ & $\mathbf{b}_{3}+\zeta+2 \gamma$ & $(3,1,2,1)$ & 0 & -4 & 4 & 0 & 0 & 2 & $(1,1)$ & 0 & 16 \\
$L_{L_{3}}$ & & $(1,2,1,2)$ & 0 & 4 & 4 & 0 & 0 & 2 & $(1,1)$ & -8 & 16 \\
$L_{R_{3}}$ & & $(1,1,2,2)$ & 0 & -4 & -4 & 0 & 0 & -2 & $(1,1)$ & 8 & -16 \\
$S_{L_{3}}$ & & $(1,2,1,1)$ & 0 & 4 & 4 & 0 & 0 & 2 & $(1,1)$ & 16 & 16 \\
$S_{R_{3}}$ & & $(1,1,2,1)$ & 0 & -4 & -4 & 0 & 0 & -2 & $(1,1)$ & -16 & -16 \\
\hline $\mathcal{L}_{L_{1}}$ & $\mathbf{b}_{1}+$ & $(1,2,1,1)$ & -2 & -2 & 12 & 0 & 0 & 0 & $(1,1)$ & -8 & -8 \\
$\overline{\mathcal{L}}_{L_{1}}$ & $\alpha+\beta \pm \gamma$ & $(1,2,1,1)$ & 2 & 2 & -12 & 0 & 0 & 0 & $(1,1)$ & 8 & 8 \\
$\mathcal{L}_{R_{1}}$ & & $(1,1,2,1)$ & -2 & -2 & 12 & 0 & 0 & 0 & $(1,1)$ & -8 & -8 \\
$\overline{\mathcal{L}}_{R_{1}}$ & & $(1,1,2,1)$ & 2 & 2 & -12 & 0 & 0 & 0 & $(1,1)$ & 8 & 8 \\
\hline $\mathcal{L}_{L_{2}}$ & $\mathbf{1}+\mathbf{b}_{1}+$ & $(1,2,1,1)$ & 2 & -2 & -4 & 0 & 0 & 0 & $(1,1)$ & -8 & 40 \\
$\overline{\mathcal{L}}_{L_{2}}$ & $\mathbf{b}_{3} \pm \gamma$ & $(1,2,1,1)$ & -2 & 2 & 4 & 0 & 0 & 0 & $(1,1)$ & 8 & -40 \\
$\mathcal{L}_{R_{2}}$ & & $(1,1,2,1)$ & 2 & -2 & -4 & 0 & 0 & 0 & $(1,1)$ & -8 & 40 \\
$\overline{\mathcal{L}}_{R_{2}}$ & & $(1,1,2,1)$ & -2 & 2 & 4 & 0 & 0 & 0 & $(1,1)$ & 8 & -40 \\
\hline
\end{tabular}

Table 2: Model 2 fields. Charges have been multiplied by 4 . 


\begin{tabular}{|c|c|c|c|c|c|c|c|c|c|c|c|}
\hline$F$ & SEC & $S U(3)_{C} \times S U(2)_{L ; R ; C}$ & $Q_{1^{\prime}}$ & $Q_{2^{\prime}}$ & $Q_{3^{\prime}}$ & $Q_{4}$ & $Q_{5}$ & $Q_{6}$ & $S U(4)_{H_{1 ; 2}}$ & $Q_{7^{\prime \prime}}$ & $Q_{8^{\prime \prime}}$ \\
\hline$D_{1}$ & $I+S+2 \gamma$ & $(3,1,1,1)$ & -4 & -4 & 0 & 0 & 0 & 0 & $(1,1)$ & 16 & 0 \\
\hline $\bar{D}_{1}$ & & $(\overline{3}, 1,1,1)$ & 4 & 4 & 0 & 0 & 0 & 0 & $(1,1)$ & -16 & 0 \\
\hline$D_{2}$ & & $(3,1,1,1)$ & 4 & -4 & 0 & 0 & 0 & 0 & $(1,1)$ & 16 & 0 \\
\hline $\bar{D}_{2}$ & & $(\overline{3}, 1,1,1)$ & -4 & 4 & 0 & 0 & 0 & 0 & $(1,1)$ & -16 & 0 \\
\hline$D_{3}$ & & $(3,1,1,1)$ & 0 & 8 & 0 & 0 & 0 & 0 & $(1,1)$ & 16 & 0 \\
\hline $\bar{D}_{3}$ & & $(\overline{3}, 1,1,1)$ & 0 & -8 & 0 & 0 & 0 & 0 & $(1,1)$ & -16 & 0 \\
\hline$\overline{D_{\alpha \beta}}$ & $\xi=S+$ & $(3,1,1,1)$ & 0 & 4 & 8 & 0 & 0 & 0 & $(1,1)$ & 0 & 32 \\
\hline $\bar{D}_{\alpha \beta}$ & $\mathbf{b}_{1}+\mathbf{b}_{2}+$ & $(\overline{3}, 1,1,1)$ & 0 & -4 & -8 & 0 & 0 & 0 & $(1,1)$ & 0 & -32 \\
\hline$N_{\alpha \beta}$ & $\alpha+\beta$ & $(1,1,1,2)$ & 0 & 4 & -8 & 0 & 0 & 0 & $(1,1)$ & -8 & -32 \\
\hline $\bar{N}_{\alpha \beta}$ & & $(1,1,1,2)$ & 0 & -4 & 8 & 0 & 0 & 0 & $(1,1)$ & 8 & 32 \\
\hline$\phi_{1}^{ \pm}$ & $\oplus \xi+\zeta+2 \gamma$ & $(1,1,1,1)$ & 4 & 0 & 0 & \pm 4 & 0 & 0 & $(1,1)$ & 0 & 0 \\
\hline $\bar{\phi}_{1}^{ \pm}$ & & $(1,1,1,1)$ & -4 & 0 & 0 & $\mp 4$ & 0 & 0 & $(1,1)$ & 0 & 0 \\
\hline$\phi_{2}^{ \pm}$ & & $(1,1,1,1)$ & 4 & 0 & 0 & 0 & \pm 4 & 0 & $(1,1)$ & 0 & 0 \\
\hline $\bar{\phi}_{2}^{ \pm}$ & & $(1,1,1,1)$ & -4 & 0 & 0 & 0 & $\mp 4$ & 0 & $(1,1)$ & 0 & 0 \\
\hline$\phi_{3}^{ \pm}$ & & $(1,1,1,1)$ & 4 & 0 & 0 & 0 & 0 & \pm 4 & $(1,1)$ & 0 & 0 \\
\hline $\bar{\phi}_{3}^{ \pm}$ & & $(1,1,1,1)$ & -4 & 0 & 0 & 0 & 0 & $\mp 4$ & $(1,1)$ & 0 & 0 \\
\hline$\phi_{4}$ & & $(1,1,1,1)$ & 0 & 4 & -8 & 0 & 0 & 0 & $(1,1)$ & 16 & -32 \\
\hline $\bar{\phi}_{4}$ & & $(1,1,1,1)$ & 0 & -4 & 8 & 0 & 0 & 0 & $(1,1)$ & -16 & 32 \\
\hline$h_{1}$ & Neveu- & $(1,2,2,1)$ & 0 & 0 & 0 & 0 & 0 & 0 & $(1,1)$ & 0 & 0 \\
\hline$h_{2}$ & Schwarz & $(1,2,2,1)$ & 0 & 0 & 0 & 0 & 0 & 0 & $(1,1)$ & 0 & 0 \\
\hline$\Phi_{1}$ & & $(1,1,1,1)$ & 0 & 0 & 0 & 0 & 0 & 0 & $(1,1)$ & 0 & 0 \\
\hline$\Phi_{2}$ & & $(1,1,1,1)$ & 0 & 0 & 0 & 0 & 0 & 0 & $(1,1)$ & 0 & 0 \\
\hline$\Phi_{3}$ & & $(1,1,1,1)$ & 0 & 0 & 0 & 0 & 0 & 0 & $(1,1)$ & 0 & 0 \\
\hline$\Phi_{12}$ & & $(1,1,1,1)$ & -8 & 0 & 0 & 0 & 0 & 0 & $(1,1)$ & 0 & 0 \\
\hline $\bar{\Phi}_{12}$ & & $(1,1,1,1)$ & 8 & 0 & 0 & 0 & 0 & 0 & $(1,1)$ & 0 & 0 \\
\hline$\Phi_{23}$ & & $(1,1,1,1)$ & 4 & -12 & 0 & 0 & 0 & 0 & $(1,1)$ & 0 & 0 \\
\hline $\bar{\Phi}_{23}$ & & $(1,1,1,1)$ & -4 & 12 & 0 & 0 & 0 & 0 & $(1,1)$ & 0 & 0 \\
\hline$\Phi_{31}$ & & $(1,1,1,1)$ & -4 & -12 & 0 & 0 & 0 & 0 & $(1,1)$ & 0 & 0 \\
\hline $\bar{\Phi}_{31}$ & & $(1,1,1,1)$ & 4 & 12 & 0 & 0 & 0 & 0 & $(1,1)$ & 0 & 0 \\
\hline$H_{1}$ & $S+\beta \pm \gamma$ & $(1,1,1,1)$ & 0 & 0 & -10 & -2 & 2 & -2 & $(4,1)$ & 0 & -12 \\
\hline $\bar{H}_{1}$ & $\oplus(\zeta)$ & $(1,1,1,1)$ & 0 & 0 & 10 & 2 & -2 & 2 & $(\overline{4}, 1)$ & 0 & 12 \\
\hline$H_{2}$ & & $(1,1,1,1)$ & 0 & 0 & 2 & -2 & 2 & 2 & $(1,4)$ & 0 & 36 \\
\hline$H_{2}$ & & $(1,1,1,1)$ & 0 & 0 & -2 & 2 & -2 & -2 & $(1, \overline{4})$ & 0 & -36 \\
\hline
\end{tabular}

Table 2 continued: Model 2 fields. 


\section{Left-Right Symmetric Model 2 Superpotential Terms}

$W_{3}$ (observable):

$$
\begin{array}{llllll}
h_{1} h_{2} \Phi_{3} & +h_{1} Q_{L_{1}} Q_{R_{1}} & +h_{1} L_{L_{1}} L_{R_{1}} & +h_{1} S_{L_{1}} S_{R_{1}} & +h_{1} \mathcal{L}_{L_{1}} \overline{\mathcal{L}}_{R_{1}} & +h_{1} \overline{\mathcal{L}}_{L_{1}} \mathcal{L}_{R_{1}} \\
+h_{2} Q_{L_{2}} Q_{R_{2}} & +h_{2} L_{L_{2}} L_{R_{2}} & +h_{2} S_{L_{2}} S_{R_{2}} & +h_{2} \mathcal{L}_{L_{2}} \overline{\mathcal{L}}_{R_{2}} & +h_{2} \overline{\mathcal{L}}_{L_{2}} \mathcal{L}_{R_{2}} & +Q_{L_{1}} \bar{D}_{1} S_{L_{1}} \\
+Q_{L_{2}} \bar{D}_{2} S_{L_{2}} & +Q_{L_{3}} \bar{D}_{3} S_{L_{3}} & +Q_{R_{1}} D_{1} S_{R_{1}} & +Q_{R_{2}} D_{2} S_{R_{2}} & +Q_{R_{3}} D_{3} S_{R_{3}} & +D_{\alpha \beta} \bar{D}_{\alpha \beta} \Phi_{3} \\
+D_{\alpha \beta} \bar{D}_{3} \phi_{4} & +D_{1} \bar{D}_{2} \bar{\Phi}_{12} & +D_{1} \bar{D}_{3} \bar{\Phi}_{31} & +D_{2} \bar{D}_{1} \Phi_{12} & +D_{2} \bar{D}_{3} \bar{\Phi}_{23} & +D_{3} \bar{D}_{\alpha \beta} \bar{\phi}_{4} \\
+D_{3} \bar{D}_{1} \Phi_{31} & +D_{3} \bar{D}_{2} \Phi_{23} & +\mathcal{L}_{L_{1}} \overline{\mathcal{L}}_{L_{1}} \Phi_{1} & +\overline{\mathcal{L}}_{L_{1}} \overline{\mathcal{L}}_{L_{2}} \bar{\phi}_{4} & +\mathcal{L}_{L_{2}} \overline{\mathcal{L}}_{L_{2}} \Phi_{2} & +\mathcal{L}_{R_{1}} \overline{\mathcal{L}}_{R_{1}} \Phi_{1} \\
+\mathcal{L}_{R_{1}} \mathcal{L}_{R_{2}} \phi_{4} & +\mathcal{L}_{R_{2}} \overline{\mathcal{L}}_{R_{2}} \Phi_{2} & +N_{\alpha \beta} \bar{N}_{\alpha \beta} \Phi_{3} & & &
\end{array}
$$

$W_{3}$ (singlets):

$$
\begin{array}{rlllll}
\Phi_{3} \phi_{2}^{+} \bar{\phi}_{2}^{+} & +\Phi_{3} \phi_{2}^{-} \bar{\phi}_{2}^{-} & +\Phi_{3} \phi_{1}^{+} \bar{\phi}_{1}^{+} & +\Phi_{3} \phi_{1}^{-} \bar{\phi}_{1}^{-} & +\Phi_{3} \phi_{3}^{+} \bar{\phi}_{3}^{+} & +\Phi_{3} \phi_{3}^{-} \bar{\phi}_{3}^{-} \\
+\Phi_{3} \phi_{4} \bar{\phi}_{4} & +\Phi_{31} \bar{\Phi}_{23} \bar{\Phi}_{12} & +\bar{\Phi}_{31} \Phi_{23} \Phi_{12} & +\Phi_{12} \phi_{2}^{+} \phi_{2}^{-} & +\Phi_{12} \phi_{1}^{+} \phi_{1}^{-} & +\Phi_{12} \phi_{3}^{+} \phi_{3}^{-}(\mathrm{D} .2) \\
+\bar{\Phi}_{12} \bar{\phi}_{2}^{+} \bar{\phi}_{2}^{-} & +\bar{\Phi}_{12} \bar{\phi}_{1}^{+} \bar{\phi}_{1}^{-} & +\bar{\Phi}_{12} \bar{\phi}_{3}^{+} \bar{\phi}_{3}^{-} & & &
\end{array}
$$

$W_{3}($ hidden $)$ : none

$W_{4}($ observable):

$$
\begin{aligned}
& Q_{L_{1}} Q_{L_{1}} D_{\alpha \beta} \phi_{1}^{+} \quad+Q_{L_{1}} Q_{L_{3}} Q_{R_{1}} Q_{R_{3}} \quad+Q_{L_{1}} Q_{R_{1}} L_{L_{3}} L_{R_{3}} \quad+Q_{L_{1}} Q_{R_{1}} S_{L_{3}} S_{R_{3}} \quad+Q_{L_{2}} Q_{L_{2}} D_{\alpha \beta} \bar{\phi}_{2}^{-} \\
& +Q_{L_{2}} Q_{L_{3}} Q_{R_{2}} Q_{R_{3}}+Q_{L_{2}} Q_{R_{2}} L_{L_{3}} L_{R_{3}} \quad+Q_{L_{2}} Q_{R_{2}} S_{L_{3}} S_{R_{3}} \quad+Q_{L_{3}} Q_{R_{3}} L_{L_{1}} L_{R_{1}} \quad+Q_{L_{3}} Q_{R_{3}} L_{L_{2}} L_{R_{2}} \\
& +Q_{L_{3}} Q_{R_{3}} S_{L_{1}} S_{R_{1}} \quad+Q_{L_{3}} Q_{R_{3}} S_{L_{2}} S_{R_{2}} \quad+Q_{L_{3}} Q_{R_{3}} \mathcal{L}_{L_{1}} \overline{\mathcal{L}}_{R_{1}} \quad+Q_{L_{3}} Q_{R_{3}} \overline{\mathcal{L}}_{L_{1}} \mathcal{L}_{R_{1}} \quad+Q_{L_{3}} Q_{R_{3}} \mathcal{L}_{L_{2}} \overline{\mathcal{L}}_{R_{2}} \\
& +Q_{L_{3}} Q_{R_{3}} \overline{\mathcal{L}}_{L_{2}} \mathcal{L}_{R_{2}} \quad+Q_{R_{1}} Q_{R_{1}} \bar{D}_{\alpha \beta} \bar{\phi}_{1}^{+} \quad+Q_{R_{2}} Q_{R_{2}} \bar{D}_{\alpha \beta} \phi_{2}^{-} \quad+L_{L_{1}} L_{L_{1}} \phi_{1}^{-} \phi_{4} \quad+L_{L_{1}} L_{L_{3}} L_{R_{1}} L_{R_{3}} \\
& +L_{L_{1}} L_{R_{1}} S_{L_{3}} S_{R_{3}}+L_{L_{2}} L_{L_{2}} \bar{\phi}_{2}^{+} \phi_{4} \quad+L_{L_{2}} L_{L_{3}} L_{R_{2}} L_{R_{3}} \quad+L_{L_{2}} L_{R_{2}} S_{L_{3}} S_{R_{3}} \quad+L_{L_{3}} L_{R_{3}} S_{L_{1}} S_{R_{1}} \\
& +L_{L_{3}} L_{R_{3}} S_{L_{2}} S_{R_{2}} \quad+L_{L_{3}} L_{R_{3}} \mathcal{L}_{L_{1}} \overline{\mathcal{L}}_{R_{1}} \quad+L_{L_{3}} L_{R_{3}} \overline{\mathcal{L}}_{L_{1}} \mathcal{L}_{R_{1}} \quad+L_{L_{3}} L_{R_{3}} \mathcal{L}_{L_{2}} \overline{\mathcal{L}}_{R_{2}} \quad+L_{L_{3}} L_{R_{3}} \overline{\mathcal{L}}_{L_{2}} \mathcal{L}_{R_{2}} \\
& +L_{R_{1}} L_{R_{1}} \bar{\phi}_{1}^{-} \bar{\phi}_{4}+L_{R_{2}} L_{R_{2}} \phi_{2}^{+} \bar{\phi}_{4} \quad+S_{L_{1}} S_{L_{3}} S_{R_{1}} S_{R_{3}} \quad+S_{L_{2}} S_{L_{3}} S_{R_{2}} S_{R_{3}} \quad+S_{L_{3}} \mathcal{L}_{L_{1}} S_{R_{3}} \overline{\mathcal{L}}_{R_{1}} \\
& +S_{L_{3}} \overline{\mathcal{L}}_{L_{1}} S_{R_{3}} \mathcal{L}_{R_{1}} \quad+S_{L_{3}} \mathcal{L}_{L_{2}} S_{R_{3}} \overline{\mathcal{L}}_{R_{2}} \quad+S_{L_{3}} \overline{\mathcal{L}}_{L_{2}} S_{R_{3}} \mathcal{L}_{R_{2}}
\end{aligned}
$$

$W_{4}$ (singlets), $W_{4}$ (mixed), $W_{4}($ hidden $)$ : none 
$W_{5}$ (observable):

\begin{tabular}{|c|c|c|c|}
\hline$h_{1} Q_{L_{2}} Q_{R_{2}} D_{\alpha \beta} \bar{D}_{\alpha \beta}$ & $+h_{1} Q_{L_{2}} Q_{R_{2}} N_{\alpha \beta} N_{\alpha \beta}$ & $+h_{1} Q_{L_{2}} Q_{R_{2}} \phi_{2}^{+} \phi_{2}^{+}$ & $+h_{1} Q_{L_{2}} Q_{R_{2}} \phi_{2}^{-} \phi_{2}^{-}$ \\
\hline$h_{1} Q_{L_{2}} Q_{R_{2}} \phi_{1}^{+} \bar{\phi}_{1}^{+}$ & $+h_{1} Q_{L_{2}} Q_{R_{2}} \phi_{1}^{-} \bar{\phi}_{1}^{-}$ & $+h_{1} Q_{L_{2}} Q_{R_{2}} \phi_{3}^{+} \bar{\phi}_{3}^{+}$ & $+h_{1} Q_{L_{2}} Q_{R_{2}} \phi_{3}^{-} \bar{\phi}_{3}^{-}$ \\
\hline$h_{1} Q_{L_{2}} Q_{R_{2}} \phi_{4} \bar{\phi}_{4}$ & $+h_{1} L_{L_{2}} L_{R_{2}} D_{\alpha \underline{\beta}} \bar{D}_{\alpha}$ & $+h_{1} L_{L_{2}} L_{R_{2}} N_{\alpha \underline{\beta}} \bar{N}_{c}$ & $+h_{1} L_{L_{2}} L_{R_{2}} \phi_{2}^{+} \bar{\phi}_{2}^{+}$ \\
\hline$h_{1} L_{L_{2}} L_{R_{2}} \phi_{2}^{-} \bar{\phi}_{2}^{-}$ & $+h_{1} L_{L_{2}} L_{R_{2}} \phi_{1}^{+} \bar{\phi}_{1}^{+}$ & $+h_{1} L_{L_{2}} L_{R_{2}} \phi_{1}^{-} \bar{\phi}_{1}^{-}$ & $+h_{1} L_{L_{2}} L_{R_{2}} \phi_{3}^{+} \bar{\phi}_{3}^{+}$ \\
\hline$h_{1} L_{L_{2}} L_{R_{2}} \phi_{\underline{3}}^{-} \bar{\phi}_{3}^{-}$ & $+h_{1} L_{L_{2}} L_{R_{2}} \phi_{4} \bar{\phi}_{4}$ & $+h_{1} D_{\alpha \beta} \bar{D}_{\alpha \beta} S_{L_{2}} S_{R_{2}}$ & $+h_{1} D_{\alpha \beta} \bar{D}_{\alpha \beta} \mathcal{L}_{L_{2}} \overline{\mathcal{L}}_{R_{2}}$ \\
\hline$h_{1} D_{\alpha \beta} \bar{D}_{\alpha \beta} \overline{\mathcal{L}}_{L_{2}} \mathcal{L}_{R_{2}}$ & $+h_{1} S_{L_{2}} S_{R_{2}} N_{\alpha \beta} \bar{N}_{\alpha \beta}$ & $S_{L_{2}} S_{R_{2}} \phi_{2}^{+} \bar{\phi}_{2}^{+}$ & $S_{L_{2}} S_{R_{2}} \phi_{2}^{-} \bar{\phi}_{2}^{-}$ \\
\hline$h_{1} S_{L_{2}} S_{R_{2}} \phi_{1}^{+} \bar{\phi}_{1}^{+}$ & $+h_{1} S_{L_{2}} S_{R_{2}} \phi_{1}^{-} \bar{\phi}_{1}^{-}$ & $+h_{1} S_{L_{2}} S_{R_{2}} \phi_{3}^{+} \bar{\phi}_{3}^{+}$ & $+h_{1} S_{L_{2}} S_{R_{2}} \phi_{3}^{-} \bar{\phi}_{3}^{-}$ \\
\hline$h_{1} S_{L_{2}} S_{R_{2}} \phi_{4} \bar{\phi}_{4}$ & $+h_{1} \mathcal{L}_{L_{2}} \overline{\mathcal{L}}_{R_{2}} N_{\alpha \beta} \bar{N}_{\alpha \beta}$ & $+h_{1} \mathcal{L}_{L_{2}} \overline{\mathcal{L}}_{R_{2}} \phi_{2}^{+} \bar{\phi}_{2}^{+}$ & $+h_{1} \mathcal{L}_{L_{2}} \overline{\mathcal{L}}_{R_{2}} \phi_{2}^{-} \bar{\phi}_{2}^{-}$ \\
\hline$h_{1} \mathcal{L}_{L_{2}} \overline{\mathcal{L}}_{R_{2}} \phi_{1}^{+} \bar{\phi}_{1}^{+}$ & $+h_{1} \mathcal{L}_{L_{2}} \overline{\mathcal{L}}_{R_{2}} \phi_{1}^{-} \bar{\phi}_{1}^{-}$ & $+h_{1} \mathcal{L}_{L_{2}} \overline{\mathcal{L}}_{R_{2}} \phi_{3}^{+} \bar{\phi}_{3}^{+}$ & $+h_{1} \mathcal{L}_{L_{2}} \overline{\mathcal{L}}_{l}$ \\
\hline$h_{1} \mathcal{L}_{L_{2}} \overline{\mathcal{L}}_{R_{2}} \phi_{4} \bar{\phi}_{4}$ & $+h_{1} \overline{\mathcal{L}}_{L_{2}} \mathcal{L}_{R_{2}} N_{\alpha \beta} \bar{N}_{\alpha \beta}$ & $+h_{1} \overline{\mathcal{L}}_{L_{2}} \mathcal{L}_{R_{2}} \phi_{2}^{+} \bar{\phi}_{2}^{+}$ & $+h_{1} \overline{\mathcal{L}}_{L_{2}} \mathcal{L}_{R_{2}} \phi_{2}^{-} \bar{\phi}_{2}^{-}$ \\
\hline$h_{1} \overline{\mathcal{L}}_{L_{2}} \mathcal{L}_{R_{2}} \phi_{1}^{+} \bar{\phi}_{1}^{+}$ & $+h_{1} \overline{\mathcal{L}}_{L_{2}} \mathcal{L}_{R_{2}} \phi_{1}^{-} \bar{\phi}_{1}^{-}$ & ${ }_{1} \overline{\mathcal{L}}_{L_{2}} \mathcal{L}_{R_{2}} \phi_{3}^{+} \bar{\phi}_{3}^{+}$ & ${ }_{1} \overline{\mathcal{L}}_{L_{2}} \mathcal{L}_{I}$ \\
\hline$h_{1} \overline{\mathcal{L}}_{L_{2}} \mathcal{L}_{R_{2}} \phi_{4} \bar{\phi}_{\underline{4}}$ & $+h_{2} Q_{L_{1}} Q_{R_{1}} D_{\alpha \underline{\beta}} \bar{D}_{\alpha \beta}$ & $+h_{2} Q_{L_{1}} Q_{R_{1}} N_{\alpha \underline{\beta}} \bar{N}_{\alpha \beta}$ & $+h_{2} Q_{L_{1}} Q_{R_{1}} \phi_{2}^{+} \bar{\phi}_{2}^{+}$ \\
\hline$h_{2} Q_{L_{1}} Q_{R_{1}} \phi_{2}^{-} \bar{\phi}_{2}^{-}$ & $+h_{2} Q_{L_{1}} Q_{R_{1}} \phi_{1}^{+} \bar{\phi}_{1}^{+}$ & $h_{2} Q_{L_{1}} Q_{R_{1}} \phi_{1}^{-} \bar{\phi}_{\underline{1}}^{-}$ & $h_{2} Q_{L_{1}} Q_{R_{1}} \phi_{3}^{+} \bar{\phi}_{\underline{3}}^{+}$ \\
\hline$h_{2} Q_{L_{1}} Q_{R_{1}} \phi_{3}^{-} \bar{\phi}_{3}^{-}$ & $+h_{2} Q_{L_{1}} Q_{R_{1}} \phi_{4} \bar{\phi}_{4}$ & ${ }_{2} L_{L_{1}} L_{R_{1}} D_{\alpha \beta} \overline{\bar{D}}_{\alpha}$ & $+h_{2} L_{L_{1}} L_{R_{1}} N_{\alpha \beta} \bar{N}_{\alpha}$ \\
\hline$h_{2} L_{L_{1}} L_{R_{1}} \phi_{2}^{+} \bar{\phi}_{2}^{+}$ & $+h_{2} L_{L_{1}} L_{R_{1}} \phi_{2}^{-} \bar{\phi}_{2}^{-}$ & $+h_{2} L_{L_{1}} L_{R_{1}} \phi_{1}^{+} \bar{\phi}_{1}^{+}$ & $+h_{2} L_{L_{1}} L_{R_{1}} \phi_{1}^{-\bar{\phi}}$ \\
\hline${ }_{2} L_{L_{1}} L_{R_{1}} \phi_{3}^{+} \bar{\phi}_{3}^{+}$ & $+h_{2} L_{L_{1}} L_{R_{1}} \phi_{3}^{-} \bar{\phi}_{3}^{-}$ & $L_{L_{1}} L_{R_{1}} \phi_{4} \bar{\phi}_{4}$ & $+h_{2} D_{\alpha \beta} \bar{D}_{\alpha \beta} S_{L_{1}} S_{R_{1}}$ \\
\hline$h_{2} D_{\alpha \beta} \bar{D}_{\alpha \beta} \mathcal{L}_{L_{1}} \overline{\mathcal{L}}_{R_{1}}$ & $+h_{2} D_{\alpha \beta} \bar{D}_{\alpha \beta} \overline{\mathcal{L}}_{L_{1}} \mathcal{L}_{R_{1}}$ & $S_{L_{1}} S_{R_{1}} N_{\alpha \beta} \bar{N}_{\alpha \beta}$ & ${ }_{2} S_{L_{1}} S_{R_{1}} \phi_{2}^{+}$ \\
\hline$\imath_{2} S_{L_{1}} S_{R_{1}} \phi_{2}^{-} \bar{\phi}_{2}^{-}$ & $+h_{2} S_{L_{1}} S_{R_{1}} \phi_{1}^{+} \bar{\phi}_{1}^{+}$ & $S_{L_{1}} S_{R}$ & $+h_{2} S_{L_{1}} S_{R}$ \\
\hline$L_{1} S_{R_{1}}$ & $+h_{2} S_{L_{1}} S_{R_{1}} \phi_{4} \bar{\phi}_{4}$ & $+h_{2} \mathcal{L}_{L_{1}} \overline{\mathcal{L}}_{R_{1}} N_{\alpha \beta} \bar{N}_{\alpha \beta}$ & $+h_{2} \mathcal{L}_{L_{1}} \overline{\mathcal{L}}_{R_{1}} \phi_{2}^{+} \bar{\phi}_{2}^{+}$ \\
\hline$h_{2} \mathcal{L}_{L_{1}} \overline{\mathcal{L}}_{R_{1}} \phi_{2}^{-} \bar{\phi}_{2}^{-}$ & $+h_{2} \mathcal{L}_{L_{1}} \overline{\mathcal{L}}_{R_{1}}$ & $+h_{2} \mathcal{L}_{L_{1}} \overline{\mathcal{L}}_{H}$ & $i_{2} \mathcal{L}_{L_{1}} \overline{\mathcal{L}}$ \\
\hline$h_{2} \mathcal{L}_{L_{1}} \overline{\mathcal{L}}_{R_{1}} \phi$ & $+h_{2} \mathcal{L}_{L_{1}} \overline{\mathcal{L}}_{R}$ & $+h_{2} \overline{\mathcal{L}}_{L_{1}} \mathcal{L}_{R_{1}} N_{\alpha \beta} \bar{N}_{\alpha \beta}$ & $+h_{2} \overline{\mathcal{L}}_{L_{1}} \mathcal{L}_{R_{1}} \phi_{2}^{+} \bar{\phi}_{2}^{+}$ \\
\hline$h_{2} \overline{\mathcal{L}}_{L_{1}} \mathcal{L}_{R_{1}}$ & $+h_{2} \overline{\mathcal{L}}_{L_{1}} \mathcal{L}_{R}$ & $+h_{2} \overline{\mathcal{L}}_{L_{1}} \mathcal{L}_{I}$ & $+h_{2} \overline{\mathcal{L}}_{L_{1}} \mathcal{L}_{R_{1}} \phi_{3}^{+} \bar{\phi}_{3}^{+}$ \\
\hline$h_{2} \overline{\mathcal{L}}_{L_{1}} \mathcal{L}_{R_{1}} \phi$ & $+h_{2} \overline{\mathcal{L}}_{L_{1}} \mathcal{L}_{R}$ & $Q_{L_{1}} Q_{L_{2}} Q_{R_{1}} Q_{R_{2}} \Phi_{3}$ & $+Q_{L_{1}} Q_{L_{2}} L_{L_{1}} L_{L_{2}} D_{3}$ \\
\hline$Q_{L_{1}} Q_{R_{1}} L_{L_{2}} L_{R_{2}} \Phi_{3}$ & $+Q_{L_{1}} Q_{R_{1}} S_{L_{2}} S_{R_{2}} \Phi_{3}$ & $+Q_{L_{1}} Q_{R_{1}} \mathcal{L}_{L_{1}} \mathcal{L}_{R_{2}} \phi_{4}$ & $+Q_{L_{1}} Q_{R_{1}} \mathcal{L}_{L_{2}} \overline{\mathcal{L}}_{R_{2}} \Phi_{3}$ \\
\hline$Q_{L_{1}} Q_{R_{1}} \overline{\mathcal{L}}_{L_{2}} \overline{\mathcal{L}}_{R_{1}} \bar{\phi}_{4}$ & $+Q_{L_{1}} Q_{R_{1}} \overline{\mathcal{L}}_{L_{2}} \mathcal{L}_{R_{2}} \Phi_{3}$ & $+Q_{L_{1}} Q_{R_{2}} L_{L_{1}} L_{R_{2}} \bar{\Phi}_{12}$ & $+Q_{L_{1}} Q_{R_{2}} S_{L}$ \\
\hline$Q_{L_{1}} Q_{R_{3}} S_{L_{1}} S_{R_{3}} \bar{\Phi}_{31}$ & $+Q_{L_{1}} \bar{D}_{2} S_{L_{1}} \phi_{2}^{+} \phi_{2}^{-}$ & $Q_{L_{1}} \bar{D}_{2} S_{L_{1}} \phi_{1}^{+} \phi_{1}^{-}$ & $+Q_{L_{1}} \bar{D}_{2} S_{L_{1}} \phi_{3}^{+} \phi_{3}^{-}$ \\
\hline$Q_{L_{2}} Q_{R_{1}} L_{L_{2}} L_{R_{1}} \Phi_{12}$ & $+Q_{L_{2}} Q_{R_{1}} S_{L_{2}} S_{R_{1}} \Phi_{12}$ & $+Q_{L_{2}} Q_{R_{2}} L_{L_{1}} L_{R_{1}} \Phi_{3}$ & $+Q_{L_{2}} Q_{R_{2}} S_{L_{1}}$ \\
\hline$Q_{L_{2}} Q_{R_{2}} \mathcal{L}_{L_{1}} \overline{\mathcal{L}}_{R_{1}} \Phi_{3}$ & $+Q_{L_{2}} Q_{R_{2}} \overline{\mathcal{L}}_{L_{1}} \mathcal{L}_{R_{1}}$ & & \\
\hline$Q_{L_{2}} Q_{R_{3}} S_{L_{2}} S_{R_{3}} \bar{\Phi}_{23}$ & $+Q_{L_{2}} \bar{D}_{\alpha \beta} S_{L_{2}} \bar{\Phi}_{23} \bar{\phi}_{4}$ & $-Q_{L_{2}} \bar{D}_{1} S$ & $+Q_{L_{2}} \bar{D}_{1} S_{L_{2}} \bar{\phi}_{1}^{+} \bar{\phi}_{1}^{-}$ \\
\hline$-Q_{L_{2}} \bar{D}_{1} S_{L_{2}} \bar{\phi}_{3}^{+} \bar{\phi}_{3}^{-}$ & $+Q_{L_{3}} Q_{R_{1}} S_{L_{3}} S_{R_{1}}$ & $+Q_{L_{3}} Q_{R_{2}}$ & $+Q_{L_{3}} \bar{D}$ \\
\hline$Q_{R_{1}} Q_{R_{2}} L_{R_{1}} L_{R_{2}} \bar{D}_{3}$ & $+Q_{R_{1}} D_{\alpha \beta} S$ & $\bar{\phi}_{2}^{+} \bar{\phi}_{2}^{-}$ & $R_{1} \bar{\phi}_{1}^{+} \bar{\phi}_{1}^{-}$ \\
\hline$Q_{R_{1}} D_{2} S_{R_{1}} \bar{\phi}_{3}^{+} \bar{\phi}_{3}^{-}$ & $+Q_{R_{2}} D_{\alpha \beta} S_{R_{2}} \Phi_{23} \phi_{4}$ & $+Q_{R_{2}} L$ & $+Q_{R_{2}} D_{1} S_{R_{2}} \phi_{1}^{+} \phi_{1}^{-}$ \\
\hline$Q_{R_{2}} D_{1} S_{R_{2}} \phi_{3}^{+} \phi_{3}^{-}$ & $+Q_{R_{3}} D_{\alpha \beta} \overline{\mathcal{L}}_{L_{1}} \overline{\mathcal{L}}_{L_{2}}$ & $+L_{L_{1}} L_{L_{2}}$ & $+L_{L_{1}} L_{R_{1}} S_{L_{2}} S_{R_{2}} \Phi_{3}$ \\
\hline$r_{2} \phi_{4}$ & $+L_{L}$ & $+L_{L}$ & $+L_{L_{1}}$ \\
\hline$S_{L_{2}} S_{R_{1}} \Phi_{3}$ & $+L_{L_{1}} L$ & $+L_{L_{1}} S$ & $+L_{I}$ \\
\hline$L_{L_{2}} L_{R_{2}} S_{L_{1}} S_{R_{1}} \Phi_{3}$ & $+L_{L_{2}} L_{R_{2}} \mathcal{L}_{L_{1}} \overline{\mathcal{L}}_{R_{1}} \Phi_{3}$ & $+L_{L_{2}} L_{R_{2}} L$ & $+L_{L_{2}} L$ \\
\hline & $+L_{L_{2}} L$ & $I=S$ & +1 \\
\hline$S_{L_{2}} S_{R_{3}} \Phi_{1}$ & $\Phi_{2} \bar{\phi}_{1}^{-}$ & $+L_{R_{0}} S$ & $+D_{\alpha}$ \\
\hline$\overline{\mathcal{L}}_{R_{1}} \Phi$ & $+D_{\alpha \beta} \bar{D}$ & $+S_{L_{1}}$ & $+S_{L}$ \\
\hline$\overline{\mathcal{L}}_{R_{2}} \Phi$ & + & $+S_{L_{1}}$ & $+S$ \\
\hline$L_{2}$ & $+S_{L_{2}} \overline{\mathcal{L}}$ & $S_{L}$ & $+\mathcal{L}$ \\
\hline$c$ & $\mathcal{C}$ & & $+\mathcal{L}$ \\
\hline
\end{tabular}


$W_{5}$ (observable) continued:

$$
\begin{array}{llll}
+\mathcal{L}_{L_{1}} \overline{\mathcal{L}}_{L_{2}} \overline{\mathcal{L}}_{R_{1}} \mathcal{L}_{R_{2}} \Phi_{3} & +\overline{\mathcal{L}}_{L_{1}} \mathcal{L}_{L_{2}} \overline{\mathcal{L}}_{L_{2}} \overline{\mathcal{L}}_{L_{2}} \bar{\phi}_{4} & +\overline{\mathcal{L}}_{L_{1}} \mathcal{L}_{L_{2}} \mathcal{L}_{R_{1}} \overline{\mathcal{L}}_{R_{2}} \Phi_{3} & +\overline{\mathcal{L}}_{L_{1}} \mathcal{L}_{L_{2}} \overline{\mathcal{L}}_{R_{2}} \overline{\mathcal{L}}_{R_{2}} \bar{\phi}_{4} \\
+\overline{\mathcal{L}}_{L_{1}} \overline{\mathcal{L}}_{L_{2}} \mathcal{L}_{R_{1}} \overline{\mathcal{L}}_{R_{1}} \bar{\phi}_{4} & +\overline{\mathcal{L}}_{L_{1}} \overline{\mathcal{L}}_{L_{2}} \mathcal{L}_{R_{1}} \mathcal{L}_{R_{2}} \Phi_{3} & +\overline{\mathcal{L}}_{L_{1}} \overline{\mathcal{L}}_{L_{2}} \mathcal{L}_{R_{2}} \overline{\mathcal{L}}_{R_{2}} \bar{\phi}_{4} & +\overline{\mathcal{L}}_{L_{1}} \overline{\mathcal{L}}_{L_{2}} N_{\alpha \beta} \bar{N}_{\alpha \beta} \bar{\phi}_{4} \\
+\overline{\mathcal{L}}_{L_{1}} \overline{\mathcal{L}}_{L_{2}} \phi_{2}^{+} \bar{\phi}_{2}^{+} \bar{\phi}_{4} & +\overline{\mathcal{L}}_{L_{1}} \overline{\mathcal{L}}_{L_{2}} \phi_{2}^{-} \bar{\phi}_{2}^{-} \bar{\phi}_{4} & +\overline{\mathcal{L}}_{L_{1}} \overline{\mathcal{L}}_{L_{2}} \phi_{1}^{+} \bar{\phi}_{1}^{+} \bar{\phi}_{4} & +\overline{\mathcal{L}}_{L_{1}} \overline{\mathcal{L}}_{L_{2}} \phi_{1}^{-} \bar{\phi}_{1}^{-} \bar{\phi}_{4} \\
+\overline{\mathcal{L}}_{L_{1}} \overline{\mathcal{L}}_{L_{2}} \phi_{3}^{+} \bar{\phi}_{3}^{+} \bar{\phi}_{4} & +\overline{\mathcal{L}}_{L_{1}} \overline{\mathcal{L}}_{L_{2}} \phi_{3}^{-} \bar{\phi}_{3}^{-} \bar{\phi}_{4} & +\overline{\mathcal{L}}_{L_{1}} \overline{\mathcal{L}}_{L_{2}} \phi_{4} \bar{\phi}_{4} \bar{\phi}_{4} & +\mathcal{L}_{L_{2}} \mathcal{L}_{L_{2}} \mathcal{L}_{R_{1}} \overline{\mathcal{L}}_{R_{2}} \phi_{4}(\mathrm{D} .4) \\
+\mathcal{L}_{L_{2}} \overline{\mathcal{L}}_{L_{2}} \mathcal{L}_{R_{1}} \mathcal{L}_{R_{2}} \phi_{4} & +\mathcal{L}_{R_{1}} \mathcal{L}_{R_{1}} \overline{\mathcal{L}}_{R_{1}} \mathcal{L}_{R_{2}} \phi_{4} & +\mathcal{L}_{R_{1}} \mathcal{L}_{R_{2}} \mathcal{L}_{R_{2}} \overline{\mathcal{L}}_{R_{2}} \phi_{4} & +\mathcal{L}_{R_{1}} \mathcal{L}_{R_{2}} N_{\alpha \beta} \bar{N}_{\alpha \beta} \phi_{4} \\
+\mathcal{L}_{R_{1}} \mathcal{L}_{R_{2}} \phi_{2}^{+} \bar{\phi}_{2}^{+} \phi_{4} & +\mathcal{L}_{R_{1}} \mathcal{L}_{R_{2}} \phi_{2}^{-} \bar{\phi}_{2}^{-} \phi_{4} & +\mathcal{L}_{R_{1}} \mathcal{L}_{R_{2}} \phi_{1}^{+} \bar{\phi}_{1}^{+} \phi_{4} & +\mathcal{L}_{R_{1}} \mathcal{L}_{R_{2}} \phi_{1}^{-} \bar{\phi}_{1}^{-} \phi_{4} \\
+\mathcal{L}_{R_{1}} \mathcal{L}_{R_{2}} \phi_{3}^{+} \bar{\phi}_{3}^{+} \phi_{4} & +\mathcal{L}_{R_{1}} \mathcal{L}_{R_{2}} \phi_{3}^{-} \bar{\phi}_{3}^{-} \phi_{4} & +\mathcal{L}_{R_{1}} \mathcal{L}_{R_{2}} \phi_{4} \phi_{4} \bar{\phi}_{4} &
\end{array}
$$

$W_{5}($ singlets $), W_{5}($ mixed $), W_{5}($ hidden $)$ : none 
E Left-Right Symmetric Model 3 Fields

\begin{tabular}{|c|c|c|c|c|c|c|c|c|c|c|c|c|c|c|}
\hline$F$ & SEC & $(C ; L ; R)$ & $Q_{C}$ & $Q_{1}$ & $Q_{2}$ & $Q_{3}$ & $Q_{4}$ & $Q_{5}$ & $Q_{6}$ & $S U(3)_{H_{1 ; 2}}$ & $Q_{7}$ & $Q_{8}$ & $Q_{9}$ & $Q_{10}$ \\
\hline$Q_{L_{1}}$ & $\mathbf{b}_{1}$ & $(3,2,1)$ & 2 & 2 & 0 & 0 & -2 & 0 & 0 & $(1,1)$ & 0 & 0 & 0 & 0 \\
\hline$Q_{R_{1}}$ & & $(\overline{3}, 1,2)$ & -2 & -2 & 0 & 0 & -2 & 0 & 0 & $(1,1)$ & 0 & 0 & 0 & 0 \\
\hline$L_{L_{1}}$ & & $(1,2,1)$ & -6 & 2 & 0 & 0 & -2 & 0 & 0 & $(1,1)$ & 0 & 0 & 0 & 0 \\
\hline$L_{R_{1}}$ & & $(1,1,2)$ & 6 & -2 & 0 & 0 & -2 & 0 & 0 & $(1,1)$ & 0 & 0 & 0 & 0 \\
\hline$Q_{L_{2}}$ & $\mathbf{b}_{2}$ & $(3,2,1)$ & 2 & 0 & 2 & 0 & 0 & -2 & 0 & $(1,1)$ & 0 & 0 & 0 & 0 \\
\hline$Q_{R_{2}}$ & & $(\overline{3}, 1,2)$ & -2 & 0 & -2 & 0 & 0 & -2 & 0 & $(1,1)$ & 0 & 0 & 0 & 0 \\
\hline$L_{L_{2}}$ & & $(1,2,1)$ & -6 & 0 & 2 & 0 & 0 & -2 & 0 & $(1,1)$ & 0 & 0 & 0 & 0 \\
\hline$L_{R_{2}}$ & & $(1,1,2)$ & 6 & 0 & -2 & 0 & 0 & -2 & 0 & $(1,1)$ & 0 & 0 & 0 & 0 \\
\hline$Q_{L_{3}}$ & $\mathbf{b}_{3}$ & $(3,2,1)$ & 2 & 0 & 0 & 2 & 0 & 0 & -2 & $(1,1)$ & 0 & 0 & 0 & 0 \\
\hline$Q_{R_{3}}$ & & $(\overline{3}, 1,2)$ & -2 & 0 & 0 & -2 & 0 & 0 & -2 & $(1,1)$ & 0 & 0 & 0 & 0 \\
\hline$L_{L_{3}}$ & & $(1,2,1)$ & -6 & 0 & 0 & 2 & 0 & 0 & -2 & $(1,1)$ & 0 & 0 & 0 & 0 \\
\hline$L_{R_{3}}$ & & $(1,1,2)$ & 6 & 0 & 0 & -2 & 0 & 0 & -2 & $(1,1)$ & 0 & 0 & 0 & 0 \\
\hline$h_{1}$ & Neveu- & $(1,2,2)$ & 0 & 0 & 0 & 0 & 0 & 0 & 0 & $(1,1)$ & 0 & 0 & 0 & 0 \\
\hline$h_{2}$ & Schwarz & $(1,2,2)$ & 0 & 0 & 0 & 0 & 0 & 0 & 0 & $(1,1)$ & 0 & 0 & 0 & 0 \\
\hline$\Phi_{1}$ & & $(1,1,1)$ & 0 & 0 & 0 & 0 & 0 & 0 & 0 & $(1,1)$ & 0 & 0 & 0 & 0 \\
\hline$\Phi_{2}$ & & $(1,1,1)$ & 0 & 0 & 0 & 0 & 0 & 0 & 0 & $(1,1)$ & 0 & 0 & 0 & 0 \\
\hline$\Phi_{3}$ & & $(1,1,1)$ & 0 & 0 & 0 & 0 & 0 & 0 & 0 & $(1,1)$ & 0 & 0 & 0 & 0 \\
\hline$\Phi_{12}$ & & $(1,1,1)$ & 0 & -4 & 4 & 0 & 0 & 0 & 0 & $(1,1)$ & 0 & 0 & 0 & 0 \\
\hline $\bar{\Phi}_{12}$ & & $(1,1,1)$ & 0 & 4 & -4 & 0 & 0 & 0 & 0 & $(1,1)$ & 0 & 0 & 0 & 0 \\
\hline$\Phi_{23}$ & & $(1,1,1)$ & 0 & 0 & -4 & 4 & 0 & 0 & 0 & $(1,1)$ & 0 & 0 & 0 & 0 \\
\hline $\bar{\Phi}_{23}$ & & $(1,1,1)$ & 0 & 0 & 4 & -4 & 0 & 0 & 0 & $(1,1)$ & 0 & 0 & 0 & 0 \\
\hline$\Phi_{31}$ & & $(1,1,1)$ & 0 & -4 & 0 & 4 & 0 & 0 & 0 & $(1,1)$ & 0 & 0 & 0 & 0 \\
\hline $\bar{\Phi}_{31}$ & & $(1,1,1)$ & 0 & 4 & 0 & -4 & 0 & 0 & 0 & $(1,1)$ & 0 & 0 & 0 & 0 \\
\hline $\mathcal{L}_{L_{12}}^{+}$ & $\mathbf{1}+\mathbf{b}_{1}$ & $(1,2,1)$ & 0 & -2 & -2 & 0 & 0 & 0 & 2 & $(1,1)$ & -2 & 0 & 0 & 2 \\
\hline $\mathcal{L}_{L_{12}}^{-12}$ & $+\mathbf{b}_{2}+2 \gamma$ & $(1,2,1)$ & 0 & -2 & -2 & 0 & 0 & 0 & -2 & $(1,1)$ & 2 & 0 & 0 & -2 \\
\hline $\mathcal{L}_{R_{12}}^{+}$ & & $(1,1,2)$ & 0 & 2 & 2 & 0 & 0 & 0 & 2 & $(1,1)$ & 2 & 0 & 0 & -2 \\
\hline $\mathcal{L}_{R_{12}}^{-}$ & & $(1,1,2)$ & 0 & 2 & 2 & 0 & 0 & 0 & -2 & $(1,1)$ & -2 & 0 & 0 & 2 \\
\hline $\mathcal{L}_{L_{13}}^{+}$ & $\mathbf{1}+\mathbf{b}_{1}$ & $(1,2,1)$ & 0 & -2 & 0 & -2 & 0 & 2 & 0 & $(1,1)$ & -2 & 0 & 0 & 2 \\
\hline $\mathcal{L}_{L_{13}}^{-}$ & $+\mathbf{b}_{3}+2 \gamma$ & $(1,2,1)$ & 0 & -2 & 0 & -2 & 0 & -2 & 0 & $(1,1)$ & 2 & 0 & 0 & -2 \\
\hline $\mathcal{L}_{R_{13}}^{+}$ & & $(1,1,2)$ & 0 & 2 & 0 & 2 & 0 & 2 & 0 & $(1,1)$ & 2 & 0 & 0 & -2 \\
\hline $\mathcal{L}_{R_{13}}^{-+}$ & & $(1,1,2)$ & 0 & 2 & 0 & 2 & 0 & -2 & 0 & $(1,1)$ & -2 & 0 & 0 & 2 \\
\hline $\mathcal{L}_{L_{23}}^{+}$ & $1+b_{2}$ & $(1,2,1)$ & 0 & 0 & -2 & -2 & 2 & $\overline{0}$ & 0 & $(1,1)$ & -2 & 0 & 0 & 2 \\
\hline $\mathcal{L}_{L_{23}}^{-}$ & $+\mathbf{b}_{3}+2 \gamma$ & $(1,2,1)$ & 0 & 0 & -2 & -2 & -2 & 0 & 0 & $(1,1)$ & 2 & 0 & 0 & -2 \\
\hline $\mathcal{L}_{R_{23}}^{+}$ & & $(1,1,2)$ & 0 & 0 & 2 & 2 & 2 & 0 & 0 & $(1,1)$ & 2 & 0 & 0 & -2 \\
\hline $\mathcal{L}_{R_{23}}^{-}$ & & $(1,1,2)$ & 0 & 0 & 2 & 2 & -2 & 0 & 0 & $(1,1)$ & -2 & 0 & 0 & 2 \\
\hline$D_{1}$ & $\mathbf{1}+\mathbf{S}+\mathbf{b}_{2}$ & $(3,1,1)$ & -3 & 1 & -1 & 1 & 0 & 0 & 0 & $(1,1)$ & 0 & -3 & 3 & 2 \\
\hline $\bar{D}_{1}$ & $+\alpha \pm \gamma$ & $(\overline{3}, 1,1)$ & 3 & -1 & 1 & -1 & 0 & 0 & 0 & $(1,1)$ & 0 & 3 & -3 & -2 \\
\hline$D_{2}$ & $\mathbf{1}+\mathbf{S}+\mathbf{b}_{3}$ & $(3,1,1)$ & 2 & 0 & 0 & -2 & 0 & 0 & 0 & $(1,1)$ & 2 & 0 & 0 & 2 \\
\hline $\bar{D}_{2}$ & $+\alpha+\beta+2 \gamma$ & $(\overline{3}, 1,1)$ & -2 & 0 & 0 & 2 & 0 & 0 & 0 & $(1,1)$ & -2 & 0 & 0 & -2 \\
\hline
\end{tabular}

Table 3: Model 3 fields. 


\begin{tabular}{|c|c|c|c|c|c|c|c|c|c|c|c|c|c|c|}
\hline$F$ & SEC & $(C ; L ; R)$ & $Q_{C}$ & $Q_{1}$ & $Q_{2}$ & $Q_{3}$ & $Q_{4}$ & $\overline{Q_{5}}$ & $\overline{Q_{6}}$ & $\overline{S U(3)_{H_{1 ; 2}}}$ & $\overline{Q_{7}}$ & $\overline{Q_{8}}$ & $Q_{9}$ & $Q_{10}$ \\
\hline$\phi_{1}$ & $\mathbf{S}+\mathbf{b}_{1}+\mathbf{b}_{2}$ & $(1,1,1)$ & 0 & 0 & 0 & -2 & -2 & -2 & 0 & $(1,1)$ & -2 & 0 & 6 & 0 \\
\hline$\phi_{2}$ & $+\alpha+2 \gamma$ & $(1,1,1)$ & 0 & 0 & 0 & 2 & -2 & -2 & 0 & $(1,1)$ & 2 & 0 & -6 & 0 \\
\hline$\phi_{3}$ & $\mathbf{S}+\mathbf{b}_{1}+\mathbf{b}_{3}$ & $(1,1,1)$ & 0 & 0 & -2 & 0 & -2 & $\overline{0}$ & -2 & $(1,1)$ & -2 & 0 & 6 & $\overline{0}$ \\
\hline$\phi_{4}$ & $+\alpha+2 \gamma$ & $(1,1,1)$ & 0 & 0 & 2 & 0 & -2 & 0 & -2 & $(1,1)$ & 2 & 0 & -6 & 0 \\
\hline$\phi_{5}$ & $\mathbf{S}+\mathbf{b}_{2}+\mathbf{b}_{3}$ & $(1,1,1)$ & 0 & -2 & 0 & 0 & 0 & -2 & -2 & $(1,1)$ & -2 & $\overline{0}$ & 6 & 0 \\
\hline$\phi_{6}$ & $+\alpha+2 \gamma$ & $(1,1,1)$ & 0 & 2 & 0 & 0 & 0 & -2 & -2 & $(1,1)$ & 2 & 0 & -6 & 0 \\
\hline$\phi_{7}$ & $\mathbf{S}+\mathbf{b}_{1}+\mathbf{b}_{2}$ & $(1,1,1)$ & 0 & 2 & -2 & 0 & 0 & 0 & 0 & $(1,1)$ & 4 & 0 & 0 & 0 \\
\hline $\bar{\phi}_{7}$ & $+\alpha+\beta$ & $(1,1,1)$ & 0 & -2 & 2 & 0 & 0 & 0 & 0 & $(1,1)$ & -4 & 0 & 0 & 0 \\
\hline$\phi_{8}$ & & $(1,1,1)$ & 0 & 2 & -2 & 0 & 0 & 0 & 0 & $(1,1)$ & -4 & 0 & 0 & 0 \\
\hline $\bar{\phi}_{8}$ & & $(1,1,1)$ & 0 & -2 & 2 & 0 & 0 & 0 & 0 & $(1,1)$ & 4 & 0 & 0 & 0 \\
\hline$\phi_{9}$ & $\mathbf{S}+\mathbf{b}_{1}$ & $(1,1,1)$ & -3 & 3 & 1 & -1 & 0 & 0 & 0 & $(1,1)$ & 2 & -3 & 3 & 0 \\
\hline $\bar{\phi}_{9}$ & $+\mathbf{b}_{3}+\alpha$ & $(1,1,1)$ & 3 & -3 & -1 & 1 & 0 & 0 & 0 & $(1,1)$ & -2 & 3 & -3 & 0 \\
\hline$\phi_{10}$ & $\pm \gamma$ & $(1,1,1)$ & -3 & -1 & -3 & -1 & 0 & 0 & 0 & $(1,1)$ & 2 & -3 & 3 & 0 \\
\hline $\bar{\phi}_{10}$ & & $(1,1,1)$ & 3 & 1 & 3 & 1 & 0 & 0 & 0 & $(1,1)$ & -2 & 3 & -3 & 0 \\
\hline$\phi_{11}$ & & $(1,1,1)$ & -3 & -1 & 1 & 3 & 0 & 0 & 0 & $(1,1)$ & 2 & -3 & 3 & 0 \\
\hline $\bar{\phi}_{11}$ & & $(1,1,1)$ & 3 & 1 & -1 & -3 & 0 & 0 & 0 & $(1,1)$ & -2 & 3 & -3 & 0 \\
\hline$\phi_{12}$ & $\mathbf{S}+\mathbf{b}_{2}$ & $(1,1,1)$ & -3 & -3 & -1 & -1 & 0 & $\overline{0}$ & 0 & $(1,1)$ & -2 & -3 & 3 & 0 \\
\hline $\bar{\Phi}_{31}$ & $+\mathbf{b}_{3}+\beta$ & $(1,1,1)$ & 3 & 3 & 1 & 1 & 0 & 0 & 0 & $(1,1)$ & 2 & 3 & -3 & 0 \\
\hline$\phi_{13}$ & $\pm \gamma$ & $(1,1,1)$ & -3 & 1 & 3 & -1 & 0 & 0 & 0 & $(1,1)$ & -2 & -3 & 3 & 0 \\
\hline $\bar{\phi}_{13}$ & & $(1,1,1)$ & 3 & -1 & -3 & 1 & 0 & 0 & 0 & $(1,1)$ & 2 & 3 & -3 & 0 \\
\hline$\phi_{14}$ & & $(1,1,1)$ & -3 & 1 & -1 & 3 & 0 & 0 & 0 & $(1,1)$ & -2 & -3 & 3 & 0 \\
\hline $\bar{\phi}_{14}$ & & $(1,1,1)$ & 3 & -1 & 1 & -3 & 0 & 0 & 0 & $(1,1)$ & 2 & 3 & -3 & 0 \\
\hline$\phi_{15}$ & $\mathbf{1}+\mathrm{S}+\mathrm{b}_{3}$ & $(1,1,1)$ & 0 & 0 & 0 & -2 & -2 & $\overline{-2}$ & 0 & $(1,1)$ & 0 & 6 & 0 & 2 \\
\hline $\bar{\phi}_{15}$ & $+\alpha+2 \gamma$ & $(1,1,1)$ & 0 & 0 & 0 & 2 & -2 & -2 & 0 & $(1,1)$ & 0 & -6 & 0 & -2 \\
\hline$\phi_{16}$ & $\mathbf{1}+\mathrm{S}+\mathrm{b}_{2}$ & $(1,1,1)$ & 0 & 0 & -2 & 0 & -2 & $\overline{0}$ & -2 & $(1,1)$ & 0 & 6 & 0 & 2 \\
\hline $\bar{\phi}_{16}$ & $+\alpha+2 \gamma$ & $(1,1,1)$ & 0 & 0 & 2 & 0 & -2 & 0 & -2 & $(1,1)$ & 0 & -6 & 0 & -2 \\
\hline$\phi_{17}$ & $\mathbf{1}+\mathbf{S}+\mathbf{b}_{1}$ & $(1,1,1)$ & 0 & -2 & 0 & 0 & 0 & -2 & -2 & $(1,1)$ & 0 & 6 & 0 & 2 \\
\hline $\bar{\phi}_{17}$ & $+\alpha+2 \gamma$ & $(1,1,1)$ & 0 & 2 & 0 & 0 & 0 & -2 & -2 & $(1,1)$ & 0 & -6 & 0 & -2 \\
\hline$\phi_{18}$ & $\mathbf{1}+\mathbf{S}+\mathbf{b}_{3}$ & $(1,1,1)$ & 6 & 0 & 0 & 2 & 0 & $\overline{0}$ & 0 & $(1,1)$ & -2 & 0 & 0 & -2 \\
\hline $\bar{\phi}_{18}$ & $+\alpha+\beta+2 \gamma$ & $(1,1,1)$ & -6 & 0 & 0 & -2 & 0 & 0 & 0 & $(1,1)$ & 2 & 0 & 0 & 2 \\
\hline$\phi_{19}$ & $\mathbf{1}+\mathbf{S}+\mathbf{b}_{1}$ & $(1,1,1)$ & -3 & -3 & -1 & -1 & 0 & $\overline{0}$ & 0 & $(1,1)$ & 0 & 3 & -3 & 2 \\
\hline $\bar{\phi}_{19}$ & $+\beta \pm \gamma$ & $(1,1,1)$ & 3 & 3 & 1 & 1 & 0 & 0 & 0 & $(1,1)$ & 0 & -3 & 3 & -2 \\
\hline$\phi_{20}$ & & $(1,1,1)$ & -3 & 1 & 3 & -1 & 0 & 0 & 0 & $(1,1)$ & 0 & 3 & -3 & 2 \\
\hline $\bar{\phi}_{20}$ & & $(1,1,1)$ & 3 & -1 & -3 & 1 & 0 & 0 & 0 & $(1,1)$ & 0 & -3 & 3 & -2 \\
\hline$\phi_{21}$ & & $(1,1,1)$ & -3 & 1 & -1 & 3 & 0 & 0 & 0 & $(1,1)$ & 0 & 3 & -3 & 2 \\
\hline $\bar{\phi}_{21}$ & & $(1,1,1)$ & 3 & -1 & 1 & -3 & 0 & 0 & 0 & $(1,1)$ & 0 & -3 & 3 & -2 \\
\hline
\end{tabular}

Table 3 continued: Model 3 fields. 


\begin{tabular}{|c|c|c|c|c|c|c|c|c|c|c|c|c|c|c|}
\hline$F$ & SEC & $(C ; L ; R)$ & $Q_{C}$ & $Q_{1}$ & $Q_{2}$ & $Q_{3}$ & $Q_{4}$ & $Q_{5}$ & $Q_{6}$ & $S U(3)_{H_{1 ; 2}}$ & $Q_{7}$ & $Q_{8}$ & $Q_{9}$ & $Q_{10}$ \\
\hline$H_{1}^{+}$ & $\mathbf{1}+\mathbf{S}+\mathbf{b}_{1}$ & $(1,1,1)$ & 0 & 2 & 0 & $\overline{0}$ & 0 & -2 & -2 & $(3,1)$ & 0 & 2 & 0 & -2 \\
\hline$H_{1}^{-}$ & $+\alpha+2 \gamma$ & $(1,1,1)$ & 0 & -2 & 0 & 0 & 0 & -2 & -2 & $(\overline{3}, 1)$ & 0 & -2 & 0 & 2 \\
\hline$H_{2}^{+}$ & $\mathbf{1}+\mathbf{S}+\mathbf{b}_{2}$ & $(1,1,1)$ & 0 & 0 & 2 & 0 & -2 & $\overline{0}$ & -2 & $(3,1)$ & 0 & 2 & 0 & -2 \\
\hline$H_{2}^{-}$ & $+\alpha+2 \gamma$ & $(1,1,1)$ & 0 & 0 & -2 & 0 & -2 & 0 & -2 & $(\overline{3}, 1)$ & 0 & -2 & 0 & 2 \\
\hline$H_{3}^{+}$ & $\mathbf{1}+\mathbf{S}+\mathbf{b}_{3}$ & $(1,1,1)$ & 0 & 0 & 0 & 2 & -2 & -2 & 0 & $(3,1)$ & 0 & 2 & 0 & -2 \\
\hline$H_{3}^{-}$ & $+\alpha+2 \gamma$ & $(1,1,1)$ & 0 & 0 & 0 & -2 & -2 & -2 & 0 & $(\overline{3}, 1)$ & 0 & -2 & 0 & 2 \\
\hline$H_{4}$ & $\mathbf{1}+\mathbf{S}+\mathbf{b}_{1}$ & $(1,1,1)$ & -3 & 1 & -1 & -1 & 0 & 0 & 0 & $(3,1)$ & 0 & -1 & -3 & -2 \\
\hline $\bar{H}_{4}$ & $+\beta \pm \gamma$ & $(1,1,1)$ & 3 & -1 & 1 & 1 & 0 & 0 & 0 & $(\overline{3}, 1)$ & 0 & 1 & 3 & 2 \\
\hline$H_{5}$ & $\mathbf{S}+\mathbf{b}_{1}+\mathbf{b}_{3}$ & $(1,1,1)$ & -3 & -1 & 1 & -1 & 0 & 0 & 0 & $(1,3)$ & -2 & -3 & -1 & 0 \\
\hline $\bar{H}_{5}$ & $+\alpha \pm \gamma$ & $(1,1,1)$ & 3 & 1 & -1 & 1 & 0 & 0 & 0 & $(1, \overline{3})$ & 2 & 3 & 1 & 0 \\
\hline$H_{6}$ & $\mathbf{S}+\mathbf{b}_{2}+\mathbf{b}_{3}$ & $(1,1,1)$ & -3 & 1 & -1 & $\overline{-1}$ & 0 & 0 & 0 & $(1,3)$ & 2 & -3 & -1 & 0 \\
\hline $\bar{H}_{6}$ & $+\beta \pm \gamma$ & $(1,1,1)$ & 3 & -1 & 1 & 1 & 0 & 0 & 0 & $(1, \overline{3})$ & -2 & 3 & 1 & 0 \\
\hline$H_{7}$ & $\mathbf{S}+\mathbf{b}_{1}+\mathbf{b}_{2}$ & $(1,1,1)$ & 0 & 2 & 2 & 0 & 0 & 0 & 0 & $(1,3)$ & 0 & 0 & -4 & 0 \\
\hline $\bar{H}_{7}$ & $+\alpha+\beta$ & $(1,1,1)$ & 0 & -2 & -2 & 0 & 0 & 0 & 0 & $(1, \overline{3})$ & 0 & 0 & 4 & 0 \\
\hline$H_{8}$ & & $(1,1,1)$ & 0 & -2 & -2 & 0 & 0 & 0 & 0 & $(1,3)$ & 0 & 0 & -4 & 0 \\
\hline $\bar{H}_{8}$ & & $(1,1,1)$ & 0 & 2 & 2 & 0 & 0 & 0 & 0 & $(1, \overline{3})$ & 0 & 0 & 4 & 0 \\
\hline$H_{9}$ & $\mathbf{1}+\mathbf{S}+\mathbf{b}_{2}$ & $(1,1,1)$ & 3 & 1 & -1 & 1 & 0 & 0 & 0 & $(1,3)$ & 0 & -3 & -1 & -2 \\
\hline $\bar{H}_{9}$ & $+\alpha \pm \gamma$ & $(1,1,1)$ & -3 & -1 & 1 & -1 & 0 & 0 & 0 & $(1, \overline{3})$ & 0 & 3 & 1 & 2 \\
\hline$H_{10}^{+}$ & $\mathbf{S}+\mathbf{b}_{1}+\mathbf{b}_{2}$ & $(1,1,1)$ & 0 & 0 & 0 & 2 & -2 & -2 & 0 & $(1,3)$ & 2 & 0 & 2 & 0 \\
\hline$H_{10}^{-}$ & $+\alpha+2 \gamma$ & $(1,1,1)$ & 0 & 0 & 0 & -2 & -2 & -2 & 0 & $(1, \overline{3})$ & -2 & 0 & -2 & 0 \\
\hline$\overline{H_{11}^{+}}$ & $\mathbf{S}+\mathbf{b}_{1}+\mathbf{b}_{3}$ & $(1,1,1)$ & 0 & $\overline{0}$ & 2 & $\overline{0}$ & -2 & 0 & -2 & $(1,3)$ & 2 & 0 & 2 & $\overline{0}$ \\
\hline$H_{11}^{-}$ & $+\alpha+2 \gamma$ & $(1,1,1)$ & 0 & 0 & -2 & 0 & -2 & 0 & -2 & $(1, \overline{3})$ & -2 & 0 & -2 & 0 \\
\hline$H_{12}^{+}$ & $\mathbf{S}+\mathbf{b}_{2}+\mathbf{b}_{3}$ & $(1,1,1)$ & 0 & 2 & 0 & $\overline{0}$ & 0 & $\overline{-2}$ & -2 & $(1,3)$ & 2 & 0 & 2 & 0 \\
\hline$H_{12}^{-}$ & $+\alpha+2 \gamma$ & $(1,1,1)$ & 0 & -2 & 0 & 0 & 0 & -2 & -2 & $(1, \overline{3})$ & -2 & 0 & -2 & 0 \\
\hline
\end{tabular}

Table 3 continued: Model 3 fields. 


\section{References}

[1] G.B. Cleaver et al. Phys. Lett. B445 (1999) 135; hep-ph/9904301; hepph/9910230; hep-ph/0002060; hep-ph/0002292.

[2] I. Antoniadis, J. Ellis, J. Hagelin, and D.V. Nanopoulos, Phys. Lett. B231 (1989) 65

I. Antoniadis, J. Ellis, S. Kelley and D.V. Nanopoulos, Phys. Lett. B272 (1991) 31 ;

J.L. Lopez, D.V. Nanopoulos and K. Yuan, Nucl. Phys. B399 (1993) 654.

[3] A.E. Faraggi and D.V. Nanopoulos, Phys. Rev. D48 (1993) 3288.

[4] P. Candelas, G. Horwitz, A. Strominger and E. Witten, Nucl. Phys. B258 (1985) 46 ;

B.R. Greene, K.H. Kirklin, P.J. Miron and G.G. Ross Nucl. Phys. B278 (1986) 667; Nucl. Phys. B292 (1987) 606.

[5] A.E. Faraggi, D.V. Nanopoulos, and K. Yuan, Nucl. Phys. B335 (1990) 347;

A.E. Faraggi, Phys. Rev. D46 (1992) 3204.

[6] I. Antoniadis, G.K. Leontaris and J. Rizos, Phys. Lett. B245 (1990) 161;

G.K. Leontaris and J. Rizos, Nucl. Phys. B554 (1999) 3.

[7] A.E. Faraggi, Nucl. Phys. B387 (1992) 239; hep-ph/9708112.

[8] For a recent review of left-right symmetric models see e.g. R.N. Mohapatra, hep-ph/9912272 and references therein.

[9] M. Pospelov, Phys. Rev. D59 (1997) 259, and references therein.

[10] A. Schellekens, in "Superstring Construction," (North-Holland, New York, 1989) p. 2; A. Schellekens and N. Warner, Nucl. Phys. B313 (89) 41.

[11] T. Kobayashi and H. Nakano, Nucl. Phys. B496 (1997) 103; hep-th/9612066.

[12] G.B. Cleaver and A.E. Faraggi, Int. J. Mod. Phys. A14 (1999) 2335.

[13] R.N. Mohapatra and A. Rasin, Phys. Rev. D54 (1996) 5835;

C.S. Aulakh, A. Melfo, A. Rasin and G. Senjanovic, Phys. Rev. D58 (1998) 115007.

[14] I. Antoniadis, C. Bachas, and C. Kounnas, Nucl. Phys. B289 (1987) 87;

H. Kawai, D.C. Lewellen, and S.H.-H. Tye, Nucl. Phys. B288 (1987) 1.

[15] A.E. Faraggi, Phys. Lett. B278 (1992) 131; Phys. Lett. B274 (1992) 47. 
[16] A.E. Faraggi, Phys. Lett. B339 (1994) 223.

[17] A.E. Faraggi, Nucl. Phys. B428 (1994) 111.

[18] S. Kalara, J.L. Lopez and D.V. Nanopoulos, Nucl. Phys. B353 (1991) 650.

[19] G. Aldazabal, L. Ibanez and F. Quevedo, hep-th/9909172.

[20] I. Antoniadis, J. Ellis, J. Hagelin, and D.V. Nanopoulos, Phys. Lett. B205 (1988) 459 ;

A. Font, L.E. Ibanez, H.P. Nilles and F. Quevedo, Nucl. Phys. B307 (1988) 109; Phys. Lett. B210 (1988) 101;

J.A. Casas and C. Muñoz, Phys. Lett. B209 (1988) 214;

J.A. Casas, E.K. Katehou and C. Muñoz, Nucl. Phys. B317 (1989) 117;

J.L. Lopez and D.V. Nanopoulos, Nucl. Phys. B338 (1990) 73; Phys. Lett. B251 (1990) 73 ;

J. Rizos and K. Tamvakis, Phys. Lett. B251 (1990) 369;

I. Antoniadis, J. Rizos and K. Tamvakis, Phys. Lett. B278 (1992) 257;

A.E. Faraggi, Nucl. Phys. B403 (1993) 101;

G. Cleaver et al. Nucl. Phys. B525 (1998) 3; Nucl. Phys. B545 (1999) 47.

[21] M. Dine, N. Seiberg and E. Witten, Nucl. Phys. B289 (1987) 589;

J. Atick, L. Dixon and A. Sen, Nucl. Phys. B292 (1987) 109.

[22] A.E. Faraggi, Phys. Lett. B329 (1994) 208.

[23] A.E. Faraggi and M. Pospelov, hep-ph/0008223. 